\title{
Quantum Equilibrium and the Role of Operators as Observables in Quantum Theory*
}

\author{
Detlef Dürr \\ Mathematisches Institut der Universität München \\ Theresienstraße 39, 80333 München, Germany \\ E-mail: duerr@mathematik.uni-muenchen.de \\ Sheldon Goldstein \\ Departments of Mathematics, Physics, and Philosophy, Rutgers University \\ 110 Frelinghuysen Road, Piscataway, NJ 08854-8019, USA \\ E-mail: oldstein@math.rutgers.edu \\ Nino Zanghì \\ Dipartimento di Fisica dell'Università di Genova \\ Istituto Nazionale di Fisica Nucleare — Sezione di Genova \\ via Dodecaneso 33, 16146 Genova, Italy \\ E-mail: zanghi@ge.infn.it
}

\begin{abstract}
Bohmian mechanics is the most naively obvious embedding imaginable of Schrödinger's equation into a completely coherent physical theory. It describes a world in which particles move in a highly non-Newtonian sort of way, one which may at first appear to have little to do with the spectrum of predictions of quantum mechanics. It turns out, however, that as a consequence of the defining dynamical equations of Bohmian mechanics, when a system has wave function $\psi$ its configuration is typically random, with probability density $\rho$ given by $|\psi|^{2}$, the quantum equilibrium distribution. It also turns out that the entire quantum formalism, operators as observables and all the rest, naturally emerges in Bohmian mechanics from the analysis of "measurements." This analysis reveals the status of operators as observables in the description of quantum phenomena, and facilitates a clear view of the range of applicability of the usual quantum mechanical formulas.
\end{abstract}

${ }^{*}$ Dedicated to Elliott Lieb on the occasion of his 70th birthday. Elliott will be (we fear unpleasantly) surprised to learn that he bears a greater responsibility for this paper than he could possibly imagine. We would of course like to think that our work addresses in some way the concern suggested by the title of his recent talks, The Quantum-Mechanical World View: A Remarkably Successful but Still Incomplete Theory, but we recognize that our understanding of incompleteness is much more naive than Elliott's. He did, however, encourage us in his capacity as an editor of the Reviews of Modern Physics to submit a paper on the role of operators in quantum theory. That was 12 year ago. Elliott is no longer an editor there and the paper that developed is not quite a review. 


\section{Contents}

\begin{tabular}{lll}
\hline & Introduction & 3 \\
\hline
\end{tabular}

2 Bohmian Experiments $\quad 6$

2.1 Equivariance and Quantum Equilibrium . . . . . . . . . . . . . . . . . . 7

2.2 Conditional and Effective Wave Functions . . . . . . . . . . . . . . . . . . . . . 8

2.3 Decoherence . . . . . . . . . . . . . . . . . . . . . 10

2.4 Wave Function and State . . . . . . . . . . . . . . . . . . . . . . . . . . . . 11

2.5 The Stern-Gerlach Experiment . . . . . . . . . . . . . . . . . . . . 12

2.6 A Remark on the Reality of Spin in Bohmian Mechanics . . . . . . . . . . . . . . 14

2.7 The Framework of Discrete Experiments . . . . . . . . . . . . . . . . . . . . . . . 15

2.8 Reproducibility and its Consequences . . . . . . . . . . . . . . . . . . . . 16

2.9 Operators as Observables . . . . . . . . . . . . . . . . . . . . . 18

2.10 The General Framework of Bohmian Experiments . . . . . . . . . . . . . . . . . . 19

3 The Quantum Formalism 20

3.1 Weak Formal Measurements . . . . . . . . . . . . . . . . . . . . . . . 21

3.2 Strong Formal Measurements . . . . . . . . . . . . . . . . . . . . . . . . 22

3.2.1 Ideal Measurements . . . . . . . . . . . . . . . . . . . . . . . . 22

3.2 .2 Normal Measurements . . . . . . . . . . . . . . . . . . . . . . . 23

3.2.3 Standard Measurements . . . . . . . . . . . . . . . . . . . . 23

3.2.4 Strong Formal Measurements . . . . . . . . . . . . . . . . . . . . . . . . . 24

3.3 From Formal Measurements to Experiments . . . . . . . . . . . . . . . . . . . . . 25

3.4 Von Neumann Measurements . . . . . . . . . . . . . . . . . . . . . . . . 25

3.5 Preparation Procedures . . . . . . . . . . . . . . . . . . . 26

3.6 Measurements of Commuting Families of Operators . . . . . . . . . . . . . . . . 27

3.7 Functions of Measurements . . . . . . . . . . . . . . . . . . . . . 28

3.8 Measurements of Operators with Continuous Spectrum . . . . . . . . . . . . . . . 30

3.9 Sequential Measurements . . . . . . . . . . . . . . . . . . . . . . 30

3.10 Some Summarizing Remarks . . . . . . . . . . . . . . . . . . . . . . . 32

4 The Extended Quantum Formalism

4.1 POVMs and Bohmian Experiments . . . . . . . . . . . . . . . . . . . . 34

4.2 Formal Experiments . . . . . . . . . . . . . . . . . . . 35

4.3 From Formal Experiments to Experiments . . . . . . . . . . . . . . . . . . . . 38

4.4 Measure-Valued Quadratic Maps . . . . . . . . . . . . . . . . . . . . . . . . . 39

5 The General Emergence of Operators $\quad 40$

5.1 "No Interaction" Experiments . . . . . . . . . . . . . . . . . . . . . . . . . . 41

5.2 "No $X$ " Experiments . . . . . . . . . . . . . . . . . . . . . . . . . 41

5.3 "No $Y$ " Experiments . . . . . . . . . . . . . . . . . . . . . . . . . . . . . . . . 43

5.4 "No $Y$ no $\Phi "$ Experiments . . . . . . . . . . . . . . . . . . . . . . . . 44

5.5 The Basic Operators of Ouantum Mechanics . . . . . . . . . . . . . . . . . . . . . 44

5.6 From Positive-Operator-Valued Measures to Experiments . . . . . . . . . . . . . . 46

5.7 Invariance Under Trivial Extension . . . . . . . . . . . . . . . . . . . . . . . . . 47 
5.8 POVMs and the Positions of Photons and Dirac Electrons . . . . . . . . . . . . 47

6 Density Matrices 48

6.1 Densitv Matrices and Bohmian Experiments . . . . . . . . . . . . . . . . . . . 49

6.2 Strong Experiments and Density Matrices . . . . . . . . . . . . . . . . 50

6.3 The Notion of Instrument . . . . . . . . . . . . . . . . . . . . . . . . 51

6.4 On the State Description Provided by Density Matrices . . . . . . . . . . . . . . . 51

$\begin{array}{lll}7 & \text { Genuine Measurements } & 53\end{array}$

7.1 A Necessarv Condition for Measurabilitv . . . . . . . . . . . . . . . . . 54

7.2 The Nonmeasurabilitv of Velocity. Wave Function and Deterministic Quantities . 55

7.3 Initial Values and Final Values . . . . . . . . . . . . . . . . . . . . 55

7.4 Nonlinear Measurements and the Role of Prior Information . . . . . . . . . . . 56

7.5 A Position Measurement that Does not Measure Position . . . . . . . . . . . . . . 57

7.6 Theorv Dependence of Measurement . . . . . . . . . . . . . . . . . 58

8 Hidden Variables $\quad 59$

8.1 Experiments and Random Variables . . . . . . . . . . . . . . . . . . . . . 59

8.2 Random Variables. Operators, and the Impossibility Theorems . . . . . . . . . . . 60

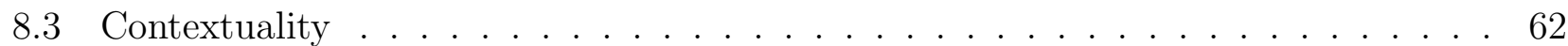

8.4 Against "Contextualitv" . . . . . . . . . . . . . . . . . . . . . 64

8.5 Nonlocality Contextualitv and Hidden Variables . . . . . . . . . . . . . . . 67

9 Against Naive Realism About Operators $\quad 70$

\begin{tabular}{ll}
\hline Acknowledgments & 72
\end{tabular}

\begin{tabular}{ll}
\hline References & 72
\end{tabular}

\section{Introduction}

It is often argued that the quantum mechanical association of observables with self-adjoint operators is a straightforward generalization of the notion of classical observable, and that quantum theory should be no more conceptually problematic than classical physics once this is appreciated. The classical physical observables - for a system of particles, their positions $q=\left(\mathbf{q}_{k}\right)$, their momenta $p=\left(\mathbf{p}_{k}\right)$, and the functions thereof, i.e., functions on phase space - form a commutative algebra. It is generally taken to be the essence of quantization, the procedure which converts a classical theory to a quantum one, that $q, p$, and hence all functions $f(q, p)$ thereof are replaced by appropriate operators, on a Hilbert space (of possible wave functions) associated with the system under consideration. Thus quantization leads to a noncommutative operator algebra of "observables," the standard examples of which are provided by matrices and linear operators. Thus it seems perfectly natural that classical observables are functions on phase space and quantum observables are self-adjoint operators.

However, there is much less here than meets the eye. What should be meant by "measuring" a quantum observable, a self-adjoint operator? We think it is clear that this must be specifiedwithout such specification it can have no meaning whatsoever. Thus we should be careful here 
and use safer terminology by saying that in quantum theory observables are associated with selfadjoint operators, since it is difficult to see what could be meant by more than an association, by an identification of observables, regarded as somehow having independent meaning relating to observation or measurement (if not to intrinsic "properties"), with such a mathematical abstraction as a self-adjoint operator.

We are insisting on "association" rather than identification in quantum theory, but not in classical theory, because there we begin with a rather clear notion of observable (or property) which is well-captured by the notion of a function on the phase space, the state space of complete descriptions. If the state of the system were observed, the value of the observable would of course be given by this function of the state $(q, p)$, but the observable might be observed by itself, yielding only a partial specification of the state. In other words, measuring a classical observable means determining to which level surface of the corresponding function the state of the system, the phase point - which is at any time definite though probably unknown - belongs. In the quantum realm the analogous notion could be that of function on Hilbert space, not selfadjoint operator. But we don't measure the wave function, so that functions on Hilbert space are not physically measurable, and thus do not define "observables."

The problematical character of the way in which measurement is treated in orthodox quantum theory has been stressed by John Bell:

The concept of 'measurement' becomes so fuzzy on reflection that it is quite surprising to have it appearing in physical theory at the most fundamental level. Less surprising perhaps is that mathematicians, who need only simple axioms about otherwise undefined objects, have been able to write extensive works on quantum measurement theory - which experimental physicists do not find it necessary to read. ... Does not any analysis of measurement require concepts more fundamental than measurement? And should not the fundamental theory be about these more fundamental concepts? 8]

... in physics the only observations we must consider are position observations, if only the positions of instrument pointers. It is a great merit of the de Broglie-Bohm picture to force us to consider this fact. If you make axioms, rather than definitions and theorems, about the 'measurement' of anything else, then you commit redundancy and risk inconsistency. 9]

The Broglie-Bohm theory, Bohmian mechanics, is a physical theory for which the concept of 'measurement' does not appear at the most fundamental level - in the very formulation of the theory. It is a theory about concepts more fundamental than 'measurement,' in terms of which an analysis of measurement can be performed. In a previous work [25] we have shown how probabilities for positions of particles given by $|\psi|^{2}$ emerge naturally from an analysis of "equilibrium" for the deterministic dynamical system defined by Bohmian mechanics, in much the same way that the Maxwellian velocity distribution emerges from an analysis of classical thermodynamic equilibrium. Our analysis entails that Born's statistical rule $\rho=|\psi|^{2} \mid$ should be regarded as a local manifestation of a global equilibrium state of the universe, what we call quantum equilibrium, a concept analogous to, but quite distinct from, thermodynamic equilibrium: a universe in quantum equilibrium evolves so as to yield an appearance of randomness, with empirical distributions in agreement with all the predictions of the quantum formalism.

While in our earlier work we have proven, from the first principles of Bohmian mechanics, the "quantum equilibrium hypothesis" that when a system has wave function $\psi$, the distribution 
$\rho$ of its configuration satisfies $\rho=|\psi|^{2}$, our goal here is to show that it follows from this hypothesis, not merely that Bohmian mechanics makes the same predictions as does orthodox quantum theory for the results of any experiment, but that the quantum formalism of operators as observables emerges naturally and simply as the very expression of the empirical import of Bohmian mechanics.

More precisely, we shall show here that self-adjoint operators arise in association with specific experiments: insofar as the statistics for the values which result from the experiment are concerned, the notion of self-adjoint operator compactly expresses and represents the relevant data. It is the association " $\mathscr{E} \mapsto A$ " between an experiment $\mathscr{E}$ and an operator $A$ - an association that we shall establish in Section 2 and upon which we shall elaborate in the other sections - that is the central notion of this paper. According to this association the notion of operator-as-observable in no way implies that anything is measured in the experiment, and certainly not the operator itself. We shall nonetheless speak of such experiments as measurements, since this terminology is unfortunately standard. When we wish to emphasize that we really mean measurement - the ascertaining of the value of a quantity - we shall often speak of genuine measurement.

Much of our analysis of the emergence and role of operators as observables in Bohmian mechanics, including the von Neumann-type picture of measurements at which we shall arrive, applies as well to orthodox quantum theory. Indeed, the best way to understand the status of the quantum formalism - and to better appreciate the minimality of Bohmian mechanics - is Bohr's way: What are called quantum observables obtain meaning only through their association with specific experiments. We believe that Bohr's point has not been taken to heart by most physicists, even those who regard themselves as advocates of the Copenhagen interpretation.

Indeed, it would appear that the argument provided by our analysis against taking operators too seriously as observables has even greater force from an orthodox perspective: Given the initial wave function, at least in Bohmian mechanics the outcome of the particular experiment is determined by the initial configuration of system and apparatus, while for orthodox quantum theory there is nothing in the initial state which completely determines the outcome. Indeed, we find it rather surprising that most proponents of standard quantum measurement theory, that is the von Neumann analysis of measurement [74, beginning with von Neumann, nonetheless seem to retain an uncritical identification of operators with properties. Of course, this is presumably because more urgent matters - the measurement problem and the suggestion of inconsistency and incoherence that it entails - soon force themselves upon one's attention. Moreover such difficulties perhaps make it difficult to maintain much confidence about just what should be concluded from the "measurement" analysis, while in Bohmian mechanics, for which no such difficulties arise, what should be concluded is rather obvious.

Moreover, a great many significant real-world experiments are simply not at all associated with operators in the usual way. Because of these and other difficulties, it has been proposed that we should go beyond operators-as-observables, to generalized observables, described by mathematical objects (positive-operator-valued measures, POVMs) even more abstract than operators (see, e.g., the books of Davies [21, Holevo [50] and Kraus [55]). It may seem that we would regard this development as a step in the wrong direction, since it supplies us with a new, much larger class of abstract mathematical entities about which to be naive realists. We shall, however, show that these generalized observables for Bohmian mechanics form an extremely natural class of objects to associate with experiments, and that the emergence and role these observables is merely an expression of quantum equilibrium together with the linearity of Schrödinger's evolution. It is 
therefore rather dubious that the occurrence of generalized observables - the simplest case of which are self-adjoint operators - can be regarded as suggesting any deep truths about reality or about epistemology.

As a byproduct of our analysis of measurement we shall obtain a criterion of measurability and use it to examine the genuine measurability of some of the properties of a physical system. In this regard, it should be stressed that measurability is theory-dependent: different theories, though empirically equivalent, may differ on what should be regarded as genuinely measurable within each theory. This important - though very often ignored - point was made long ago by Einstein and has been repeatedly stressed by Bell. It is best summarized by Einstein's remark [49]: "It is the theory which decides what we can observe."

We note in passing that measurability and reality are different issues. Indeed, for Bohmian mechanics most of what is "measurable" (in a sense that we will explain) is not real and most of what is real is not genuinely measurable. (The main exception, the position of a particle, which is both real and genuinely measurable, is, however, constrained by absolute uncertainty [25]).

In focusing here on the role of operators as observables, we don't wish to suggest that there are no other important roles played by operators in quantum theory. In particular, in addition to the familiar role played by operators as generators of symmetries and time-evolutions, we would like to mention the rather different role played by the field operators of quantum field theory: to link abstract Hilbert-space to space-time and structures therein, facilitating the formulation of theories describing the behavior of an indefinite number of particles [30, 29].

Finally, we should mention what should be the most interesting sense of measurement for a physicist, namely the determination of the coupling constants and other parameters that define our physical theories. This has little to do with operators as observables in quantum theory and shall not be addressed here.

\section{Notations and Conventions}

$Q=\left(\mathbf{Q}_{1}, \ldots, \mathbf{Q}_{N}\right)$ denotes the actual configuration of a system of $N$ particle with positions $\mathbf{Q}_{k} ; q=\left(\mathbf{q}_{1}, \ldots, \mathbf{q}_{N}\right)$ is its generic configuration. Whenever we deal with a system-apparatus composite, $x(X)$ will denote the generic (actual) configuration of the system and $y(Y)$ that of the apparatus. Sometimes we shall refer to the system as the $x$-system and the apparatus

as the $y$-system. Since the apparatus should be understood as including all systems relevant to the behavior of the system in which we are interested, this notation and terminology is quite compatible with that of Section [2.2, in which $y$ refers to the environment of the $x$-system.

For a system in the state $\Psi, \rho_{\Psi}$ will denote the quantum equilibrium measure, $\rho_{\Psi}(d q)=$ $|\Psi(q)|^{2} d q$. If $Z=F(Q)$ then $\rho_{\Psi}^{Z}$ denotes the measure induced by $F$, i.e. $\rho_{\Psi}^{Z}=\rho_{\Psi} \circ F^{-1}$.

\section{Bohmian Experiments}

According to Bohmian mechanics, the complete description or state of an $N$-particle system is provided by its wave function $\Psi(q, t)$, where $q=\left(\mathbf{q}_{1}, \ldots, \mathbf{q}_{N}\right) \in \mathbb{R}^{3 N}$, and its configuration $Q=\left(\mathbf{Q}_{1}, \ldots, \mathbf{Q}_{N}\right) \in \mathbb{R}^{3 N}$, where the $\mathbf{Q}_{k}$ are the positions of the particles. The wave function, which evolves according to Schrödinger's equation,

$$
i \hbar \frac{\partial \Psi}{\partial t}=H \Psi
$$


choreographs the motion of the particles: these evolve according to the equation

$$
\frac{d \mathbf{Q}_{k}}{d t}=\frac{\hbar}{m_{k}} \operatorname{Im} \frac{\Psi^{*} \nabla_{k} \Psi}{\Psi^{*} \Psi}\left(\mathbf{Q}_{1}, \ldots, \mathbf{Q}_{N}\right)
$$

where $\boldsymbol{\nabla}_{k}=\partial / \partial \mathbf{q}_{k}$. In equation (2.1),$H$ is the usual nonrelativistic Schrödinger Hamiltonian; for spinless particles it is of the form

$$
H=-\sum_{k=1}^{N} \frac{\hbar^{2}}{2 m_{k}} \nabla_{k}^{2}+V
$$

containing as parameters the masses $m_{1} \ldots, m_{N}$ of the particles as well as the potential energy function $V$ of the system. For an $N$-particle system of nonrelativistic particles, equations (2.1) and (2.2) form a complete specification of the theory (magnetic fields ${ }^{1}$ and spin, ${ }^{2}$ as well as Fermi and Bose-Einstein statistics, ${ }^{3}$ can easily be dealt with and in fact arise in a natural manner 6. 15, 64, 42, 28]). There is no need, and indeed no room, for any further axioms, describing either the behavior of other observables or the effects of measurement.

\subsection{Equivariance and Quantum Equilibrium}

It is important to bear in mind that regardless of which observable one chooses to measure, the result of the measurement can be assumed to be given configurationally, say by some pointer orientation or by a pattern of ink marks on a piece of paper. Then the fact that Bohmian mechanics makes the same predictions as does orthodox quantum theory for the results of any experiment-for example, a measurement of momentum or of a spin component-provided we assume a random distribution for the configuration of the system and apparatus at the beginning of the experiment given by $|\Psi(q)|^{2}$ - is a more or less immediate consequence of (2.2). This is because of the quantum continuity equation

$$
\frac{\partial|\Psi|^{2}}{\partial t}+\operatorname{div} J^{\Psi}=0
$$

which is a simple consequence of Schrödinger's equation. Here $J^{\Psi}=\left(\mathbf{J}_{1}^{\Psi}, \ldots, \mathbf{J}_{N}^{\Psi}\right)$ with

$$
\mathbf{J}_{k}^{\Psi}=\frac{\hbar}{m_{k}} \operatorname{Im}\left(\Psi^{*} \nabla_{k} \Psi\right)
$$

the quantum probability current. This equation becomes the classical continuity equation

$$
\frac{\partial \rho}{\partial t}+\operatorname{div} \rho v=0
$$

for the system of equations $d Q / d t=v$ defined by (2.2) - governing the evolution of the probability density $\rho$ under the motion defined by the guiding equation (2.2) for the particular choice $\rho=$

\footnotetext{
${ }^{1}$ When a magnetic field is present, the gradients $\nabla_{k}$ in the equations (2.1) and (2.2) must be understood as the covariant derivatives involving the vector potential $\boldsymbol{A}$.

${ }^{2}$ See Section 2.5

${ }^{3}$ For indistinguishable particles, a careful analysis [28] of the natural configuration space, which is no longer $\mathbb{R}^{3 N}$, leads to the consideration of wave functions on $\mathbb{R}^{3 N}$ that are either symmetric or antisymmetric under permutations.
} 
$|\Psi|^{2}=\Psi^{*} \Psi$. In other words, if the probability density for the configuration satisfies $\rho\left(q, t_{0}\right)=$ $\left|\Psi\left(q, t_{0}\right)\right|^{2}$ at some time $t_{0}$, then the density to which this is carried by the motion (2.2) at any time $t$ is also given by $\rho(q, t)=|\Psi(q, t)|^{2}$. This is an extremely important property of any Bohmian system, as it expresses a certain compatibility between the two equations of motion defining the dynamics, which we call the equivariance ${ }^{4}$ of $|\Psi|^{2}$.

The above assumption guaranteeing agreement between Bohmian mechanics and quantum mechanics regarding the results of any experiment is what we call the "quantum equilibrium hypothesis":

When a system has wave function $\Psi$ its configuration $Q$ is random with probability distribution given by the measure $\rho_{\Psi}(d q)=|\Psi(q)|^{2} d q$.

When this condition is satisfied we shall say that the system is in quantum equilibrium and we shall call $\rho_{\Psi}$ the quantum equilibrium distribution. While the meaning and justification of (2.5) is a delicate matter, which we have discussed at length elsewhere [25], it is important to recognize that, merely as a consequence of (2.2) and (2.5), Bohmian mechanics is a counterexample to all of the claims to the effect that a deterministic theory cannot account for quantum randomness in the familiar statistical mechanical way, as arising from averaging over ignorance: Bohmian mechanics is clearly a deterministic theory, and, as we have just explained, it does account for quantum randomness as arising from averaging over ignorance given by $|\Psi(q)|^{2}$.

\subsection{Conditional and Effective Wave Functions}

Which systems should be governed by Bohmian mechanics? An $n$-particle subsystem of an $N$ particle system $(n<N)$ need not in general be governed by Bohmian mechanics, since no wave function for the subsystem need exist. This will be so even with trivial interaction potential $V$, if the wave function of the system does not properly factorize; for nontrivial $V$ the Schrödinger evolution would in any case quickly destroy such a factorization. Therefore in a universe governed by Bohmian mechanics there is a priori only one wave function, namely that of the universe, and there is a priori only one system governed by Bohmian mechanics, namely the universe itself.

Consider then an $N$-particle non relativistic universe governed by Bohmian mechanics, with (universal) wave function $\Psi$. Focus on a subsystem with configuration variables $x$, i.e., on a splitting $q=(x, y)$ where $y$ represents the configuration of the environment of the $x$-system. The actual particle configurations at time $t$ are accordingly denoted by $X_{t}$ and $Y_{t}$, i.e., $Q_{t}=\left(X_{t}, Y_{t}\right)$. Note that $\Psi_{t}=\Psi_{t}(x, y)$. How can one assign a wave function to the $x$-system? One obvious possibility - afforded by the existence of the actual configuration - is given by what we call the

${ }^{4}$ Equivariance can be formulated in very general terms: consider the transformations $U: \Psi \rightarrow U \Psi$ and $f: Q \rightarrow f(Q)$, where $U$ is a unitary transformation on $L^{2}(d q)$ and $f$ is a transformation on configuration space that may depend on $\Psi$. We say that the map $\Psi \mapsto \mu_{\Psi}$ from wave functions to measures on configuration space is equivariant with respect to $U$ and $f$ if $\mu_{U \Psi}=\mu_{\Psi} \circ f^{-1}$. The above argument based on the continuity equation (2.4) shows that $\Psi \mapsto|\Psi|^{2} d q$ is equivariant with respect to $U \equiv U_{t}=e^{-i \frac{t}{\hbar} H}$, where $H$ is the Schrödinger Hamiltonian (2.3) and $f \equiv f_{t}$ is the solution map of (2.2). In this regard, it is important to observe that for a Hamiltonian $H$ which is not of Schrödinger type we shouldn't expect (2.2) to be the appropriate velocity field, that is, a field which generates an evolution in configuration space having $|\Psi|^{2}$ as equivariant density. For example, for $H=c \frac{\hbar}{i} \frac{\partial}{\partial q}$, where $c$ is a constant (for simplicity we are assuming configuration space to be one-dimensional), we have that $|\Psi|^{2}$ is equivariant provided the evolution of configurations is given by $d Q / d t=c$. In other words, for $U_{t}=e^{c t \frac{\partial}{\partial q}}$ the map $\Psi \mapsto|\Psi|^{2} d q$ is equivariant if $f_{t}: Q \rightarrow Q+c t$. 
conditional wave function of the $x$-system

$$
\psi_{t}(x)=\Psi_{t}\left(x, Y_{t}\right)
$$

To get familiar with this notion consider a very simple one dimensional universe made of two particles with Hamiltonian $(\hbar=1)$

$$
H=H^{(x)}+H^{(y)}+H^{(x y)}=-\frac{1}{2}\left(\frac{\partial^{2}}{\partial x^{2}}+\frac{\partial^{2}}{\partial y^{2}}\right)+\frac{1}{2}(x-y)^{2} .
$$

and initial wave function

$$
\Psi_{0}=\psi \otimes \Phi_{0} \quad \text { with } \quad \psi(x)=\pi^{-\frac{1}{4}} e^{-\frac{x^{2}}{2}} \quad \text { and } \quad \Phi_{0}(y)=\pi^{-\frac{1}{4}} e^{-\frac{y^{2}}{2}} .
$$

Then (2.1) and (2.2) are easily solved:

$$
\begin{gathered}
\Psi_{t}(x, y)=\pi^{-\frac{1}{2}}(1+i t)^{-\frac{1}{2}} e^{-\frac{1}{4}\left[(x-y)^{2}+\frac{(x+y)^{2}}{1+2 i t}\right]}, \\
X_{t}=a(t) X+b(t) Y \text { and } Y_{t}=b(t) X+a(t) Y,
\end{gathered}
$$

where $a(t)=\frac{1}{2}\left[\left(1+t^{2}\right)^{\frac{1}{2}}+1\right], b(t)=\frac{1}{2}\left[\left(1+t^{2}\right)^{\frac{1}{2}}-1\right]$, and $X$ and $Y$ are the initial positions of the two particles. Focus now on one of the two particles (the $x$-system) and regard the other one as its environment (the $y$-system). The conditional wave function of the $x$-system

$$
\psi_{t}(x)=\pi^{-\frac{1}{2}}(1+i t)^{-\frac{1}{2}} e^{-\frac{1}{4}\left[\left(x-Y_{t}\right)^{2}+\frac{\left(x+Y_{t}\right)^{2}}{1+2 i t}\right]},
$$

depends, through $Y_{t}$, on both the initial condition $Y$ for the environment and the initial condition $X$ for the particle. As these are random, so is the evolution of $\psi_{t}$, with probability law determined by $\left|\Psi_{0}\right|^{2}$. In particular, $\psi_{t}$ does not satisfy Schrödinger's equation for any $H^{(x)}$.

We remark that even when the $x$-system is dynamically decoupled from its environment, its conditional wave function will not in general evolve according to Schrödinger's equation. Thus the conditional wave function lacks the dynamical implications from which the wave function of a system derives much of its physical significance. These are, however, captured by the notion of effective wave function:

Suppose that $\Psi(x, y)=\psi(x) \Phi(y)+\Psi^{\perp}(x, y)$, where $\Phi$ and $\Psi^{\perp}$ have macroscopically disjoint $y$-supports. If $Y \in \operatorname{supp} \Phi$ we say that $\psi$ is the effective wave function of the x-system.

Of course, $\psi$ is also the conditional wave function since nonvanishing scalar multiples of wave functions are naturally identified. ${ }^{5}$

\footnotetext{
${ }^{5}$ Note that in Bohmian mechanics the wave function is naturally a projective object since wave functions differing by a multiplicative constant-possibly time-dependent - are associated with the same vector field, and thus generate the same dynamics.
} 


\subsection{Decoherence}

One might wonder why systems possess an effective wave function at all. In fact, in general they don't! For example the $x$-system will not have an effective wave function when, for instance, it belongs to a larger microscopic system whose effective wave function doesn't factorize in the appropriate way. However, the larger the environment of the $x$-system, the greater is the potential for the existence of an effective wave function for this system, owing in effect to the abundance of "measurement-like" interaction with a larger environment. ${ }^{6}$

We remark that it is the relative stability of the macroscopic disjointness employed in the definition of the effective wave function, arising from what are nowadays often called mechanisms of decoherence - the destruction of the coherent spreading of the wave function, the effectively irreversible flow of "phase information" into the (macroscopic) environment-which accounts for the fact that the effective wave function of a system obeys Schrödinger's equation for the system alone whenever this system is isolated. One of the best descriptions of the mechanisms of decoherence, though not the word itself, can be found in Bohm's 1952 "hidden variables" paper 15.

Decoherence plays a crucial role in the very formulation of the various interpretations of quantum theory loosely called decoherence theories(Griffiths [46], Omnès [65], Leggett 60], Zurek [79, Joos and Zeh [51, Gell-Mann and Hartle [35]). In this regard we wish to emphasize, however, as did Bell in his article "Against Measurement" [11, that decoherence in no way comes to grips with the measurement problem itself, being arguably a necessary, but certainly not a sufficient, condition for its complete resolution. In contrast, for Bohmian mechanics decoherence is purely phenomenological - it plays no role whatsoever in the formulation (or interpretation) of the theory itself $^{7}$ — and the very notion of effective wave function accounts at once for the reduction of the wave packet in quantum measurement.

According to orthodox quantum measurement theory [74, 14, 76, 77, after a measurement, or preparation, has been performed on a quantum system, the $x$-system, the wave function for the composite formed by system and apparatus is of the form

$$
\sum_{\alpha} \psi_{\alpha} \otimes \Phi_{\alpha}
$$

with the different $\Phi_{\alpha}$ supported by the macroscopically distinct (sets of) configurations corresponding to the various possible outcomes of the measurement, e.g., given by apparatus pointer orientations. Of course, for Bohmian mechanics the terms of (2.8) are not all on the same footing: one of them, and only one, is selected, or more precisely supported, by the outcome corresponding, say, to $\alpha_{0}$-which actually occurs. To emphasize this we may write (2.8) in the

\footnotetext{
${ }^{6}$ To understand how this comes about one may suppose that initially the $y$-supports of $\Phi$ and $\Psi^{\perp}$ (cf. the definition above of effective wave function) are just "sufficiently" (but not macroscopically) disjoint. Then, due to the interaction with the environment, the amount of $y$-disjointness will tend to increase dramatically as time goes on, with, as in a chain reaction, more and more degrees of freedom participating in this disjointness. When the effect of this "decoherence" is taken into account, one finds that even a small amount of $y$-disjointness will often tend to become "sufficient," and quickly "more than sufficient," and finally macroscopic.

${ }^{7}$ However, decoherence plays an important role in the emergence of Newtonian mechanics as the description of the macroscopic regime for Bohmian mechanics, supporting a picture of a macroscopic Bohmian particle, in the classical regime, guided by a macroscopically well-localized wave packet with a macroscopically sharp momentum moving along a classical trajectory. It may, indeed, seem somewhat ironic that the gross features of our world should appear classical because of interaction with the environment and the resulting wave function entanglement, the characteristic quantum innovation.
} 
form

$$
\psi \otimes \Phi+\Psi^{\perp}
$$

where $\psi=\psi_{\alpha_{0}}, \Phi=\Phi_{\alpha_{0}}$, and $\Psi^{\perp}=\sum_{\alpha \neq \alpha_{0}} \psi_{\alpha} \otimes \Phi_{\alpha}$. By comparison with (2.7) it follows that after the measurement the $x$-system has effective wave function $\psi_{\alpha_{0}}$. This is how collapse (or reduction) of the effective wave function to the one associated with the outcome $\alpha_{0}$ arises in Bohmian mechanics.

While in orthodox quantum theory the "collapse" is merely superimposed upon the unitary evolution - without a precise specification of the circumstances under which it may legitimately be invoked - we have now, in Bohmian mechanics, that the evolution of the effective wave function is actually given by a stochastic process, which consistently embodies both unitarity and collapse as appropriate. In particular, the effective wave function of a subsystem evolves according to Schrödinger's equation when this system is suitably isolated. Otherwise it "pops in and out" of existence in a random fashion, in a way determined by the continuous (but still random) evolution of the conditional wave function $\psi_{t}$. Moreover, it is the critical dependence on the state of the environment and the initial conditions which is responsible for the random behavior of the (conditional or effective) wave function of the system.

\subsection{Wave Function and State}

As an important consequence of (2.6) we have, for the conditional probability distribution of the configuration $X_{t}$ of a system at time $t$, given the configuration $Y_{t}$ of its environment, the fundamental conditional probability formula [25]:

$$
\operatorname{Prob}_{\Psi_{0}}\left(X_{t} \in d x \mid Y_{t}\right)=\left|\psi_{t}(x)\right|^{2} d x
$$

where

$$
\operatorname{Prob}_{\Psi_{0}}(d Q)=\left|\Psi_{0}(Q)\right|^{2} d Q
$$

with $Q=(X, Y)$ the configuration of the universe at the (initial) time $t=0$. Formula (2.9) is the cornerstone of our analysis [25] on the origin of randomness in Bohmian mechanics. Since the right hand side of (2.9) involves only the effective wave function, it follows that the wave function $\psi_{t}$ of a subsystem represents maximal information about its configuration $X_{t}$. In other words, given the fact that its wave function is $\psi_{t}$, it is in principle impossible to know more about the configuration of a system than what is expressed by the right hand side of (2.9), even when the detailed configuration $Y_{t}$ of its environment is taken into account 25]

$$
\operatorname{Prob}_{\Psi_{0}}\left(X_{t} \in d x \mid Y_{t}\right)=\operatorname{Prob}_{\Psi_{0}}\left(X_{t} \in d x \mid \psi_{t}\right)=\left|\psi_{t}(x)\right|^{2} d x .
$$

The fact that the knowledge of the configuration of a system must be mediated by its wave function may partially account for the possibility of identifying the state of a system-its complete description - with its wave function without encountering any practical difficulties. This is primarily because of the wave function's statistical role, but its dynamical role is also relevant here. Thus it is natural, even in Bohmian mechanics, to regard the wave function as the "state" of the system. This attitude is supported by the asymmetric roles of configuration and wave function: while the fact that the wave function is $\psi$ entails that the configuration is distributed according to $|\psi|^{2}$, the fact that the configuration is $X$ has no implications whatsoever for the 
wave function. ${ }^{8}$ Indeed, such an asymmetry is grounded in the dynamical laws and in the initial conditions. $\psi$ is always assumed to be fixed, being usually under experimental control, while $X$ is always taken as random, according to the quantum equilibrium distribution.

When all is said and done, it is important to bear in mind that regarding $\psi$ as the "state" is only of practical value, and shouldn't obscure the more important fact that the most detailed description - the complete state description - is given (in Bohmian mechanics) by the wave function and the configuration.

\subsection{The Stern-Gerlach Experiment}

Information about a system does not spontaneously pop into our heads or into our (other) "measuring" instruments; rather, it is generated by an experiment: some physical interaction between the system of interest and these instruments, which together (if there is more than one) comprise the apparatus for the experiment. Moreover, this interaction is defined by, and must be analyzed in terms of, the physical theory governing the behavior of the composite formed by system and apparatus. If the apparatus is well designed, the experiment should somehow convey significant information about the system. However, we cannot hope to understand the significance of this "information" - for example, the nature of what it is, if anything, that has been measured - without some such theoretical analysis.

As an illustration of such an analysis we shall discuss the Stern-Gerlach experiment from the standpoint of Bohmian mechanics. But first we must explain how spin is incorporated into Bohmian mechanics: If $\Psi$ is spinor-valued, the bilinear forms appearing in the numerator and denominator of (2.2) should be understood as spinor-inner-products; e.g., for a single spin $\frac{1}{2}$ particle the two-component wave function

$$
\Psi \equiv\left(\begin{array}{c}
\Psi_{+}(\mathbf{x}) \\
\Psi_{-}(\mathbf{x})
\end{array}\right)
$$

generates the velocity

$$
\mathbf{v}^{\Psi}=\frac{\hbar}{m} \operatorname{Im} \frac{(\Psi, \nabla \Psi)}{(\Psi, \Psi)}
$$

where $(\cdot, \cdot)$ denotes the scalar product in the spin space $\mathbb{C}^{2}$. The wave function evolves via (2.1), where now the Hamiltonian $H$ contains the Pauli term, for a single particle proportional to $\mathbf{B} \cdot \boldsymbol{\sigma}$, that represents the coupling between the "spin" and an external magnetic field $\mathbf{B}$; here $\boldsymbol{\sigma}=\left(\sigma_{x}, \sigma_{y}, \sigma_{z}\right)$ are the Pauli spin matrices which can be taken to be

$$
\sigma_{x}=\left(\begin{array}{cc}
0 & 1 \\
1 & 0
\end{array}\right) \quad \sigma_{y}=\left(\begin{array}{cc}
0 & -i \\
i & 0
\end{array}\right) \quad \sigma_{z}=\left(\begin{array}{cc}
1 & 0 \\
0 & -1
\end{array}\right)
$$

Let's now focus on a Stern-Gerlach "measurement of the operator $\sigma_{z}$ ": An inhomogeneous magnetic field $\mathbf{B}$ is established in a neighborhood of the origin, by means of a suitable arrangement of magnets. This magnetic field is oriented in the positive $z$-direction, and is increasing in this direction. We also assume that the arrangement is invariant under translations in the $x$-direction, i.e., that the geometry does not depend upon $x$-coordinate. A particle with a fairly definite

\footnotetext{
${ }^{8}$ The "fact" (that the configuration is $X$ ) shouldn't be confused with the "knowledge of the fact": the latter does have such implications [25]!
} 
momentum is directed towards the origin along the negative $y$-axis. For simplicity, we shall consider a neutral spin-1/2 particle whose wave function $\Psi$ evolves according to the Hamiltonian

$$
H=-\frac{\hbar^{2}}{2 m} \nabla^{2}-\mu \boldsymbol{\sigma} \cdot \mathbf{B}
$$

where $\mu$ is a positive constant (if one wishes, one might think of a fictitious electron not feeling the Lorentz force).

The inhomogeneous field generates a vertical deflection of $\Psi$ away from the $y$-axis, which for Bohmian mechanics leads to a similar deflection of the particle trajectory according to the velocity field defined by (2.11): if its wave function $\Psi$ were initially an eigenstate of $\sigma_{z}$ of eigenvalue 1 or -1 , i.e., if it were of the form

$$
\Psi^{(+)}=\psi^{(+)} \otimes \Phi_{0}(\mathbf{x}) \quad \text { or } \quad \Psi^{(-)}=\psi^{(-)} \otimes \Phi_{0}(\mathbf{x})
$$

where

$$
\psi^{(+)} \equiv\left(\begin{array}{l}
1 \\
0
\end{array}\right) \quad \text { and } \quad \psi^{(-)} \equiv\left(\begin{array}{l}
0 \\
1
\end{array}\right)
$$

then the deflection would be in the positive (negative) $z$-direction (by a rather definite angle). This limiting behavior is readily seen for $\Phi_{0}=\Phi_{0}(z) \phi(x, y)$ and $\mathbf{B}=(0,0, B)$, so that the $z$ motion is completely decoupled from the motion along the other two directions, and by making the standard (albeit unphysical) assumption [13, [14]

$$
\frac{\partial B}{\partial z}=\text { const }>0 \text {. }
$$

whence

$$
\mu \boldsymbol{\sigma} \cdot \mathbf{B}=(b+a z) \sigma_{z}
$$

where $a>0$ and $b$ are constants. Then

$$
\Psi_{t}^{(+)}=\left(\begin{array}{c}
\Phi_{t}^{(+)}(z) \phi_{t}(x, y) \\
0
\end{array}\right) \text { and } \Psi_{t}^{(-)}=\left(\begin{array}{c}
0 \\
\Phi_{t}^{(-)}(z) \phi_{t}(x, y)
\end{array}\right)
$$

where $\Phi_{t}^{( \pm)}$are the solutions of

$$
i \hbar \frac{\partial \Phi_{t}^{( \pm)}}{\partial t}=-\frac{\hbar^{2}}{2 m} \frac{\partial^{2} \Phi_{t}^{( \pm)}}{\partial z^{2}} \mp(b+a z) \Phi_{t}^{( \pm)}
$$

for initial conditions $\Phi_{0}{ }^{( \pm)}=\Phi_{0}(z)$. Since $z$ generates translations of the $z$-component of the momentum, the behavior described above follows easily. More explicitly, the limiting behavior for $t \rightarrow \infty$ readily follows by a stationary phase argument on the explicit solution ${ }^{9}$ of (2.15).

\footnotetext{
${ }^{9}$ Eq. (2.15) is readily solved:$$
\Phi_{t}^{( \pm)}(z)=\int G^{( \pm)}\left(z, z^{\prime} ; t\right) \Phi_{0}\left(z^{\prime}\right) d z^{\prime},
$$

where (by the standard rules for the Green's function of linear and quadratic Hamiltonians)$$
G^{( \pm)}\left(z, z^{\prime} ; t\right)=\sqrt{\frac{m}{2 \pi i \hbar t}} e^{\frac{i}{\hbar}\left(\frac{m}{2 t}\left(z-z^{\prime}-( \pm) \frac{a t^{2}}{m}\right)^{2}+\frac{( \pm) a t}{2}\left(z-z^{\prime}-( \pm) \frac{a t^{2}}{m}\right)-( \pm)\left(a z^{\prime}+b\right) t+\frac{a t^{3}}{3 m}\right)}
$$ 
More simply, we may consider the initial Gaussian state

$$
\Phi_{0}=\frac{e^{\left(-\frac{z^{2}}{4 d^{2}}\right)}}{\left(2 d^{2} \pi\right)^{\frac{1}{4}}}
$$

for which $\left|\Phi_{t}^{ \pm}(z)\right|^{2}$, the probability density of the particle being at a point of $z$-coordinate $z$, is, by the linearity of the interaction in (2.15), a Gaussian with mean and mean square deviation given respectively by

$$
\bar{z}(t)=( \pm) \frac{a t^{2}}{2 m} \quad d(t)=d \sqrt{1+\frac{\hbar^{2} t^{2}}{2 m^{2} d^{4}}} .
$$

For a more general initial wave function,

$$
\Psi=\psi \otimes \Phi_{0} \quad \psi=\alpha \psi^{(+)}+\beta \psi^{(-)}
$$

passage through the magnetic field will, by linearity, split the wave function into an upwarddeflected piece (proportional to $\psi^{(+)}$) and a downward-deflected piece (proportional to $\psi^{(-)}$), with corresponding deflections of the trajectories. The outcome is registered by detectors placed in the paths of these two possible "beams." Thus of the four kinematically possible outcomes ("pointer orientations") the occurrence of no detection and of simultaneous detection can be ignored as highly unlikely, and the two relevant outcomes correspond to registration by either the upper or the lower detector. Accordingly, for a measurement of $\sigma_{z}$ the experiment is equipped with a "calibration" (i.e., an assignment of numerical values to the outcomes of the experiment) $\lambda_{+}=1$ for upper detection and $\lambda_{-}=-1$ for lower detection (while for a measurement of the $z$-component of the spin angular momentum itself the calibration is given by $\frac{1}{2} \hbar \lambda_{ \pm}$).

Note that one can completely understand what's going on in this Stern-Gerlach experiment without invoking any putative property of the electron such as its actual z-component of spin that is supposed to be revealed in the experiment. For a general initial wave function there is no such property. What is more, the transparency of the analysis of this experiment makes it clear that there is nothing the least bit remarkable (or for that matter "nonclassical") about the nonexistence of this property. But the failure to pay attention to the role of operators as observables, i.e., to precisely what we should mean when we speak of measuring operatorobservables, helps create a false impression of quantum peculiarity.

\subsection{A Remark on the Reality of Spin in Bohmian Mechanics}

Bell has said that (for Bohmian mechanics) spin is not real. Perhaps he should better have said: "Even spin is not real," not merely because of all observables, it is spin which is generally regarded as quantum mechanically most paradigmatic, but also because spin is treated in orthodox quantum theory very much like position, as a "degree of freedom" - a discrete index which supplements the continuous degrees of freedom corresponding to position - in the wave function.

Be that as it may, his basic meaning is, we believe, this: Unlike position, spin is not primitive, i.e., no actual discrete degrees of freedom, analogous to the actual positions of the particles, are added to the state description in order to deal with "particles with spin." Roughly speaking, spin is merely in the wave function. At the same time, as explained in Section 2.5. "spin measurements" are completely clear, and merely reflect the way spinor wave functions are incorporated into a description of the motion of configurations. 
In this regard, it might be objected that while spin may not be primitive, so that the result of our "spin measurement" will not reflect any initial primitive property of the system, nonetheless this result is determined by the initial configuration of the system, i.e., by the position of our electron, together with its initial wave function, and as such - as a function $X_{\sigma_{z}}(\mathbf{q}, \psi)$ of the state of the system - it is some property of the system and in particular it is surely real. We shall address this issue in Sections 8.3 and 8.4

\subsection{The Framework of Discrete Experiments}

We shall now consider a generic experiment. Whatever its significance, the information conveyed by the experiment is registered in the apparatus as an output, represented, say, by the orientation of a pointer. Moreover, when we speak of a generic experiment, we have in mind a fairly definite initial state of the apparatus, the ready state $\Phi_{0}=\Phi_{0}(y)$, one for which the apparatus should function as intended, and in particular one in which the pointer has some "null" orientation, as well as a definite initial state of the system $\psi=\psi(x)$ on which the experiment is performed. Under these conditions it turns out [25] that the initial $t=0$ wave function $\Psi_{0}=\Psi_{0}(q)$ of the composite system formed by system and apparatus, with generic configuration $q=(x, y)$, has a product form, i.e.,

$$
\Psi_{0}=\psi \otimes \Phi_{0} .
$$

Such a product form is an expression of the independence of system and apparatus immediately before the experiment begins. ${ }^{10}$

For Bohmian mechanics we should expect in general, as a consequence of the quantum equilibrium hypothesis, that the outcome of the experiment - the final pointer orientation - will be random: Even if the system and apparatus initially have definite, known wave functions, so that the outcome is determined by the initial configuration of system and apparatus, this configuration is random, since the composite system is in quantum equilibrium, and the distribution of the final configuration is given by $\left|\Psi_{T}(x, y)\right|^{2}$, where $\Psi_{T}$ is the wave function of the systemapparatus composite at the time $t=T$ when the experiment ends, and $x$, respectively $y$, is the generic system, respectively apparatus, configuration.

Suppose now that $\Psi_{T}$ has the form (2.8), which roughly corresponds to assuming that the experiment admits, i.e., that the apparatus is so designed that there is, only a finite (or countable) set of possible outcomes, given, say, by the different possible macroscopically distinct pointer orientations of the apparatus and corresponding to a partition of the apparatus configuration space into macroscopically disjoint regions $G_{\alpha}, \alpha=1,2, \ldots{ }^{11}$ We arrive in this way at the notion of discrete experiment, for which the time evolution arising from the interaction of the system and apparatus from $t=0$ to $t=T$ is given by the unitary map

$$
U: \mathcal{H} \otimes \Phi_{0} \rightarrow \bigoplus_{\alpha} \mathcal{H} \otimes \Phi_{\alpha}, \quad \psi \otimes \Phi_{0} \mapsto \Psi_{T}=\sum_{\alpha} \psi_{\alpha} \otimes \Phi_{\alpha}
$$

where $\mathcal{H}$ is the system Hilbert space of square-integrable wave functions with the usual inner

\footnotetext{
${ }^{10}$ It might be argued that it is somewhat unrealistic to assume a sharp preparation of $\psi$, as well as the possibility of resetting the apparatus always in the same initial state $\Phi_{0}$. We shall address this issue in Section 6

${ }^{11}$ Note that to assume there are only finitely, or countably, many outcomes is really no assumption at all, since the outcome should ultimately be converted to digital form, whatever its initial representation may be.
} 
product

$$
\langle\psi, \phi\rangle=\int \psi^{*}(x) \phi(x) d x
$$

and the $\Phi_{\alpha}$ are a fixed set of (normalized) apparatus states supported by the macroscopically distinct regions $G_{\alpha}$ of apparatus configurations.

The experiment usually comes equipped with an assignment of numerical values $\lambda_{\alpha}$ (or a vector of such values) to the various outcomes $\alpha$. This assignment is defined by a "calibration" function $F$ on the apparatus configuration space assuming on each region $G_{\alpha}$ the constant value $\lambda_{\alpha}$. If for simplicity we assume that these values are in one-to-one correspondence with the outcomes $^{12}$ then

$$
p_{\alpha}=\int_{F^{-1}\left(\lambda_{\alpha}\right)}\left|\Psi_{T}(x, y)\right|^{2} d x d y=\int_{G_{\alpha}}\left|\Psi_{T}(x, y)\right|^{2} d x d y
$$

is the probability of finding $\lambda_{\alpha}$, for initial system wave function $\psi$. Since $\Phi_{\alpha^{\prime}}(y)=0$ for $y \in G_{\alpha}$ unless $\alpha=\alpha^{\prime}$, we obtain

$$
p_{\alpha}=\int d x \int_{G_{\alpha}}\left|\sum_{\alpha^{\prime}} \psi_{\alpha^{\prime}}(x) \Phi_{\alpha^{\prime}}(y)\right|^{2} d y=\int\left|\psi_{\alpha}(x)\right|^{2} d x=\left\|\psi_{\alpha}\right\|^{2} .
$$

Note that when the result $\lambda_{\alpha}$ is obtained, the effective wave function of the system undergoes the transformation $\psi \rightarrow \psi_{\alpha}$.

A simple example of a discrete experiment is provided by the map

$$
U: \psi \otimes \Phi_{0} \mapsto \sum_{\alpha} c_{\alpha} \psi \otimes \Phi_{\alpha}
$$

where the $c_{\alpha}$ are complex numbers such that $\sum_{\alpha}\left|c_{\alpha}\right|^{2}=1$; then $p_{\alpha}=\left|c_{\alpha}\right|^{2}$. Note that the experiment defined by (2.21) resembles a coin-flip more than a measurement since the outcome $\alpha$ occurs with a probability independent of $\psi$.

\subsection{Reproducibility and its Consequences}

Though for a generic discrete experiment there is no reason to expect the sort of "measurementlike" behavior typical of familiar quantum measurements, there are, however, special experiments whose outcomes are somewhat less random than we might have thought possible. According to Schrödinger [2]:

The systematically arranged interaction of two systems (measuring object and measuring instrument) is called a measurement on the first system, if a directly-sensible variable feature of the second (pointer position) is always reproduced within certain error limits when the process is immediately repeated (on the same object, which in the mean time must not be exposed to additional influences).

To implement the notion of "measurement-like" experiment considered by Schrödinger, we first make some preliminary observations concerning the unitary map (2.18). Let $P_{\left[\Phi_{\alpha}\right]}$ be the

\footnotetext{
${ }^{12}$ We shall consider the more general case later on in Subsection 3.2.4.
} 
orthogonal projection in the Hilbert space $\bigoplus_{\alpha} \mathcal{H} \otimes \Phi_{\alpha}$ onto the subspace $\mathcal{H} \otimes \Phi_{\alpha}$ and let $\widetilde{\mathcal{H}_{\alpha}}$ be the subspaces of $\mathcal{H}$ defined by

$$
P_{\left[\Phi_{\alpha}\right]}\left[U\left(\mathcal{H} \otimes \Phi_{0}\right)\right]=\widetilde{\mathcal{H}_{\alpha}} \otimes \Phi_{\alpha}
$$

(Since the vectors in $\widetilde{\mathcal{H}}_{\alpha}$ arise from projecting $\Psi_{T}=\sum_{\alpha} \psi_{\alpha} \otimes \Phi_{\alpha}$ onto its $\alpha$-component, $\widetilde{\mathcal{H}}_{\alpha}$ is the space of the "collapsed" wave functions associated with the occurrence of the outcome $\alpha$.) Then

$$
U\left(\mathcal{H} \otimes \Phi_{0}\right) \subseteq \bigoplus_{\alpha} \widetilde{\mathcal{H}}_{\alpha} \otimes \Phi_{\alpha}
$$

Note, however, that it need not be the case that $U\left(\mathcal{H} \otimes \Phi_{0}\right)=\bigoplus_{\alpha} \widetilde{\mathcal{H}}_{\alpha} \otimes \Phi_{\alpha}$, and that the spaces $\widetilde{\mathcal{H}_{\alpha}}$ need be neither orthogonal nor distinct; e.g., for (2.21) $\widetilde{\mathcal{H}}_{\alpha}=\mathcal{H}$ and $U\left(\mathcal{H} \otimes \Phi_{0}\right)=$ $\mathcal{H} \otimes \sum_{\alpha} c_{\alpha} \Phi_{\alpha} \neq \bigoplus_{\alpha} \mathcal{H} \otimes \Phi_{\alpha}{ }^{13}$

A "measurement-like" experiment is one which is reproducible in the sense that it will yield the same outcome as originally obtained if it is immediately repeated. (This means in particular that the apparatus must be immediately reset to its ready state, or a fresh apparatus must be employed, while the system is not tampered with so that its initial state for the repeated experiment is its final state produced by the first experiment.) Thus the experiment is reproducible if

$$
U\left(\widetilde{\mathcal{H}_{\alpha}} \otimes \Phi_{0}\right) \subseteq \widetilde{\mathcal{H}_{\alpha}} \otimes \Phi_{\alpha}
$$

or, equivalently, if there are spaces $\mathcal{H}_{\alpha}{ }^{\prime} \subseteq \widetilde{\mathcal{H}_{\alpha}}$ such that

$$
U\left(\widetilde{\mathcal{H}_{\alpha}} \otimes \Phi_{0}\right)=\mathcal{H}_{\alpha}{ }^{\prime} \otimes \Phi_{\alpha}
$$

Note that it follows from the unitarity of $U$ and the orthogonality of the subspaces $\widetilde{\mathcal{H}_{\alpha}} \otimes \Phi_{\alpha}$ that the subspaces $\widetilde{\mathcal{H}}_{\alpha} \otimes \Phi_{0}$ and hence the $\widetilde{\mathcal{H}}_{\alpha}$ are also orthogonal. Therefore, by taking the orthogonal sum over $\alpha$ of both sides of (2.25), we obtain

$$
\bigoplus_{\alpha} U\left(\widetilde{\mathcal{H}_{\alpha}} \otimes \Phi_{0}\right)=U\left(\bigoplus_{\alpha} \widetilde{\mathcal{H}_{\alpha}} \otimes \Phi_{0}\right)=\bigoplus_{\alpha} \mathcal{H}_{\alpha}{ }^{\prime} \otimes \Phi_{\alpha}
$$

If we now make the simplifying assumption that the subspaces $\widetilde{\mathcal{H}_{\alpha}}$ are finite dimensional, we have from unitarity that $\widetilde{\mathcal{H}}_{\alpha}=\mathcal{H}_{\alpha}{ }^{\prime}$, and thus, by comparing (2.23) and (2.26), that equality holds in (2.23) and that

$$
\mathcal{H}=\bigoplus_{\alpha} \mathcal{H}_{\alpha}
$$

with

$$
U\left(\mathcal{H}_{\alpha} \otimes \Phi_{0}\right)=\mathcal{H}_{\alpha} \otimes \Phi_{\alpha}
$$

for

$$
\mathcal{H}_{\alpha} \equiv \widetilde{\mathcal{H}_{\alpha}}=\mathcal{H}_{\alpha}{ }^{\prime}
$$

\footnotetext{
${ }^{13}$ Note that if $\mathcal{H}$ has finite dimension $n$, and the number of outcomes $\alpha$ is $m$, $\operatorname{dim}\left[U\left(\mathcal{H} \otimes \Phi_{0}\right)\right]=n$, while $\operatorname{dim}\left[\bigoplus_{\alpha} \mathcal{H} \otimes \Phi_{\alpha}\right]=n \cdot m$.
} 
Therefore if the wave function of the system is initially in $\mathcal{H}_{\alpha}$, outcome $\alpha$ definitely occurs and the value $\lambda_{\alpha}$ is thus definitely obtained (assuming again for simplicity one-to-one correspondence between outcomes and results). It then follows that for a general initial system wave function

$$
\psi=\sum_{\alpha} P_{\mathcal{H}_{\alpha}} \psi
$$

where $P_{\mathcal{H}_{\alpha}}$ is the projection in $\mathcal{H}$ onto the subspace $\mathcal{H}_{\alpha}$, that the outcome $\alpha$, with result $\lambda_{\alpha}$, is obtained with (the usual) probability

$$
p_{\alpha}=\left\|P_{\mathcal{H}_{\alpha}} \psi\right\|^{2}=\left\langle\psi, P_{\mathcal{H}_{\alpha}} \psi\right\rangle,
$$

which follows from (2.28), (2.20), and (2.18) since $U\left(P_{\mathcal{H}_{\alpha}} \psi \otimes \Phi_{0}\right)=\psi_{\alpha} \otimes \Phi_{\alpha}$ and hence $\left\|P_{\mathcal{H}_{\alpha}} \psi\right\|=$ $\left\|\psi_{\alpha}\right\|$ by unitarity. In particular, when the $\lambda_{\alpha}$ are real-valued, the expected value obtained is

$$
\sum_{\alpha} p_{\alpha} \lambda_{\alpha}=\sum_{\alpha} \lambda_{\alpha}\left\|P_{\mathcal{H}_{\alpha}} \psi\right\|^{2}=\langle\psi, A \psi\rangle
$$

where

$$
A=\sum_{\alpha} \lambda_{\alpha} P_{\mathcal{H}_{\alpha}}
$$

is the self-adjoint operator with eigenvalues $\lambda_{\alpha}$ and spectral projections $P_{\mathcal{H}_{\alpha}}$.

\subsection{Operators as Observables}

What we wish to emphasize here is that, insofar as the statistics for the values which result from the experiment are concerned,

the relevant data for the experiment are the collection $\left\{\mathcal{H}_{\alpha}\right\}$ of special orthogonal subspaces, together with the corresponding calibration $\left\{\lambda_{\alpha}\right\}$,

and this data is compactly expressed and represented by the self-adjoint operator $A$, on the system Hilbert space $\mathcal{H}$, given by (2.31). Thus, under the assumptions we have made, with a reproducible experiment $\mathscr{E}$ we naturally associate an operator $A=A_{\mathscr{E}}$, a single mathematical object, defined on the system alone, in terms of which an efficient description (2.29) of the statistics of the possible results is achieved; we shall denote this association by

$$
\mathscr{E} \mapsto A
$$

If we wish we may speak of "operators as observables," and when an experiment $\mathscr{E}$ is associated with a self-adjoint operator $A$, as described above, we may say that the experiment $\mathscr{E}$ is a "measurement" of the observable represented by the self-adjoint operator $A$. If we do so, however, it is important that we appreciate that in so speaking we merely refer to what we have just derived: the role of operators in the description of certain experiments. ${ }^{14}$

So understood, the notion of operator-as-observable in no way implies that anything is genuinely measured in the experiment, and certainly not the operator itself! In a general experiment

\footnotetext{
${ }^{14}$ Operators as observables also naturally convey information about the system's wave function after the experiment. For example, for an ideal measurement, when the outcome is $\alpha$ the wave function of the system after the experiment is (proportional to) $P_{\mathcal{H}_{\alpha}} \psi$. We shall elaborate upon this in the next section.
} 
no system property is being measured, even if the experiment happens to be measurement-like. (Position measurements in Bohmian mechanics are of course an important exception.) What in general is going on in obtaining outcome $\alpha$ is completely straightforward and in no way suggests, or assigns any substantive meaning to, statements to the effect that, prior to the experiment, observable $A$ somehow had a value $\lambda_{\alpha}$-whether this be in some determinate sense or in the sense of Heisenberg's "potentiality" or some other ill-defined fuzzy sense - which is revealed, or crystallized, by the experiment. Even speaking of the observable $A$ as having value $\lambda_{\alpha}$ when the system's wave function is in $\mathcal{H}_{\alpha}$, i.e., when this wave function is an eigenstate of $A$ of eigenvalue $\lambda_{\alpha}$-insofar as it suggests that something peculiarly quantum is going on when the wave function is not an eigenstate whereas in fact there is nothing the least bit peculiar about the situation-perhaps does more harm than good.

It might be objected that we are claiming to arrive at the quantum formalism under somewhat unrealistic assumptions, such as, for example, reproducibility or finite dimensionality. We agree. But this objection misses the point of the exercise. The quantum formalism itself is an idealization; when applicable at all, it is only as an approximation. Beyond illuminating the role of operators as ingredients in this formalism, our point was to indicate how naturally it emerges. In this regard we must emphasize that the following question arises for quantum orthodoxy, but does not arise for Bohmian mechanics: For precisely which theory is the quantum formalism an idealization?

We shall discuss how to go beyond the idealization involved in the quantum formalism in Section 4-after having analyzed it thoroughly in Section 3. First we wish to show that many more experiments than those satisfying our assumptions can indeed be associated with operators in exactly the manner we have described.

\subsection{The General Framework of Bohmian Experiments}

According to (2.19) the statistics of the results of a discrete experiment are governed by the probability measure $\rho_{\Psi_{T}} \circ F^{-1}$, where $\rho_{\Psi_{T}}(d q)=\left|\Psi_{T}(q)\right|^{2} d q$ is the quantum equilibrium measure. Note that discreteness of the value space of $F$ plays no role in the characterization of this measure. This suggests that we may consider a more general notion of experiment, not based on the assumption of a countable set of outcomes, but only on the unitarity of the operator $U$, which transforms the initial state $\psi \otimes \Phi_{0}$ into the final state $\Psi_{T}$, and on a generic calibration function $F$ from the configuration space of the composite system to some value space, e.g., $\mathbb{R}$, or $\mathbb{R}^{m}$, giving the result of the experiment as a function $F\left(Q_{T}\right)$ of the final configuration $Q_{T}$ of system and apparatus. We arrive in this way at the notion of general experiment

$$
\mathscr{E} \equiv\left\{\Phi_{0}, U, F\right\}
$$

where the unitary $U$ embodies the interaction of system and apparatus and the function $F$ could be completely general. Of course, for application to the results of real-world experiments $F$ might represent the "orientation of the apparatus pointer" or some coarse-graining thereof.

Performing $\mathscr{E}$ on a system with initial wave function $\psi$ leads to the result $Z=F\left(Q_{T}\right)$ and since $Q_{T}$ is randomly distributed according to the quantum equilibrium measure $\rho_{\Psi_{T}}$, the probability distribution of $Z$ is given by the induced measure

$$
\rho_{\psi}^{Z}=\rho_{\Psi_{T}} \circ F^{-1}
$$


(We have made explicit only the dependence of the measure on $\psi$, since the initial apparatus state $\Phi_{0}$ is of course fixed, defined by the experiment $\mathscr{E}$.) Note that this more general notion of experiment eliminates the slight vagueness arising from the imprecise notion of macroscopic upon which the notion of discrete experiment is based. Note also that the structure (2.34) conveys information about the wave function (2.6) of the system after a certain result $F\left(Q_{T}\right)$ is obtained.

Note, however, that this somewhat formal notion of experiment may not contain enough information to determine the detailed Bohmian dynamics, which would require specification of the Hamiltonian of the system-apparatus composite, that might not be captured by $U$. In particular, the final configuration $Q_{T}$ may not be determined, for given initial wave function, as a function of the initial configuration of system and apparatus. $\mathscr{E}$ does, however, determine what is relevant for our purposes about the random variable $Q_{T}$, namely its distribution, and hence that of $Z=F\left(Q_{T}\right)$.

Let us now focus on the right had side of the equation (2.29), which establishes the association of operators with experiments: $\left\langle\psi, P_{\mathcal{H}_{\alpha}} \psi\right\rangle$ is the probability that "the operator $A$ has value $\lambda_{\alpha}$ ", and according to standard quantum mechanics the statistics of the results of measuring a general self-adjoint operator $A$, not necessarily with pure point spectrum, in the (normalized) state $\psi$ are described by the probability measure

$$
\Delta \mapsto \mu_{\psi}^{A}(\Delta) \equiv\left\langle\psi, P^{A}(\Delta) \psi\right\rangle
$$

where $\Delta$ is a (Borel) set of real numbers and $P^{A}: \Delta \mapsto P^{A}(\Delta)$ is the projection-valued-measure (PVM) uniquely associated with $A$ by the spectral theorem. (We recall [70] that a PVM is a normalized, countably additive set function whose values are, instead of nonnegative reals, orthogonal projections on a Hilbert space $\mathcal{H}$. Any PVM $P$ on $\mathcal{H}$ determines, for any given $\psi \in \mathcal{H}$, a probability measure $\mu_{\psi} \equiv \mu_{\psi}^{P}: \Delta \mapsto\langle\psi, P(\Delta) \psi\rangle$ on $\mathbb{R}$. Integration against projection-valuedmeasure is analogous to integration against ordinary measures, so that $B \equiv \int f(\lambda) P(d \lambda)$ is welldefined, as an operator on $\mathcal{H}$. Moreover, by the spectral theorem every self-adjoint operator $A$ is of the form $A=\int \lambda P(d \lambda)$, for a unique projection-valued-measure $P=P^{A}$, and $\int f(\lambda) P(d \lambda)=$ $f(A)$. )

It is then rather clear how (2.33) extends to general self-adjoint operators: a general experiment $\mathscr{E}$ is a measurement of the self-adjoint operator $A$ if the statistics of the results of $\mathscr{E}$ are given by (2.36), i.e.,

$$
\mathscr{E} \mapsto A \quad \text { if and only if } \quad \rho_{\psi}^{Z}=\mu_{\psi}^{A} .
$$

In particular, if $\mathscr{E} \mapsto A$, then the moments of the result of $\mathscr{E}$ are the moments of $A$ :

$$
<Z^{n}>=\int \lambda^{n}\langle\psi, P(d \lambda) \psi\rangle=\left\langle\psi, A^{n} \psi\right\rangle
$$

\section{The Quantum Formalism}

The spirit of this section will be rather different from that of the previous one. Here the focus will be on the formal structure of experiments measuring self-adjoint operators. Our aim is to show that the standard quantum formalism emerges from a formal analysis of the association $\mathscr{E} \mapsto A$ between operator and experiment provided by (2.37). By "formal analysis" we mean not only that the detailed physical conditions under which might $\mathscr{E} \mapsto A$ hold (e.g., reproducibility) 
will play no role, but also that the practical requirement that $\mathscr{E}$ be physically realizable will be of no relevance whatsoever.

Note that such a formal approach is unavoidable in order to recover the quantum formalism. In fact, within the quantum formalism one may consider measurements of arbitrary self-adjoint operators, for example, the operator $A=\hat{X}^{2} \hat{P}+\hat{P} X^{2}$, where $\hat{X}$ and $\hat{P}$ are respectively the position and the momentum operators. However, it may very well be the case that no "real world" experiment measuring $A$ exists. Thus, in order to allow for measurements of arbitrary selfadjoint operators we shall regard (2.34) as characterizing an "abstract experiment"; in particular, we shall not regard the unitary map $U$ as arising necessarily from a (realizable) Schrödinger time evolution. We may also speak of virtual experiments.

In this regard one should observe that to resort to a formal analysis is indeed quite common in physics. Consider, e.g., the Hamiltonian formulation of classical mechanics that arose from an abstraction of the physical description of the world provided by Newtonian mechanics. Here we may freely speak of completely general Hamiltonians, e.g. $H(p, q)=p^{6}$, without being concerned about whether they are physical or not. Indeed, only very few Hamiltonians correspond to physically realizable motions!

A warning: As we have stressed in the introduction and in Section 2.9, when we speak here of a measurement we don't usually mean a genuine measurement - an experiment revealing the pre-existing value of a quantity of interest, the measured quantity or property. (We speak in this unfortunate way because it is standard.) Genuine measurement will be discussed much later, in Section 7 .

\subsection{Weak Formal Measurements}

The first formal notion we shall consider is that of weak formal measurement, formalizing the relevant data of an experiment measuring a self-adjoint operator:

Any orthogonal decomposition $\mathcal{H}=\bigoplus_{\alpha} \mathcal{H}_{\alpha}$, i.e., any complete collection $\left\{\mathcal{H}_{\alpha}\right\}$ of mutually orthogonal subspaces, paired with any set $\left\{\lambda_{\alpha}\right\}$ of distinct real numbers, defines the weak formal measurement $\mathcal{M} \equiv\left\{\left(\mathcal{H}_{\alpha}, \lambda_{\alpha}\right)\right\} \equiv\left\{\mathcal{H}_{\alpha}, \lambda_{\alpha}\right\}$.

(Compare (3.1) with (2.32) and note that now we are not assuming that the spaces $\mathcal{H}_{\alpha}$ are finite-dimensional.) The notion of weak formal measurement is aimed at expressing the minimal structure that all experiments (some or all of which might be virtual) measuring the same operator $A=\sum \lambda_{\alpha} P_{\mathcal{H}_{\alpha}}$ have in common $\left(P_{\mathcal{H}_{\alpha}}\right.$ is the orthogonal projection onto the subspace $\left.\mathcal{H}_{\alpha}\right)$. Then, "to perform $\mathcal{M}$ " shall mean to perform (at least virtually) any one of these experiments, i.e., any experiment such that

$$
p_{\alpha}=\left\langle\psi, P_{\mathcal{H}_{\alpha}} \psi\right\rangle
$$

is the probability of obtaining the result $\lambda_{\alpha}$ on a system initially in the state $\psi$. (This is of course equivalent to requiring that the result $\lambda_{\alpha}$ is definitely obtained if and only if the initial wave function $\psi \in \mathcal{H}_{\alpha}$.)

Given $\mathcal{M} \equiv\left\{\mathcal{H}_{\alpha}, \lambda_{\alpha}\right\}$ consider the set function

$$
P: \Delta \mapsto P(\Delta) \equiv \sum_{\lambda_{\alpha} \in \Delta} P_{\mathcal{H}_{\alpha}}
$$

where $\Delta$ is a set of real numbers (technically, a Borel set). Then 
1) $P$ is normalized, i.e., $P(\mathbb{R})=I$, where $I$ is the identity operator and $\mathbb{R}$ is the real line,

2) $P(\Delta)$ is an orthogonal projection, i.e., $P(\Delta)^{2}=P(\Delta)=P(\Delta)^{*}$,

3) $P$ is countably additive, i.e., $P\left(\bigcup_{n} \Delta_{n}\right)=\sum_{n} P\left(\Delta_{n}\right)$, for $\Delta_{n}$ disjoint sets.

Thus $P$ is a projection-valued-measure and therefore the notion of weak formal measurement is indeed equivalent to that of "discrete" PVM, that is, a PVM supported by a countable set $\left\{\lambda_{\alpha}\right\}$ of values.

More general PVMs, e.g. PVMs supported by a continuous set of values, will arise if we extend (3.1) and base the notion of weak formal measurement upon the general association (2.37) between experiments and operators. If we stipulate that

any projection-valued-measure $P$ on $\mathcal{H}$ defines a weak formal measurement $\mathcal{M} \equiv P$,

then "to perform $\mathcal{M}$ " shall mean to perform any experiment $\mathscr{E}$ associated with $A=\int \lambda P(d \lambda)$ in the sense of (2.37).

Note that since by the spectral theorem there is a natural one-to-one correspondence between PVMs and self-adjoint operators, we may speak equivalently of the operator $A=A_{\mathcal{M}}$, for given $\mathcal{M}$, or of the weak formal $\mathcal{M}=\mathcal{M}_{A}$, for given $A$. In particular, the weak formal measurement $\mathcal{M}_{A}$ represents the equivalence class of all experiments $\mathscr{E} \rightarrow A$.

\subsection{Strong Formal Measurements}

We wish now to classify the different experiments $\mathscr{E}$ associated with the same self-adjoint operator $A$ by taking into account the effect of $\mathscr{E}$ on the state of the system, i.e., the state transformations $\psi \rightarrow \psi_{\alpha}$ induced by the occurrence of the various results $\lambda_{\alpha}$ of $\mathscr{E}$. Accordingly, unless otherwise stated, from now on we shall assume $\mathscr{E}$ to be a discrete experiment measuring $A=\sum \lambda_{\alpha} P_{\mathcal{H}_{\alpha}}$, for which the state transformation $\psi \rightarrow \psi_{\alpha}$ is defined by (2.18). This leads to the notion of strong formal measurements. For the most important types of strong formal measurements, ideal, normal and standard, there is a one-to-one correspondence between $\alpha$ 's and numerical results $\lambda_{\alpha}$.

\subsubsection{Ideal Measurements}

Given a weak formal measurement of $A$, the simplest possibility for the transition $\psi \rightarrow \psi_{\alpha}$ is that when the result $\lambda_{\alpha}$ is obtained, the initial state $\psi$ is projected onto the corresponding space $\mathcal{H}_{\alpha}$, i.e., that

$$
\psi \rightarrow \psi_{\alpha}=P_{\mathcal{H}_{\alpha}} \psi \text {. }
$$

This prescription defines uniquely the ideal measurement of $A$. (The transformation $\psi \rightarrow \psi_{\alpha}$ should be regarded as defined only in the projective sense: $\psi \rightarrow \psi_{\alpha}$ and $\psi \rightarrow c \psi_{\alpha}(c \neq 0)$ should be regarded as the same transition.) "To perform an ideal measurement of $A$ " shall then mean to perform a discrete experiment $\mathscr{E}$ whose results are statistically distributed according to (3.2) and whose state transformations (2.18) are given by (3.5).

Under an ideal measurement the wave function changes as little as possible: an initial $\psi \in \mathcal{H}_{\alpha}$ is unchanged by the measurement. Ideal measurements have always played a privileged role in quantum mechanics. It is the ideal measurements that are most frequently discussed in textbooks. 
It is for ideal measurements that the standard collapse rule is obeyed. When Dirac [23] wrote: "a measurement always causes the system to jump into an eigenstate of the dynamical variable that is being measured" he was referring to an ideal measurement.

\subsubsection{Normal Measurements}

The rigid structure of ideal measurements can be weakened by requiring only that $\mathcal{H}_{\alpha}$ as a whole, and not the individual vectors in $\mathcal{H}_{\alpha}$, is unchanged by the measurement and therefore that the state transformations induced by the measurement are such that when the result $\lambda_{\alpha}$ is obtained the transition

$$
\psi \rightarrow \psi_{\alpha}=U_{\alpha} P_{\mathcal{H}_{\alpha}} \psi
$$

occurs, where the $U_{\alpha}$ are operators on $\mathcal{H}_{\alpha}\left(U_{\alpha}: \mathcal{H}_{\alpha} \rightarrow \mathcal{H}_{\alpha}\right)$. Then for any such discrete experiment $\mathscr{E}$ measuring $A$, the $U_{\alpha}$ can be chosen so that (3.6) agrees with (2.18), i.e., so that for $\psi \in \mathcal{H}_{\alpha}, U\left(\psi \otimes \Phi_{0}\right)=U_{\alpha} \psi \otimes \Phi_{\alpha}$, and hence so that $U_{\alpha}$ is unitary (or at least a partial isometry). Such a measurement, with unitaries $U_{\alpha}: \mathcal{H}_{\alpha} \rightarrow \mathcal{H}_{\alpha}$, will be called a normal measurement of $A$.

In contrast with an ideal measurement, a normal measurement of an operator is not uniquely determined by the operator itself: additional information is needed to determine the transitions, and this is provided by the family $\left\{U_{\alpha}\right\}$. Different families define different normal measurements of the same operator. Note that ideal measurements are, of course, normal (with $U_{\alpha}=I_{\alpha} \equiv$ identity on $\mathcal{H}_{\alpha}$ ), and that normal measurements with one-dimensional subspaces $\mathcal{H}_{\alpha}$ are necessarily ideal.

Since the transformations (3.6) leave invariant the subspaces $\mathcal{H}_{\alpha}$, the notion of normal measurement characterizes completely the class of reproducible measurements of self-adjoint operators. Following the terminology introduced by Pauli [66], normal measurement are sometimes called measurements of first kind. Normal measurements are also quantum non demolition (QND) measurements [18, defined as measurements such that the operators describing the induced state transformations, i.e, the operators $R_{\alpha} \equiv U_{\alpha} P_{\mathcal{H}_{\alpha}}$, commute with the measured operator $A=\sum \lambda_{\alpha} P_{\mathcal{H}_{\alpha}}$. (This condition is regarded as expressing that the measurement leaves the measured observable $A$ unperturbed).

\subsubsection{Standard Measurements}

We may now drop the condition that the $\mathcal{H}_{\alpha}$ are left invariant by the measurement and consider the very general state transformations

$$
\psi \rightarrow \psi_{\alpha}=T_{\alpha} P_{\mathcal{H}_{\alpha}} \psi
$$

with operators $T_{\alpha}: \mathcal{H}_{\alpha} \rightarrow \mathcal{H}$. Then, exactly as for the case of normal measurements, it follows that $T_{\alpha}$ can be chosen to be unitary from $\mathcal{H}_{\alpha}$ onto its range $\widetilde{\mathcal{H}}_{\alpha}$. The subspaces $\widetilde{\mathcal{H}}_{\alpha}$ need be neither orthogonal nor distinct. We shall write $R_{\alpha}=T_{\alpha} P_{\mathcal{H}_{\alpha}}$ for the general transition operators. With $T_{\alpha}$ as chosen, $R_{\alpha}$ is characterized by the equation $R_{\alpha}^{*} R_{\alpha}=P_{\mathcal{H}_{\alpha}}$ (where $R_{\alpha}^{*}$ denotes the adjoint of $R_{\alpha}$ ).

The state transformations (3.7), given by unitaries $T_{\alpha}: \mathcal{H}_{\alpha} \rightarrow \widetilde{\mathcal{H}_{\alpha}}$, or equivalently by bounded operators $R_{\alpha}$ on $\mathcal{H}$ satisfying $R_{\alpha}^{*} R_{\alpha}=P_{\mathcal{H}_{\alpha}}$, define what we shall call a standard measurement of

A. Note that normal measurements are standard measurements with $\widetilde{\mathcal{H}_{\alpha}}=\mathcal{H}_{\alpha}$ (or $\widetilde{\mathcal{H}_{\alpha}} \subset \mathcal{H}_{\alpha}$ ). Although standard measurements are in a sense more realistic than normal measurements (real 
world measurements are seldom reproducible in a strict sense), they are very rarely discussed in textbooks. We emphasize that the crucial data in a standard measurement is given by $R_{\alpha}$, which governs both the state transformations $\left(\psi \rightarrow R_{a} \psi\right)$ and the probabilities $\left(p_{\alpha}=\left\langle\psi, P_{\mathcal{H}_{\alpha}} \psi\right\rangle=\right.$ $\left.\left\|R_{\alpha} \psi\right\|^{2}\right)$.

We shall illustrate the main features of standard measurements by considering a very simple example: Let $\left\{e_{0}, e_{1}, e_{2}, \ldots\right\}$, be a fixed orthonormal basis of $\mathcal{H}$ and consider the standard measurement whose results are the numbers $0,1,2, \ldots$ and whose state transformations are defined by the operators

$$
R_{\alpha} \equiv\left|e_{0}\right\rangle\left\langle e_{\alpha}\right| \quad \text { i.e., } \quad R_{\alpha} \psi=\left\langle e_{\alpha}, \psi\right\rangle e_{0}, \quad \alpha=0,1,2, \ldots
$$

With such $R_{\alpha}$ 's are associated the projections $P_{\alpha}=R_{\alpha}^{*} R_{\alpha}=\left|e_{\alpha}\right\rangle\left\langle e_{\alpha}\right|$, i.e., the projections onto the one dimensional spaces $\mathcal{H}_{\alpha}$ spanned respectively by the vectors $e_{\alpha}$. Thus, this is a measurement of the operator $A=\sum_{\alpha} \alpha\left|e_{\alpha}\right\rangle\left\langle e_{\alpha}\right|$. Note that the spaces $\widetilde{\mathcal{H}_{\alpha}}$, i.e. the ranges of the $R_{\alpha}$ 's, are all the same and equal to the space $\mathcal{H}_{0}$ generated by the vector $e_{0}$. The measurement is then not normal since $\mathcal{H}_{\alpha} \neq \widetilde{\mathcal{H}}_{\alpha}$. Finally, note that this measurement could be regarded as giving a simple model for a photo detection experiment, where any state is projected onto the "vacuum state" $e_{0}$ after the detection.

\subsubsection{Strong Formal Measurements}

We shall now relax the condition that $\alpha \mapsto \lambda_{\alpha}$ is one-to-one, as we would have to do for an experiment having a general calibration $\alpha \mapsto \lambda_{\alpha}$, which need not be invertible. This leads to (what we shall call) a strong formal measurement. Since this notion provides the most general formalization of the notion of a "measurement of a self-adjoint operator" that takes into account the effect of the measurement on the state of the system, we shall spell it out precisely as follows:

Any complete (labelled) collection $\left\{\mathcal{H}_{\alpha}\right\}$ of mutually orthogonal subspaces, any (labelled) set $\left\{\lambda_{\alpha}\right\}$ of not necessarily distinct real numbers, and any (labelled) collec-

tion $\left\{R_{\alpha}\right\}$ of bounded operators on $\mathcal{H}$, such that $R_{\alpha}^{*} R_{\alpha} \equiv P_{\mathcal{H}_{\alpha}}$ (the projection onto

$\left.\mathcal{H}_{\alpha}\right)$, defines a strong formal measurement.

A strong formal measurement will be compactly denoted by $\mathcal{M} \equiv\left\{\left(\mathcal{H}_{\alpha}, \lambda_{\alpha}, R_{\alpha}\right)\right\} \equiv$ $\left\{\mathcal{H}_{\alpha}, \lambda_{\alpha}, R_{\alpha}\right\}$, or even more compactly by $\mathcal{M} \equiv\left\{\lambda_{\alpha}, R_{\alpha}\right\}$ (the spaces $\mathcal{H}_{\alpha}$ can be extracted from the projections $P_{\mathcal{H}_{\alpha}}=R_{\alpha}^{*} R_{\alpha}$ ). With $\mathcal{M}$ is associated the operator $A=\sum \lambda_{\alpha} P_{\mathcal{H}_{\alpha}}$. Note that since the $\lambda_{\alpha}$ are not necessarily distinct numbers, $P_{\mathcal{H}_{\alpha}}$ need not be the spectral projection $P^{A}\left(\lambda_{\alpha}\right)$ associated with $\lambda_{\alpha}$; in general

$$
P^{A}(\lambda)=\sum_{\alpha: \lambda_{\alpha}=\lambda} P_{\mathcal{H}_{\alpha}}
$$

i.e., it is the sum of all the $P_{\mathcal{H}_{\alpha}}$ 's that are associated with the value $\lambda .{ }^{15}$ "To perform the measurement $\mathcal{M}$ " on a system initially in $\psi$ shall accordingly mean to perform a discrete experiment $\mathscr{E}$ such that: 1) the probability $p(\lambda)$ of getting the result $\lambda$ is governed by $A$, i.e.,

\footnotetext{
${ }^{15}$ It is for this reason that it would be pointless and inappropriate to similarly generalize weak measurements. It is only when the state transformation is taken into account that the distinction between the outcome $\alpha$ (which determines the transformation) and the result $\lambda_{\alpha}$ (whose probability the formal measurement is to supply) becomes relevant.
} 
$p(\lambda)=\left\langle\psi, P^{A}(\lambda) \psi\right\rangle$, and 2) the state transformations of $\mathscr{E}$ are those prescribed by $\mathcal{M}$, i.e., $\psi \rightarrow \psi_{\alpha}=R_{\alpha} \psi$.

Observe that strong formal measurements do provide a more realistic formalization of the notion of measurement of an operator than standard measurements: the notion of discrete experiment does not imply a one-to-one correspondence between outcomes, i.e, final macroscopic configurations of the pointer, and the numerical results of the experiment.

The relationship between (weak or strong) formal measurements, self-adjoint operators, and experiments can be summarized by the following sequence of maps:

$$
\mathscr{E} \mapsto \mathcal{M} \mapsto A
$$

The first map expresses that $\mathcal{M}$ (weak or strong) is a formalization of $\mathscr{E}$ - it contains the "relevant data" about $\mathscr{E}$ - and it will be many-to-one if $\mathcal{M}$ is a weak formal measurement ${ }^{16}$; the second map expresses that $\mathcal{M}$ is a formal measurement of $A$ and it will be many-to-one if $\mathcal{M}$ is (required to be) strong and one-to-one if $\mathcal{M}$ is weak. Note that $\mathscr{E} \mapsto A$ is always many-to-one.

\subsection{From Formal Measurements to Experiments}

Given a strong measurement $\mathcal{M} \equiv\left\{\mathcal{H}_{\alpha}, \lambda_{\alpha}, R_{\alpha}\right\}$ one may easily construct a map (2.18) defining a discrete experiment $\mathscr{E}=\mathscr{E}_{\mathcal{M}}$ associated with $\mathcal{M}$ :

$$
U: \psi \otimes \Phi_{0} \mapsto \sum_{\alpha}\left(R_{\alpha} \psi\right) \otimes \Phi_{\alpha}
$$

The unitarity of $U$ ( from $\mathcal{H} \otimes \Phi_{0}$ onto the range of $U$ ) follows then immediately from the orthonormality of the $\left\{\Phi_{\alpha}\right\}$ since

$$
\sum_{\alpha}\left\|R_{\alpha} \psi\right\|^{2}=\sum_{\alpha}\left\langle\psi, R_{\alpha}^{*} R_{\alpha} \psi\right\rangle=\left\langle\psi, \sum_{\alpha} P_{\mathcal{H}_{\alpha}} \psi\right\rangle=\langle\psi, \psi\rangle=\|\psi\|^{2}
$$

This experiment is abstractly characterized by: 1) the finite or countable set $I$ of outcomes $\alpha$, 2) the apparatus ready state $\Phi_{0}$ and the set $\left\{\Phi_{\alpha}\right\}$ of normalized apparatus states, 3) the unitary map $U: \mathcal{H} \otimes \Phi_{0} \rightarrow \bigoplus_{\alpha} \mathcal{H} \otimes \Phi_{\alpha}$ given by (3.10), 4) the calibration $\alpha \mapsto \lambda_{\alpha}$ assigning numerical values (or a vector of such values) to the various outcomes $\alpha$. Note that $U$ need not arise from a Schrödinger Hamiltonian governing the interaction between system and apparatus. Thus $\mathscr{E}$ should properly be regarded as an "abstract" experiment as we have already pointed out in the introduction to this section.

\subsection{Von Neumann Measurements}

We shall now briefly comment on the relation between our approach, based on formal measurements, and the widely used formulation of quantum measurement in terms of von Neumann measurements [74].

A von Neumann measurement of $A=\sum \lambda_{\alpha} P_{\mathcal{H}_{\alpha}}$ on a system initially in the state $\psi$ can be described as follows (while the nondegeneracy of the eigenvalues of $A$-i.e., that $\operatorname{dim}\left(\mathcal{H}_{\alpha}\right)=1-$ is usually assumed, we shall not do so): Assume that the (relevant) configuration space of the

\footnotetext{
${ }^{16}$ There is an obvious natural unitary equivalence between the preimages $\mathscr{E}$ of a strong formal measurement $\mathcal{M}$.
} 
apparatus, whose generic configuration shall be denoted by $y$, is one-dimensional, so that its Hilbert space $\mathcal{H}_{\mathcal{A}} \simeq L^{2}(\mathbb{R})$, and that the interaction between system and apparatus is governed by the Hamiltonian

$$
H=H_{\mathrm{vN}}=\gamma A \otimes \hat{P}_{y}
$$

where $\hat{P}_{y} \equiv i \hbar \partial / \partial y$ is (minus) the momentum operator of the apparatus. Let $\Phi_{0}=\Phi_{0}(y)$ be the ready state of the apparatus. Then for $\psi=P_{\mathcal{H}_{\alpha}} \psi$ one easily sees that the unitary operator $U \equiv e^{-i T H / \hbar}$ transforms the initial state $\psi_{\alpha} \otimes \Phi_{0}$ into $\psi_{\alpha} \otimes \Phi_{\alpha}$ where $\Phi_{\alpha}=\Phi_{0}\left(y-\lambda_{\alpha} \gamma T\right)$, so that the action of $U$ on general $\psi=\sum P_{\mathcal{H}_{\alpha}} \psi$ is

$$
U: \psi \otimes \Phi_{0} \rightarrow \sum_{\alpha}\left(P_{\mathcal{H}_{\alpha}} \psi\right) \otimes \Phi_{\alpha}
$$

If $\Phi_{0}$ has sufficiently narrow support, say around $y=0$, the $\Phi_{\alpha}$ will have disjoint support around the "pointer positions" $y_{\alpha}=\lambda_{\alpha} \gamma T$, and thus will be orthogonal, so that, with calibration $F(y)=y / \gamma T$ (more precisely, $F(y)=y_{\alpha} / \gamma T$ for $y$ in the support of $\Phi_{\alpha}$ ), the resulting von Neumann measurement becomes a discrete experiment measuring $A$; comparing (3.13) and (3.5) we see that it is an ideal measurement of $A .^{17}$

Thus, the framework of von Neumann measurements is less general than that of discrete experiments, or equivalently of strong formal measurements; at the same time, since the Hamiltonian $H_{\mathrm{vN}}$ is not of Schrödinger type, von Neumann measurements are just as formal. (We note that more general von Neumann measurements of $A$ can be obtained by replacing $H_{\mathrm{vN}}$ with more general Hamiltonians; for example, $H_{\mathrm{vN}}^{\prime}=H_{0}+H_{\mathrm{vN}}$, where $H_{0}$ is a self-adjoint operator on the system Hilbert space which commutes with $A$, gives rise to a normal measurement of $A$, with $R_{\alpha}=e^{-i T H_{0} / \hbar} P_{\mathcal{H}_{\alpha}}$. Thus by proper extension of the von Neumann measurements one may arrive at a framework of measurements completely equivalent to that of strong formal measurements.)

\subsection{Preparation Procedures}

Before discussing further extensions of the association between experiments and operators, we shall comment on an implicit assumption apparently required for the measurement analysis to be relevant: that the system upon which measurements are to be performed can be prepared in any prescribed state $\psi$.

Firstly, we observe that the system can be prepared in a prescribed state $\psi$ by means of an appropriate standard measurement $\mathcal{M}$ performed on the system when it is initially in an unknown state $\psi^{\prime}$. We have to choose $\mathcal{M} \equiv\left\{\mathcal{H}_{\alpha}, \lambda_{\alpha}, R_{\alpha}\right\}$ in such a way that $R_{\alpha_{0}} \psi^{\prime}=\psi$, for some $\alpha_{0}$ and all $\psi^{\prime}$, i.e., that $\operatorname{Ran}\left(R_{\alpha_{0}}\right)=\operatorname{span}(\psi)$; then from reading the result $\lambda_{\alpha_{0}}$ we may infer that the system has collapsed to the state $\psi$. The simplest possibility is for $\mathcal{M}$ to be an ideal measurement with at least a one-dimensional subspace $\mathcal{H}_{\alpha_{0}}$ that is spanned by $\psi$. Another possibility is to perform a (nonideal) standard measurement like that of the example at the end of Section 3.2.3, which can be regarded as defining a preparation procedure for the state $e_{0}$.

Secondly, we wish to emphasize that the existence of preparation procedures is not as crucial for relevance as it may seem. If we had only statistical knowledge about the initial state $\psi$, nothing would change in our analysis of Bohmian experiments of Section 2, and in our conclusions

\footnotetext{
${ }^{17}$ It is usually required that von Neumann measurements be impulsive ( $\gamma$ large, $T$ small) so that only the interaction term (3.12) contributes significantly to the total Hamiltonian over the course of the measurement.
} 
concerning the emergence of self-adjoint operators, except that the uncertainty about the final configuration of the pointer would originate from both quantum equilibrium and randomness in $\psi$. We shall elaborate upon this later when we discuss Bohmian experiments for initial states described by a density matrix.

\subsection{Measurements of Commuting Families of Operators}

As hinted in Section 2.7, the result of an experiment $\mathscr{E}$ might be more complex than we have suggested until now in Section 3: it might be given by the vector $\lambda_{\alpha} \equiv\left(\lambda_{\alpha}^{(1)}, \ldots, \lambda_{\alpha}^{(m)}\right)$ corresponding to the orientations of $m$ pointers. For example, the apparatus itself may be a composite of $m$ devices with the possible results $\lambda_{\alpha}^{(i)}$ corresponding to the final state of the $i$-th device. Nothing much will change in our discussion of measurements if we now replace the numbers $\lambda_{\alpha}$ with the vectors $\lambda_{\alpha} \equiv\left(\lambda_{\alpha}^{(1)}, \ldots, \lambda_{\alpha}^{(m)}\right)$, since the dimension of the value space was not very relevant. However $\mathscr{E}$ will now be associated, not with a single self-adjoint operator, but with a commuting family of such operators. In other words, we arrive at the notion of an experiment $\mathscr{E}$ that is a measurement of a commuting family of self-adjoint operators, ${ }^{18}$ namely the family

$$
A \equiv \sum_{\alpha} \lambda_{\alpha} P_{\mathcal{H}_{\alpha}}=\left(\sum_{\alpha} \lambda_{\alpha}^{(1)} P_{\mathcal{H}_{\alpha}}, \ldots, \sum_{\alpha} \lambda_{\alpha}^{(m)} P_{\mathcal{H}_{\alpha}}\right) \equiv\left(A_{1}, \ldots, A_{m}\right) .
$$

Then the notions of the various kinds of formal measurements - weak, ideal, normal, standard, strong - extend straightforwardly to formal measurements of commuting families of operators. In particular, for the general notion of weak formal measurement given by 3.4, $P$ becomes a PVM on $\mathbb{R}^{m}$, with associated operators $A_{i}=\int_{\mathbb{R}^{m}} \lambda^{(i)} P(d \lambda) \quad\left[\lambda=\left(\lambda^{(1)}, \ldots, \lambda^{(m)}\right) \in \mathbb{R}^{m}\right]$. And just as for PVMs on $\mathbb{R}$ and self-adjoint operators, this association in fact yields, by the spectral theorem, a one-to-one correspondence between PVMs on $\mathbb{R}^{m}$ and commuting families of $m$ self-adjoint operators. The PVM corresponding to the commuting family $\left(A_{1}, \ldots, A_{m}\right)$ is in fact simply the product PVM $P=P^{A}=P^{A_{1}} \times \cdots \times P^{A_{m}}$ given on product sets by

$$
P^{A}\left(\Delta_{1} \times \cdots \times \Delta_{m}\right)=P^{A_{1}}\left(\Delta_{1}\right) \cdots P^{A_{m}}\left(\Delta_{m}\right),
$$

where $P^{A_{1}}, \ldots, P^{A_{m}}$ are the PVMs of $A_{1}, \ldots, A_{m}$, and $\Delta_{i} \subset \mathbb{R}$, with the associated probability distributions on $\mathbb{R}^{m}$ given by the spectral measures for $A$

$$
\mu_{\psi}^{A}(\Delta)=\left\langle\psi, P^{A}(\Delta) \psi\right\rangle
$$

for any (Borel) set $\Delta \subset \mathbb{R}^{m}$.

\footnotetext{
${ }^{18}$ We recall some basic facts about commuting families of self-adjoint operators [74, 71, 68]. The self-adjoint operators $A_{1}, \ldots, A_{m}$ form a commuting family if they are bounded and pairwise commute, or, more generally, if this is so for their spectral projections, i.e., if $\left[P^{A_{i}}(\Delta), P^{A_{j}}(\Gamma)\right]=0$ for all $i, j=1, \ldots, m$ and (Borel) sets $\Delta, \Gamma \subset \mathbb{R}$. A commuting family $A \equiv\left(A_{1}, \ldots, A_{m}\right)$ of self-adjoint operators is called complete if every self-adjoint operator $C$ that commutes with all members of the family can be expressed as $C=g\left(A_{1}, A_{2}, \ldots\right)$ for some function $g$. The set of all such operators cannot be extended in any suitable sense (it is closed in all relevant operator topologies). For any commuting family $\left(A_{1}, \ldots, A_{m}\right)$ of self-adjoint operators there is a self-adjoint operator $B$ and measurable functions $f_{i}$ such that $A_{i}=f_{i}(B)$. If the family is complete, then this operator has simple (i.e., nondegenerate) spectrum.
} 
In particular, for a PVM on $\mathbb{R}^{m}$, corresponding to $A=\left(A_{1}, \ldots, A_{m}\right)$, the $i$-marginal distribution, i.e., the distribution of the $i$-th component $\lambda^{(i)}$, is

$$
\mu_{\psi}^{A}\left(\mathbb{R} \times \cdots \mathbb{R} \times \Delta_{i} \times \mathbb{R} \times \cdots \times \mathbb{R}\right)=\left\langle\psi, P^{A_{i}}\left(\Delta_{i}\right) \psi\right\rangle=\mu_{\psi}^{A_{i}}\left(\Delta_{i}\right),
$$

the spectral measure for $A_{i}$. Thus, by focusing on the respective pointer variables $\lambda^{(i)}$, we may regard an experiment measuring (or a weak formal measurement of) $A=\left(A_{1}, \ldots, A_{m}\right.$ ) as providing an experiment measuring (or a weak formal measurement of) each $A_{i}$, just as would be the case for a genuine measurement of $m$ quantities $A_{1}, \ldots, A_{m}$. Note also the following: If $\left\{\mathcal{H}_{\alpha}, \lambda_{\alpha}, R_{\alpha}\right\}$ is a strong formal measurement of $A=\left(A_{1}, \ldots, A_{m}\right)$, then $\left\{\mathcal{H}_{\alpha}, \lambda_{\alpha}^{(i)}, R_{\alpha}\right\}$ is a strong formal measurement of $A_{i}$, but if $\left\{\mathcal{H}_{\alpha}, \lambda_{\alpha}, R_{\alpha}\right\}$ is an ideal, resp. normal, resp. standard, measurement of $A,\left\{\mathcal{H}_{\alpha}, \lambda_{\alpha}^{(i)}, R_{\alpha}\right\}$ need not be ideal, resp. normal, resp. standard.

There is a crucial point to observe: the same operator may belong to different commuting families. Consider, for example, a measurement of $A=\left(A_{1}, \ldots, A_{m}\right)$ and one of $B=\left(B_{1}, \ldots, B_{m}\right)$, where $A_{1}=B_{1} \equiv C$. Then while both measurements provide a measurement of $C$, they could be totally different: the operators $A_{i}$ and $B_{i}$ for $i \neq 1$ need not commute and the PVMs of $A$ and $B$, as well as any corresponding experiments $\mathscr{E}_{A}$ and $\mathscr{E}_{B}$, will be in general essentially different.

To emphasize this point we shall recall a famous example, the EPRB experiment [32, 14]: A pair of spin one-half particles, prepared in a spin-singlet state

$$
\psi=\frac{1}{\sqrt{2}}\left(\psi^{(+)} \otimes \psi^{(-)}+\psi^{(-)} \otimes \psi^{(+)}\right),
$$

are moving freely in opposite directions. Measurements are made, say by Stern-Gerlach magnets, on selected components of the spins of the two particles. Let $\mathbf{a}, \mathbf{b}, \mathbf{c}$ be three different unit vectors in space, let $\boldsymbol{\sigma}_{1} \equiv \boldsymbol{\sigma} \otimes I$ and let $\boldsymbol{\sigma}_{2} \equiv I \otimes \boldsymbol{\sigma}$, where $\boldsymbol{\sigma}=\left(\sigma_{x}, \sigma_{y}, \sigma_{z}\right)$ are the Pauli matrices. Then we could measure the operator $\boldsymbol{\sigma}_{1} \cdot \mathbf{a}$ by measuring either of the commuting families $\left(\boldsymbol{\sigma}_{1} \cdot \mathbf{a}, \boldsymbol{\sigma}_{2} \cdot \mathbf{b}\right)$ and $\left(\boldsymbol{\sigma}_{1} \cdot \mathbf{a}, \boldsymbol{\sigma}_{2} \cdot \mathbf{c}\right)$. However these measurements are different, both as weak and as strong measurements, and of course as experiments. In Bohmian mechanics the result obtained at one place at any given time will in fact depend upon the choice of the measurement simultaneously performed at the other place (i.e., on whether the spin of the other particle is measured along $\mathbf{b}$ or along $\mathbf{c}$ ). However, the statistics of the results won't be affected by the choice of measurement at the other place because both choices yield measurements of the same operator and thus their results must have the same statistical distribution.

\subsection{Functions of Measurements}

One of the most common experimental procedures is to recalibrate the scale of an experiment $\mathscr{E}$ : if $Z$ is the original result and $f$ an appropriate function, recalibration by $f$ leads to $f(Z)$ as the new result. Thus $f(\mathscr{E})$ has an obvious meaning. Moreover, if $\mathscr{E} \mapsto A$ according to (2.37) then $\mu_{\psi}^{f(Z)}=\mu_{\psi}^{Z} \circ f^{-1}=\mu_{\psi}^{A} \circ f^{-1}$, and

$$
\mu_{\psi}^{A} \circ f^{-1}(d \lambda)=\left\langle\psi, P^{A}\left(f^{-1}(d \lambda)\right) \psi\right\rangle=\left\langle\psi, P^{f(A)}(d \lambda) \psi\right\rangle
$$

where the last equality follows from the very definition of

$$
f(A)=\int f(\lambda) P^{A}(d \lambda)=\int \lambda P^{A}\left(f^{-1}(d \lambda)\right)
$$


provided by the spectral theorem. Thus,

$$
\text { if } \quad \mu_{\psi}^{Z}=\mu_{\psi}^{A} \quad \text { then } \quad \mu_{\psi}^{f(Z)}=\mu_{\psi}^{f(A)},
$$

i.e.,

$$
\text { if } \quad \mathscr{E} \mapsto A \quad \text { then } \quad f(\mathscr{E}) \mapsto f(A) .
$$

The notion of function of a formal measurement has then an unequivocal meaning: if $\mathcal{M}$ is a weak formal measurement defined by the PVM $P$ then $f(\mathcal{M})$ is the weak formal measurement defined by the PVM $P \circ f^{-1}$, so that if $\mathcal{M}$ is a measurement of $A$ then $f(\mathcal{M})$ is a measurement of $f(A)$; for a strong formal measurement $\mathcal{M}=\left\{\mathcal{H}_{\alpha}, \lambda_{\alpha}, R_{\alpha}\right\}$ the self-evident requirement that the recalibration not affect the wave function transitions induced by $\mathcal{M}$ leads to $f(\mathcal{M})=\left\{\mathcal{H}_{\alpha}, f\left(\lambda_{\alpha}\right), R_{\alpha}\right\}$. Note that if $\mathcal{M}$ is a standard measurement, $f(\mathcal{M})$ will in general not be standard (since in general $f$ can be many-to-one).

To highlight some subtleties of the notion of function of measurement we shall discuss two examples: Suppose that $\mathcal{M}$ and $\mathcal{M}^{\prime}$ are respectively measurements of the commuting families $A=\left(A_{1}, A_{2}\right)$ and $B=\left(B_{1}, B_{2}\right)$, with $A_{1} A_{2}=B_{1} B_{2}=C$. Let $f: \mathbb{R}^{2} \rightarrow \mathbb{R}, f\left(\lambda_{1}, \lambda_{2}\right)=\lambda_{1} \lambda_{2}$. Then both $f(\mathcal{M})$ and $f\left(\mathcal{M}^{\prime}\right)$ are measurement of the same self-adjoint operator $C$. Nevertheless, as strong measurements or as experiments, they could be very different: if $A_{2}$ and $B_{2}$ do not commute they will be associated with different families of spectral projections. (Even more simply, consider measurements $\mathcal{M}_{x}$ and $\mathcal{M}_{y}$ of $\sigma_{x}$ and $\sigma_{y}$ and let $f(\lambda)=\lambda^{2}$. Then $f\left(\mathcal{M}_{x}\right)$ and $f\left(\mathcal{M}_{y}\right)$ are measurement of $I$ - so that the result must be 1 ) - but the two strong measurements, as well as the corresponding experiments, are completely different.)

The second example is provided by measurements designed to determine whether the operator $A=\sum \lambda_{\alpha} P_{\mathcal{H}_{\alpha}}$ (the $\lambda_{\alpha}$ 's are distinct) has values in some given set $\Delta$. This determination can be accomplished in at least two different ways: Suppose that $\mathcal{M}$ is an ideal measurement of $A$ and let $1_{\Delta}(\lambda)$ be the characteristic function of the set $\Delta$. Then we could perform $1_{\Delta}(\mathcal{M})$, that is, we measure $A$ and see whether " $A \in \Delta$ ". But we could also perform an "ideal determination of $A \in \Delta^{\prime}$, that is, an ideal measurement of $1_{\Delta}(A)=P^{A}(\Delta)$. Now, both measurements provide a "measurement of $A \in \Delta$ " (i.e., of the operator $1_{\Delta}(A)$ ), since in both cases the results 1 and 0 get assigned the same probabilities. However, as strong measurements, they are different: when $1_{\Delta}(\mathcal{M})$ is performed, and the result 1 is obtained, $\psi$ undergoes the transition

$$
\psi \rightarrow P_{\mathcal{H}_{\alpha}} \psi
$$

where $\alpha$ is the outcome with $\lambda_{\alpha} \in \Delta$ that actually occurs. On the other hand, for an ideal measurement of $1_{\Delta}(A)$, the occurrence of the result 1 will generate the transition

$$
\psi \rightarrow P^{A}(\Delta) \psi=\sum_{\lambda_{\alpha} \in \Delta} P_{\mathcal{H}_{\alpha}} \psi
$$

Note that in this case the state of the system is changed as little as possible. For example, suppose that two eigenvalues, say $\lambda_{\alpha_{1}}, \lambda_{\alpha_{2}}$, belong to $\Delta$ and $\psi=\psi_{\alpha_{1}}+\psi_{\alpha_{2}}$; then determination by performing $1_{\Delta}(\mathcal{M})$ will lead to either $\psi_{\alpha_{1}}$ or $\psi_{\alpha_{2}}$, while the ideal determination of $A \in \Delta$ will not change the state. 


\subsection{Measurements of Operators with Continuous Spectrum}

We shall now reconsider the status of measurements of self-adjoint operators with continuous spectrum. First of all, we remark that while on the weak level such measurements arise very naturally - and, as already stressed in Section 3.1, are indeed the first to appear in Bohmian mechanics - there is no straightforward extension of the notion of strong measurement to operators with continuous spectrum.

However, for given set of real numbers $\Delta$, one may consider any determination of $A \in \Delta$, that is, any strong measurement of the spectral projection $P^{A}(\Delta)$. More generally, for any choice of a simple function

$$
f(\lambda)=\sum_{i=1}^{N} c_{i} 1_{\Delta_{i}}(\lambda),
$$

one may consider the strong measurements of $f(A)$. In particular, let $\left\{f^{(n)}\right\}$ be a sequence of simple functions converging to the identity, so that $f^{(n)}(A) \rightarrow A$, and let $\mathcal{M}_{n}$ be measurements of $f^{(n)}(A)$. Then $\mathcal{M}_{n}$ are approximate measurements of $A$.

Observe that the foregoing applies to operators with discrete spectrum, as well as to operators with continuous spectrum. But note that while on the weak level we always have

$$
\mathcal{M}_{n} \rightarrow \mathcal{M}
$$

where $\mathcal{M}$ is a (general) weak measurement of $A$ (in the sense of (3.4)), if $A$ has continuous spectrum $\mathcal{M}$ will not exist as a strong measurement (in any reasonable generalized sense, since this would imply the existence of a bounded-operator-valued function $R_{\lambda}$ on the spectrum of $A$ such that $R_{\lambda}^{*} R_{\lambda} d \lambda=P^{A}(d \lambda)$, which is clearly impossible). In other words, in this case there can be no actual (generalized) strong measurement that the approximate measurements $\mathcal{M}_{n}$ approximate - which is perfectly reasonable.

\subsection{Sequential Measurements}

Suppose that $n$ measurements (with for each $i$, the $\lambda_{\alpha_{i}}^{(i)}$ distinct)

$$
\mathcal{M}_{1} \equiv\left\{\mathcal{H}_{\alpha_{1}}^{(1)}, \lambda_{\alpha_{1}}^{(1)}, R_{\alpha_{1}}^{(1)}\right\}, \ldots, \mathcal{M}_{n} \equiv\left\{\mathcal{H}_{\alpha_{n}}^{(n)}, \lambda_{\alpha_{n}}^{(n)}, R_{\alpha_{n}}^{(n)}\right\}
$$

of operators (which need not commute)

$$
A_{1}=\sum_{\alpha_{1}} \lambda_{\alpha_{1}}^{(1)} P_{\alpha_{1}}^{(1)}, \ldots, A_{n}=\sum_{\alpha_{n}} \lambda_{\alpha_{n}}^{(n)} P_{\alpha_{n}}^{(n)}
$$

are successively performed on our system at times $0<t_{1}<t_{2}<\cdots<t_{N}$. Assume that the duration of any single measurement is small with respect to the time differences $t_{i}-t_{i-1}$, so that the measurements can be regarded as instantaneous. If in between two successive measurements the system's wave function changes unitarily with the operators $U_{t}$ then, using obvious notation,

$$
\operatorname{Prob}_{\psi}\left(A_{1}=\lambda_{\alpha_{1}}^{(1)}, \ldots, A_{n}=\lambda_{\alpha_{n}}^{(n)}\right)=\left\|R_{\alpha_{n}}^{(n)}\left(t_{n}\right) \cdots R_{\alpha_{1}}^{(1)}\left(t_{1}\right) \psi\right\|^{2},
$$

where $R_{\alpha_{i}}^{(i)}(t)=U_{t}^{-1} R_{\alpha_{i}}^{(i)} U_{t}$ and $\psi$ is the initial $(t=0)$ wave function. 
To understand how (3.19) comes about consider first the case where $n=2$ and $t_{2} \approx t_{1} \approx 0$. According to standard probability rules, the probability of obtaining the results $Z_{1}=\lambda_{\alpha_{1}}^{(1)}$ for the first measurement and $Z_{2}=\lambda_{\alpha_{2}}^{(2)}$ for the second one is the product ${ }^{19}$

$$
\operatorname{Prob}_{\psi}\left(Z_{2}=\lambda_{\alpha_{2}}^{(2)} \mid Z_{1}=\lambda_{\alpha_{1}}^{(1)}\right) \cdot \operatorname{Prob}_{\psi}\left(Z_{1}=\lambda_{\alpha_{1}}^{(1)}\right)
$$

where the first term is the probability of obtaining $\lambda_{\alpha_{2}}^{(2)}$ given that the result of the first measurement is $\lambda_{\alpha_{1}}^{(1)}$. Since $\mathcal{M}_{1}$ then transforms the wave function $\psi$ to $R_{\alpha_{1}}^{(1)} \psi$, the (normalized) initial wave function for $\mathcal{M}_{2}$ is $R_{\alpha_{1}}^{(1)} \psi /\left\|R_{\alpha_{1}}^{(1)} \psi\right\|$, this probability is equal to

$$
\frac{\left\|R_{\alpha_{2}}^{(2)} R_{\alpha_{1}}^{(1)} \psi\right\|^{2}}{\left\|R_{\alpha_{1}}^{(1)} \psi\right\|^{2}} .
$$

The second term, the probability of obtaining $\lambda_{\alpha_{1}}^{(1)}$, is of course $\left\|R_{\alpha_{1}}^{(1)} \psi\right\|^{2}$. Thus

$$
\operatorname{Prob}_{\psi}\left(A^{(1)}=\lambda_{\alpha_{1}}^{(1)}, A^{(2)}=\lambda_{\alpha_{2}}^{(2)}\right)=\left\|R_{\alpha_{2}}^{(2)} R_{\alpha_{1}}^{(1)} \psi\right\|^{2}
$$

in this case. Note that, in agreement with the analysis of discrete experiments (see Eq. (2.20)), the probability of obtaining the results $\lambda_{\alpha_{1}}^{(1)}$ and $\lambda_{\alpha_{2}}^{(2)}$ turns out to be the square of the norm of the final system wave function associated with these results. Now, for general times $t_{1}$ and $t_{2}-t_{1}$ between the preparation of $\psi$ at $t=0$ and the performance of $\mathcal{M}_{1}$ and between $\mathcal{M}_{1}$ and $\mathcal{M}_{2}$, respectively, the final system wave function is $R_{\alpha_{2}}^{(2)} U_{t_{2}-t_{1}} R_{\alpha_{1}}^{(1)} U_{t_{1}} \psi=R_{\alpha_{2}}^{(2)} U_{t_{2}} U_{t_{1}}^{-1} R_{\alpha_{1}}^{(1)} U_{t_{1}} \psi$. But $\left\|R_{\alpha_{2}}^{(2)} U_{t_{2}} U_{t_{1}}^{-1} R_{\alpha_{1}}^{(1)} U_{t_{1}} \psi\right\|=\left\|U_{t_{2}}^{-1} R_{\alpha_{2}}^{(2)} U_{t_{2}} U_{t_{1}}^{-1} R_{\alpha_{1}}^{(1)} U_{t_{1}} \psi\right\|$, and it is easy to see, just as for the simple case just considered, that the square of the latter is the probability for the corresponding result, whence (3.19) for $n=2$. Iterating, i.e., by induction, we arrive at (3.19) for general $n$.

We note that when the measurements $\mathcal{M}_{1}, \ldots \mathcal{M}_{n}$ are ideal, the operators $R_{\alpha_{i}}^{(i)}$ are the orthogonal projections $P_{\alpha_{i}}^{(i)}$, and equation (3.19) becomes the standard formula for the joint probabilities of the results of a sequence of measurements of quantum observables, usually known as Wigner's formula [76].

It is important to observe that, even for ideal measurements, the joint probabilities given by (3.19) are not in general a consistent family of joint distributions: summation in (3.19) over the outcomes of the $i$-th measurement does not yield the joint probabilities for the results of the measurements of the operators $A_{1}, \ldots, A_{i-1}, A_{i+1}, \ldots A_{n}$ performed at the times $t_{1}, \ldots, t_{i-1}, t_{i+1}, \ldots t_{n}$. (By rewriting the right hand side of (3.19) as $\left\langle\psi, R_{\alpha_{1}}^{(1)}\left(t_{n}\right)^{*} \cdots R_{\alpha_{n}}^{(n)}\left(t_{n}\right)^{*} R_{\alpha_{n}}^{(n)}\left(t_{n}\right) R_{\alpha_{1}}^{(1)}\left(t_{1}\right) \psi\right\rangle$ one easily sees that the "sum rule" will be satisfied when $i=n$ or if the operators $R_{\alpha_{i}}^{(i)}\left(t_{i}\right)$ commute. More generally, the consistency is guaranteed by the "decoherence conditions" of Griffiths, Omnès, Gell-Mann and Hartle, and Goldstein and Page [46, 35, 44].

\footnotetext{
${ }^{19}$ This is so because of the conditional independence of the outcomes of two successive measurements given the final conditional wave function for the first measurement. More generally, the outcome of any measurement depends only on the wave function resulting from the preceding one. For Bohmian experiments this independence is a direct consequence of (2.10). One may wonder about the status of this independence for orthodox quantum theory. We stress that while this issue might be problematical for orthodox quantum theory, it is not a problem for Bohmian mechanics: the conditional independence of two successive measurements is a consequence of the theory. (For more on this point, see [25]).) We also would like to stress that this independence assumption is in fact crucial for orthodox quantum theory. Without it, it is hard to see how one could ever be justified in invoking the quantum formalism. Any measurement we may consider will follow many earlier measurements.
} 
This failure of consistency means that the marginals of the joint probabilities given by (3.19) are not themselves given by the corresponding case of the formula. This should, however, come as no surprise: Since performing the measurement $\mathcal{M}_{i}$ affects the state of the system, the outcome of $\mathcal{M}_{i+1}$ should in general depend on whether or not $\mathcal{M}_{i}$ has been performed. Note that there is nothing particularly quantum in the fact that measurements matter in this way: They matter even for genuine measurements (unlike those we have been considering, in which nothing need be genuinely measured), and even in classical physics, if the measurements are such that they affect the state of the system.

The sequences of results $\lambda_{\alpha} \equiv\left(\lambda_{\alpha_{1}}^{(1)}, \ldots, \lambda_{\alpha_{n}}^{(n)}\right)$, the associated state transformations $R_{\alpha} \equiv$ $R_{\alpha_{n}}^{(n)} U_{t_{n}-t_{n-1}} R_{\alpha_{n-1}}^{(n-1)} \cdots R_{\alpha_{1}}^{(1)} U_{t_{1}}$, and the probabilities (3.19) (i.e., given by $p_{\alpha}=\left\|R_{\alpha}\right\|^{2}$ ) define what we shall call a sequential measurement of $\mathcal{M}_{1}, \cdots \mathcal{M}_{n}$, which we shall denote by $\mathcal{M}_{n} \otimes \ldots \otimes \mathcal{M}_{1}$. A sequential measurement does not in general define a formal measurement, neither weak nor strong, since $R_{\alpha}^{*} R_{\alpha}$ need not be a projection. This fact might seem disturbing (see, e.g., 21]); we shall take up this issue in the next section.

\subsection{Some Summarizing Remarks}

The notion of formal measurement we have explored in this section is at the heart of the quantum formalism. It embodies the two essential ingredients of a quantum measurement: the self-adjoint operator $A$ which represents the measured observable and the set of state transformations $R_{\alpha}$ associated with the measured results. The operator always carries the information about the statistics of possible results. The state transformations prescribe how the state of the system changes when the measurement is performed. For ideal measurement the latter information is also provided by the operator, but in general additional structure (the $R_{\alpha}$ 's) is required.

There are some important morals to draw. The association between measurements and operators is many-to-one: the same operator $A$ can be measured by many different measurements, for example ideal, or normal but not ideal. Among the possible measurements of $A$, we must consider all possible measurements of commuting families of operators that include $A$, each of which may correspond to entirely different experimental setups.

A related fact: not all measurements are ideal measurements. ${ }^{20}$ No argument, physical or mathematical, suggests that ideal measurements should be regarded as "more correct" than any

\footnotetext{
${ }^{20}$ In this regard we observe that the vague belief in a universal collapse rule is as old, almost, as quantum mechanics. It is reflected in von Neumann's formulation of quantum mechanics [74, based on two distinct dynamical laws: a unitary evolution between measurements, and a nonunitary evolution when measurements are performed. However, von Neumann's original proposal [74] for the nonunitary evolution-that when a measurement of $A=\sum_{\alpha} \lambda_{\alpha} P_{\mathcal{H}_{\alpha}}$ is performed upon a system in the state given by the density matrix $W$, the state of the system after the measurement is represented by the density matrix

$$
W^{\prime}=\sum_{\alpha} \sum_{\beta}\left\langle\phi_{\alpha \beta}, W \phi_{\alpha \beta}\right\rangle P_{\left[\phi_{\alpha \beta}\right]}
$$

where, for each $\alpha,\left\{\phi_{\alpha \beta}\right\}$ is a basis for $\mathcal{H}_{\alpha}$ - does not treat the general measurement as ideal. Moreover, this expression in general depends on the choice of the basis $\left\{\phi_{\alpha \beta}\right\}$, and was thus criticized by Lüders 61 , who proposed the transformation

$$
W \rightarrow W^{\prime}=\sum_{\alpha} P_{\mathcal{H}_{\alpha}} W P_{\mathcal{H}_{\alpha}},
$$

as it gives a unique prescription. Note that for $W=P_{[\psi]}$, where $P_{[\psi]}$ is the projection onto the initial pure state $\psi, W^{\prime}=\sum_{\alpha} p_{\alpha} P_{\left[\psi_{\alpha}\right]}$, where $p_{\alpha}=\left|\left\langle\psi, P_{\mathcal{H}_{\alpha}} \psi\right\rangle\right|^{2}$ and $\psi_{\alpha}=P_{\mathcal{H}_{\alpha}} \psi$, corresponding to an ideal measurement.
} 
other type. In particular, the Wigner formula for the statistics of a sequence of ideal measurements is no more correct than the formula (3.19) for a sequence of more general measurement. Granting a privileged status to ideal measurements amounts to a drastic and arbitrary restriction on the quantum formalism qua measurement formalism, since many (in fact most) real world measurements would be left out.

In this regard we note that the arbitrary restriction to ideal measurements affects the research program of "decoherent" or "consistent" histories [35, 65, 46, since Wigner's formula for a sequence of ideal measurements is unquestionably at its basis. (It should be emphasized however that the special status granted to ideal measurements is probably not the main difficulty with this approach. The no-hidden-variables theorems, which we shall discuss in Section 7 , show that the totality of different families of weakly decohering histories, with their respective probability formulas, is genuinely inconsistent. While such inconsistency is perfectly acceptable for a measurement formalism, it is hard to see how it can be tolerated as the basis of what is claimed to be a fundamental theory. For more on this, see [25, 43].

\section{The Extended Quantum Formalism}

As indicated in Section 2.9, the textbook quantum formalism is merely an idealization. As just stressed, not all real world measurements are ideal. In fact, in the real world the projection postulate - that when the measurement of an observable yields a specific value, the wave function of the system is replaced by its projection onto the corresponding eigenspace - is rarely obeyed. More importantly, a great many significant real-world experiments are simply not at all associated with operators in the usual way. Consider for example an electron with fairly general initial wave function, and surround the electron with a "photographic" plate, away from (the support of the wave function of) the electron, but not too far away. This setup measures the position of "escape" of the electron from the region surrounded by the plate. Notice that since in general the time of escape is random, it is not at all clear which operator should correspond to the escape positionit should not be the Heisenberg position operator at a specific time, and a Heisenberg position operator at a random time has no meaning. In fact, there is presumably no such operator, so that for the experiment just described the probabilities for the possible results cannot be expressed in the form (2.37), and in fact are not given by the spectral measure for any operator.

Time measurements, for example escape times or decay times, are particularly embarrassing for the quantum formalism. This subject remains mired in controversy, with various research groups proposing their own favorite candidates for the "time operator" while paying little attention to the proposals of the other groups. For an analysis of time measurements within the framework of Bohmian mechanics, see [20]; in this regard see also [57, 58, 59, 47.

Because of these and other difficulties, it has been proposed that we should go beyond operators-as-observables, to "generalized observables," described by mathematical objects even more abstract than operators (see, e.g., the books of Davies [21, Holevo [50] and Kraus [55]). The basis of this generalization lies in the observation that, by the spectral theorem, the concept of self-adjoint operator is completely equivalent to that of (a normalized) projection-valued measure (PVM), an orthogonal-projection-valued additive set function, on the value space $\mathbb{R}$. Orthogonal projections are among the simplest examples of positive operators, and a natural generalization of a "quantum observable" is provided by a positive-operator-valued measure (POVM): a normalized, countably additive set function $O$ whose values are positive operators on a Hilbert space 
$\mathcal{H}$. When a POVM is sandwiched by a wave function it generates a probability distribution

$$
\mu_{\psi}^{O}: \Delta \mapsto \mu_{\psi}^{O}(\Delta) \equiv\langle\psi, O(\Delta) \psi\rangle
$$

in exactly the same manner as a PVM.

\subsection{POVMs and Bohmian Experiments}

From a fundamental perspective, it may seem that we would regard this generalization, to positive-operator-valued measures, as a step in the wrong direction, since it supplies us with a new, much larger class of fundamentally unneeded abstract mathematical entities far removed from the basic ingredients of Bohmian mechanics. However from the perspective of Bohmian phenomenology positive-operator-valued measures form an extremely natural class of objectsindeed more natural than projection-valued measures.

To see how this comes about observe that (2.18) defines a family of bounded linear operators $R_{\alpha}$ by

$$
P_{\left[\Phi_{\alpha}\right]}\left[U\left(\psi \otimes \Phi_{0}\right)\right]=\left(R_{\alpha} \psi\right) \otimes \Phi_{\alpha},
$$

in terms of which we may rewrite the probability (2.20) of obtaining the result $\lambda_{\alpha}$ (distinct) in a generic discrete experiment as

$$
p_{\alpha}=\left\|\psi_{\alpha}\right\|^{2}=\left\|R_{\alpha} \psi\right\|^{2}=\left\langle\psi, R_{\alpha}^{*} R_{\alpha} \psi\right\rangle .
$$

By the unitarity of the overall evolution of system and apparatus we have that $\sum_{\alpha}\left\|\psi_{\alpha}\right\|^{2}=$ $\sum_{\alpha}\left\langle\psi, R_{\alpha}^{*} R_{\alpha} \psi\right\rangle=1$ for all $\psi \in \mathcal{H}$, whence

$$
\sum_{\alpha} R_{\alpha}^{*} R_{\alpha}=I
$$

The operators $O_{\alpha} \equiv R_{\alpha}^{*} R_{\alpha}$ are obviously positive, i.e.,

$$
\left\langle\psi, O_{\alpha} \psi\right\rangle \geq 0 \quad \text { for all } \quad \psi \in \mathcal{H}
$$

and by (4.4) sum up to the identity,

$$
\sum_{\alpha} O_{\alpha}=I
$$

Thus we may associate with a generic discrete experiment $\mathscr{E}$ - with no assumptions about reproducibility or anything else, but merely unitarity - a POVM

$$
O(\Delta)=\sum_{\lambda_{\alpha} \in \Delta} O_{\alpha} \equiv \sum_{\lambda_{\alpha} \in \Delta} R_{\alpha}^{*} R_{\alpha}
$$

in terms of which the statistics of the results can be expressed in a compact way: the probability that the result of the experiment lies in a set $\Delta$ is given by

$$
\sum_{\lambda_{\alpha} \in \Delta} p_{\alpha}=\sum_{\lambda_{\alpha} \in \Delta}\left\langle\psi, O_{\alpha} \psi\right\rangle=\langle\psi, O(\Delta) \psi\rangle
$$

Moreover, it follows from (2.18) and (4.2) that $\mathscr{E}$ generates state transformations

$$
\psi \rightarrow \psi_{\alpha}=R_{\alpha} \psi
$$




\subsection{Formal Experiments}

The association between experiments and POVMs can be extended to a general experiment (2.34) in a straightforward way. In analogy with (2.37) we shall say that the POVM $O$ is associated with the experiment $\mathscr{E}$ whenever the probability distribution (2.35) of the results of $\mathscr{E}$ is equal to the probability measure (4.1) generated by $O$, i.e., ${ }^{21}$

$$
\mathscr{E} \mapsto O \quad \text { if and only if } \quad \rho_{\psi}^{Z}=\mu_{\psi}^{O},
$$

We may now proceed as in Section 3 and analyze on a formal level the association (4.10) by introducing the notions of weak and strong formal experiment as the obvious generalizations of (3.4) and (3.8):

Any positive-operator-valued measure $O$ defines the weak formal experiment $\mathcal{E} \equiv O$.

Any set $\left\{\lambda_{\alpha}\right\}$ of not necessarily distinct real numbers (or vectors of real numbers)

paired with any collection $\left\{R_{\alpha}\right\}$ of bounded operators on $\mathcal{H}$ such that $\sum R_{\alpha}^{*} R_{\alpha}=I$

defines the strong formal experiment $\mathcal{E} \equiv\left\{\lambda_{\alpha}, R_{\alpha}\right\}$ with associated POVM (4.7)

and state transformations 4.9.).

The notion of formal experiment is a genuine extension of that of formal measurement, the latter being the special case in which $O$ is a PVM and $R_{\alpha}^{*} R_{\alpha}$ are the projections.

Formal experiments share with formal measurements many features. This is so because all measure-theoretic properties of projection-valued measures extend to positive-operator-valued measures. For example, just as for PVMs, integration of real functions against positive-operatorvalued measure is a meaningful operation that generates self-adjoint operators: for given real (and measurable) function $f$, the operator $B=\int f(\lambda) O(d \lambda)$ is a self-adjoint operator defined, say, by its matrix elements $\langle\phi, B \psi\rangle=\int \lambda \mu_{\phi, \psi}(d \lambda)$ for all $\phi$ and $\psi$ in $\mathcal{H}$, where $\mu_{\phi, \psi}$ is the complex measure $\mu_{\phi, \psi}(d \lambda)=\langle\phi, O(d \lambda) \psi\rangle$. (We ignore the difficulties that might arise if $f$ is not bounded.) In particular, with $O$ is associated the self-adjoint operator

$$
A_{O} \equiv \int \lambda O(d \lambda)
$$

It is however important to observe that this association (unlike the case of PVMs, for which the spectral theorem provides the inverse) is not invertible, since the self-adjoint operator $A_{O}$ is always associated with the PVM provided by the spectral theorem. Thus, unlike PVMs, POVMs are not equivalent to self-adjoint operators. In general, the operator $A_{O}$ will carry information only about the mean value of the statistics of the results,

$$
\int \lambda\langle\psi, O(d \lambda) \psi\rangle=\left\langle\psi, A_{O} \psi\right\rangle
$$

while for the higher moments we should expect that

$$
\int \lambda^{n}\langle\psi, O(d \lambda) \psi\rangle \neq\left\langle\psi, A_{O}^{n} \psi\right\rangle
$$

\footnotetext{
${ }^{21}$ Whenever (4.10) is satisfied we may say that the experiment $\mathscr{E}$ is a measurement of the generalized observable $O$. We shall however avoid this terminology in connection with generalized observables; even when it is standard (so that we use it), i.e., when $O$ is a PVM and thus equivalent to a self-adjoint operator, it is in fact improper.
} 
unless $O$ is a PVM.

What we have just described is an important difference between general formal experiments and formal measurements. This and other differences originate from the fact that a POVM is a much weaker notion than a PVM. For example, a POVM $O$ on $\mathbb{R}^{m}$-like ordinary measures and unlike PVMs - need not be a product measure: If $O_{1}, \ldots, O_{m}$ are the marginals of $O$,

$$
O_{1}\left(\Delta_{1}\right)=O\left(\Delta_{1} \times \mathbb{R}^{m-1}\right), \ldots, O_{m}\left(\Delta_{m}\right)=O\left(\mathbb{R}^{m-1} \times \Delta_{m}\right),
$$

the product POVM $O_{1} \times \cdots \times O_{m}$ will be in general different from $O$. (This is trivial since any probability measure on $\mathbb{R}^{m}$ times the identity is a POVM.)

Another important difference between the notion of POVM and that of PVM is this: while the projections $P(\Delta)$ of a PVM, for different $\Delta$ 's, commute, the operators $O(\Delta)$ of a generic POVM need not commute. An illustration of how this may naturally arise is provided by sequential measurements.

A sequential measurement (see Section [3.9) $\mathcal{M}_{n} \otimes \ldots \otimes \mathcal{M}_{1}$ is indeed a very simple example of a formal experiment that in general is not a formal measurement (see also Davies [21]). We have that

$$
\mathcal{M}_{n} \otimes \ldots \otimes \mathcal{M}_{1}=\left\{\lambda_{\alpha}, R_{\alpha}\right\}
$$

where

$$
\lambda_{\alpha} \equiv\left(\lambda_{\alpha_{1}}^{(1)}, \ldots, \lambda_{\alpha_{n}}^{(n)}\right)
$$

and

$$
R_{\alpha} \equiv R_{\alpha_{n}}^{(n)} U_{t_{n}-t_{n-1}} R_{\alpha_{n-1}}^{(n-1)} \cdots R_{\alpha_{1}}^{(1)} \cdot U_{t_{1}} .
$$

Note that since $p_{\alpha}=\left\|R_{\alpha} \psi\right\|^{2}$, we have that

$$
\sum_{\alpha} R_{\alpha}^{*} R_{\alpha}=I
$$

, which also follows directly using

$$
\sum_{\alpha_{j}} R_{\alpha_{j}}^{(j) *} R_{\alpha_{j}}^{(j)}=I, \quad j=1, \ldots, n
$$

Now, with $\mathcal{M}_{n} \otimes \ldots \otimes \mathcal{M}_{1}$ is associated the POVM

$$
O(\Delta)=\sum_{\lambda_{\alpha} \in \Delta} R_{\alpha}^{*} R_{\alpha} .
$$

Note that $O(\Delta)$ and $O\left(\Delta^{\prime}\right)$ in general don't commute since in general $R_{\alpha}$ and $R_{\beta}$ may fail to do so.

An interesting class of POVMs for which $O(\Delta)$ and $O\left(\Delta^{\prime}\right)$ do commute arises in association with the notion of an "approximate measurement" of a self-adjoint operator: suppose that the result $Z$ of a measurement $\mathcal{M}=P^{A}$ of a self-adjoint operator $A$ is distorted by the addition of an independent noise $N$ with symmetric probability distribution $\eta(\lambda)$. Then the result $Z+N$ of the experiment, for initial system wave function $\psi$, is distributed according to

$$
\Delta \mapsto \int_{\Delta} \int_{\mathbb{R}} \eta\left(\lambda-\lambda^{\prime}\right)\left\langle\psi, P_{A}\left(d \lambda^{\prime}\right) \psi\right\rangle d \lambda,
$$


which can be rewritten as

$$
\Delta \mapsto\left\langle\psi, \int_{\Delta} \eta(\lambda-A) d \lambda \psi\right\rangle .
$$

Thus the result $Z+N$ is governed by the POVM

$$
O(\Delta)=\int_{\Delta} \eta(\lambda-A) d \lambda .
$$

The formal experiment defined by this POVM can be regarded as providing an approximate measurement of $A$. For example, let

$$
\eta(\lambda)=\frac{1}{\sigma \sqrt{2 \pi}} e^{-\frac{\lambda^{2}}{2 \sigma^{2}}}
$$

Then for $\sigma \rightarrow 0$ the POVM (4.13) becomes the PVM of $A$ and the experiment becomes a measurement of $A$.

Concerning the POVM (4.13) we wish to make two remarks. The first is that the $O(\Delta)$ 's commute since they are all functions of $A$. The second is that this POVM has a continuous density, i.e.,

$$
O(d \lambda)=o(\lambda) d \lambda \quad \text { where } \quad o(\lambda)=\eta(\lambda-A) .
$$

This is another difference between POVMs and PVMs: like ordinary measures and unlike PVMs, POVMs may have a continuous density. The reason this is possible for POVMs is that, for a POVM $O$, unlike for a PVM, given $\psi \in H$, the vectors $O(\Delta) \psi$ and $O\left(\Delta^{\prime}\right) \psi$, for $\Delta$ and $\Delta^{\prime}$ disjoint and arbitrarily small, need not be orthogonal. Otherwise, no density $o(d \lambda)$ could exist, because this would imply that there is a continuous family $\{o(\lambda) \psi\}$ of orthogonal vectors in $\mathcal{H}$.

Finally, we observe that unlike strong measurements, the notion of strong formal experiment can be extended to POVM with continuous spectrum (see Section 3.8). One may in fact define a strong experiment by $\mathcal{E}=\left\{\lambda, R_{\lambda}\right\}$, where $\lambda \mapsto R_{\lambda}$ is a continuous bounded-operator-valued function such that $\int R_{\lambda}^{*} R_{\lambda} d \lambda=I$. Then the statistics for the results of such an experiment is governed by the POVM $O(d \lambda) \equiv R_{\lambda}^{*} R_{\lambda} d \lambda$. For example, let

$$
R_{\lambda}=\xi(\lambda-A) \quad \text { where } \quad \xi(\lambda)=\frac{1}{\sqrt{\sigma} \sqrt[4]{2 \pi}} e^{-\frac{\lambda^{2}}{4 \sigma^{2}}} .
$$

Then $O(d \lambda)=R_{\lambda}^{*} R_{\lambda} d \lambda$ is the POVM (4.13) with $\eta$ given by (4.14). We observe that the state transformations (cf. the definition (2.6) of the conditional wave function)

$$
\psi \rightarrow R_{\lambda} \psi=\frac{1}{\sqrt{\sigma} \sqrt[4]{2 \pi}} e^{-\frac{(\lambda-A)^{2}}{4 \sigma^{2}}} \psi
$$

can be regarded as arising from a von Neumann interaction with Hamiltonian (3.12) (and $\gamma T=1$ ) and ready state of the apparatus

$$
\Phi_{0}(y)=\frac{1}{\sqrt{\sigma} \sqrt[4]{2 \pi}} e^{-\frac{y^{2}}{4 \sigma^{2}}} .
$$

Experiments with state transformations (4.15), for large $\sigma$, have been considered by Aharonov and coworkers (see, e.g., Aharonov, Anandan, and Vaidman [1]) as providing "weak measurements" of operators. (The effect of the measurement on the state of the system is "small" if $\sigma$ is sufficiently large). This terminology notwithstanding, it is important to observe that such experiments are not measurements of $A$ in the sense we have discussed here. They give information about the average value of $A$, since $\int \lambda\left\langle\psi, R_{\lambda}^{*} R_{\lambda} \psi\right\rangle d \lambda=\langle\psi, A \psi\rangle$, but presumably none about its higher moments. 


\subsection{From Formal Experiments to Experiments}

Just as with a formal measurement (see Section [3.3), with a formal experiment $\mathcal{E} \equiv\left\{\lambda_{\alpha}, R_{\alpha}\right\}$, we may associate a discrete experiment $\mathscr{E}$. The unitary map (2.18) of $\mathscr{E}$ will be given again by (3.10), i.e.,

$$
U: \psi \otimes \Phi_{0} \mapsto \sum_{\alpha}\left(R_{\alpha} \psi\right) \otimes \Phi_{\alpha}
$$

but now $R_{\alpha}^{*} R_{\alpha}$ of course need not be projection. The unitarity of $U$ follows immediately from the orthonormality of the $\Phi_{\alpha}$ using $\sum R_{\alpha}^{*} R_{\alpha}=I$. (Note that with a weak formal experiment $\mathcal{E} \equiv O=\left\{O_{\alpha}\right\}$ we may associate many inequivalent discrete experiments, defined by (4.16) with operators $R_{\alpha} \equiv U_{\alpha} \sqrt{O_{\alpha}}$, for any choice of unitary operators $U_{\alpha}$.)

We shall now discuss a concrete example of a discrete experiment defined by a formal experiment which will allow us to make some more further comments on the issue of reproducibility discussed in Section 2.8 .

Let $\left\{\ldots, e_{-1}, e_{0}, e_{1}, \ldots\right\}$ be an orthonormal basis in the system Hilbert space $\mathcal{H}$, let $P_{-}, P_{0}, P_{+}$ be the orthogonal projections onto the subspaces $\widetilde{\mathcal{H}}_{-}, \mathcal{H}_{0}, \widetilde{\mathcal{H}}_{+}$spanned by $\{e\}_{\alpha<0},\left\{e_{0}\right\},\{e\}_{\alpha>0}$ respectively, and let $V_{+}, V_{-}$be the right and left shift operators,

$$
V_{+} e_{\alpha}=e_{\alpha+1}, \quad V_{-} e_{\alpha}=e_{\alpha-1}
$$

Consider the strong formal experiment $\mathcal{E}$ with the two possible results $\lambda_{ \pm}= \pm 1$ and associated state transformations

$$
R_{ \pm 1}=V_{ \pm}\left(P_{ \pm}+\frac{1}{\sqrt{2}} P_{0}\right)
$$

Then the unitary $U$ of the corresponding discrete experiment $\mathscr{E}$ is given by

$$
U: \psi \otimes \Phi_{0} \rightarrow R_{-} \psi \otimes \Phi_{-}+R_{+} \psi \otimes \Phi_{+},
$$

where $\Phi_{0}$ is the ready state of the apparatus and $\Phi_{ \pm}$are the apparatus states associated with the results \pm 1 . If we now consider the action of $U$ on the basis vectors $e_{\alpha}$,

$$
\begin{aligned}
& U\left(e_{\alpha} \otimes \Phi_{0}\right)=e_{\alpha+1} \otimes \Phi_{+} \quad \text { for } \alpha>0 \\
& U\left(e_{\alpha} \otimes \Phi_{0}\right)=e_{\alpha-1} \otimes \Phi_{-} \quad \text { for } \alpha<0 \\
& U\left(e_{0} \otimes \Phi_{0}\right)=\frac{1}{\sqrt{2}}\left(e_{1} \otimes \Phi_{+}+e_{-1} \otimes \Phi_{-}\right),
\end{aligned}
$$

we see immediately that

$$
U\left(\widetilde{\mathcal{H}}_{ \pm} \otimes \Phi_{0}\right) \subset \widetilde{\mathcal{H}}_{ \pm} \otimes \Phi_{ \pm 1}
$$

Thus (2.24) is satisfied and $\mathscr{E}$ is a reproducible experiment. Note however that the POVM $O=\left\{O_{-1}, O_{+1}\right\}$ associated with (4.17),

$$
O_{ \pm 1}=R_{ \pm 1}^{*} R_{ \pm 1}=P_{ \pm}+\frac{1}{2} P_{0}
$$

is not a PVM since the positive operators $O_{ \pm 1}$ are not projections, i.e, $O_{ \pm 1}^{2} \neq O_{ \pm 1}$. Thus $\mathscr{E}$ is not a measurement of any self-adjoint operator, which shows that without the assumption of the finite dimensionality of the subspaces $\widetilde{\mathcal{H}}_{\alpha}$ a reproducible discrete experiment need not be a measurement of a self-adjoint operator. 


\subsection{Measure-Valued Quadratic Maps}

We conclude this section with a remark about POVMs. Via (4.1) every POVM $O$ defines a "normalized quadratic map" from $\mathcal{H}$ to measures on some space (the value-space for the POVM). Moreover, every such map comes from a POVM in this way. Thus the two notions are equivalent:

4.1) defines a canonical one-to-one correspondence between POVMs and normalized measure-valued quadratic maps on $\mathcal{H}$.

To say that a measure-valued map on $\mathcal{H}$

$$
\psi \mapsto \mu_{\psi}
$$

is quadratic means that

$$
\mu_{\psi}=B(\psi, \psi)
$$

is the diagonal part of a sesquilinear map $B$, from $\mathcal{H} \times \mathcal{H}$ to the complex measures on some value space $\Lambda$. If $B(\psi, \psi)$ is a probability measure whenever $\|\psi\|=1$, we say that the map is normalized. ${ }^{22}$

Proposition (4.18) is a consequences of the following considerations: For a given POVM $O$ the map $\psi \mapsto \mu_{\psi}^{O}$, where $\mu_{\psi}^{O}(\Delta) \equiv\langle\psi, O(\Delta) \psi\rangle$, is manifestly quadratic, with $B(\phi, \psi)=\langle\phi, O(\cdot) \psi\rangle$, and it is obviously normalized. Conversely, let $\psi \mapsto \mu_{\psi}$ be a normalized measure-valued quadratic map, corresponding to some $B$, and write $B_{\Delta}(\phi, \psi)=B(\phi, \psi)[\Delta]$ for the complex measure $B$ at the Borel set $\Delta$. By the Schwartz inequality, applied to the positive form $B_{\Delta}(\phi, \psi)$, we have that $\left|B_{\Delta}(\phi, \psi)\right| \leq\|\psi\|\|\phi\|$. Thus, using Riesz's lemma [70], there is a unique bounded operator $O(\Delta)$ on $\mathcal{H}$ such that

$$
B_{\Delta}(\phi, \psi)=\langle\phi, O(\Delta) \psi\rangle
$$

Moreover, $O(\Delta)$, like $B_{\Delta}$, is countably additive in $\Delta$, and since $B(\psi, \psi)$ is a (positive) measure, $O$ is a positive-operator-valued measure, normalized because $B$ is.

A simple example of a normalized measure-valued quadratic map is

$$
\Psi \mapsto \rho^{\Psi}(d q)=|\Psi|^{2} d q
$$

whose associated POVM is the PVM $P^{\hat{Q}}$ for the position (configuration) operator

$$
\hat{Q} \Psi(q)=q \Psi(q) .
$$

Note also that if the quadratic map $\mu_{\psi}$ corresponds to the POVM $O$, then, for any unitary $U$, the composite map $\psi \mapsto \mu_{U \psi}$ corresponds to the POVM $U^{*} O U$, since $\langle U \psi, O(\Delta) U \psi\rangle=$ $\left\langle\psi, U^{*} O(\Delta) U \psi\right\rangle$. In particular for the map (4.21) and $U=U_{T}$, the composite map corresponds to the PVM $P^{\hat{Q}_{T}}$, with $\hat{Q}_{T}=U^{*} \hat{Q} U$, the Heisenberg position (configuration) at time $T$, since $U_{T}^{*} P^{\hat{Q}} U_{T}=P^{U_{T}^{*} \hat{Q} U_{T}}$.

${ }^{22} \mathrm{~A}$ sesquilinear map $B(\phi, \psi)$ is one that is linear in the second slot and conjugate linear in the first:

$$
\begin{aligned}
B\left(\phi, \alpha \psi_{1}+\beta \psi_{2}\right) & =\alpha B\left(\phi, \psi_{1}\right)+\beta B\left(\phi, \psi_{2}\right) \\
B\left(\alpha \phi_{1}+\beta \phi_{2}, \psi\right) & =\bar{\alpha} B\left(\phi_{1}, \psi\right)+\bar{\beta} B\left(\phi_{2}, \psi\right) .
\end{aligned}
$$

Clearly any such normalized $B$ can be chosen to be conjugate symmetric, $B(\psi, \phi)=\overline{B(\phi, \psi)}$, without affecting its diagonal, and it follows from polarization that any such $B$ must in fact be conjugate symmetric. 


\section{The General Emergence of Operators}

For Bohmian mechanics POVMs emerge naturally, not for discrete experiments, but for a general experiment (2.34). To see how this comes about consider the probability measure (2.35) giving the probability distribution of the result $Z=F\left(Q_{T}\right)$ of the experiment, where $Q_{T}$ is the final configuration of system and apparatus and $F$ is the calibration function expressing the numerical result, for example the orientation $\Theta$ of a pointer. Then the map

$$
\psi \mapsto \rho_{\psi}^{Z}=\rho_{\Psi_{T}} \circ F^{-1}
$$

from the initial wave function of the system to the probability distribution of the result, is quadratic since it arises from the sequence of maps

$$
\psi \mapsto \Psi=\psi \otimes \Phi_{0} \mapsto \Psi_{T}=U\left(\psi \otimes \Phi_{0}\right) \mapsto \rho_{\Psi_{T}}(d q)=\Psi_{T}^{*} \Psi_{T} d q \mapsto \rho_{\psi}^{Z}=\rho_{\Psi_{T}} \circ F^{-1}
$$

where the middle map, to the quantum equilibrium distribution, is obviously quadratic, while all the other maps are linear, all but the second trivially so. Now, by (4.18), the notion of such a quadratic map (5.1) is completely equivalent to that of a POVM on the system Hilbert space $\mathcal{H}$. (The sesquilinear map $B$ associated with (5.2) is $B\left(\psi_{1}, \psi_{2}\right)=\Psi_{1 T}^{*} \Psi_{2 T} d q \circ F^{-1}$, where $\Psi_{i T}=U\left(\psi_{i} \otimes \Phi_{0}\right)$.)

Thus the emergence and role of POVMs as generalized observables in Bohmian mechanics is merely an expression of the sesquilinearity of quantum equilibrium together with the linearity of the Schrödinger evolution. Thus the fact that with every experiment is associated a POVM, which forms a compact expression of the statistics for the possible results, is a near mathematical triviality. It is therefore rather dubious that the occurrence of POVMs - the simplest case of which is that of PVMs - as observables can be regarded as suggesting any deep truths about reality or about epistemology.

An explicit formula for the POVM defined by the quadratic map (5.1) follows immediately from (5.2):

$$
\rho_{\psi}^{Z}(d \lambda)=\left\langle\psi \otimes \Phi_{0}, U^{*} P^{\hat{Q}}\left(F^{-1}(d \lambda)\right) U \psi \otimes \Phi_{0}\right\rangle=\left\langle\psi \otimes \Phi_{0}, P_{0} U^{*} P^{\hat{Q}}\left(F^{-1}(d \lambda)\right) U P_{0} \psi \otimes \Phi_{0}\right\rangle
$$

where $P^{\hat{Q}}$ is the PVM for the position (configuration) operator (4.22) and $P_{0}$ is the projection onto $\mathcal{H} \otimes \Phi_{0}$, whence

$$
O(d \lambda)=1_{\Phi_{0}}^{-1} P_{0} U^{*} P^{\hat{Q}}\left(F^{-1}(d \lambda)\right) U P_{0} 1_{\Phi_{0}},
$$

where $1_{\Phi_{0}} \psi=\psi \otimes \Phi_{0}$ is the natural identification of $\mathcal{H}$ with $\mathcal{H} \otimes \Phi_{0}$. This is the obvious POVM reflecting the essential structure of the experiment. ${ }^{23}$

${ }^{23}$ This POVM can also be written as

$$
O(d \lambda)=\operatorname{tr}_{A}\left[P_{0} U^{*} P^{\hat{Q}}\left(F^{-1}(d \lambda)\right) U\right]
$$

where $\operatorname{tr}_{A}$ is the partial trace over the apparatus variables. The partial trace is a map $\operatorname{tr}_{A}: W \mapsto \operatorname{tr}_{A}(W)$, from trace class operators on the Hilbert space $\mathcal{H}_{S} \otimes \mathcal{H}_{A}$ to trace class operators on $\mathcal{H}_{S}$, uniquely defined by $\operatorname{tr}_{S}\left(\operatorname{tr}_{A}(W) B\right)=\operatorname{tr}_{S+A}(W B \otimes I)$, where $\operatorname{tr}_{S+A}$ and $\operatorname{tr}_{S}$ are the usual (scalar-valued) traces of operators on $\mathcal{H}_{S} \otimes \mathcal{H}_{A}$ and $\mathcal{H}_{S}$, respectively. For a trace class operator $B$ on $L^{2}(d x) \otimes L^{2}(d y)$ with kernel $B\left(x, y, x^{\prime}, y^{\prime}\right)$ we have $\operatorname{tr}_{A}(B)\left(x, x^{\prime}\right)=\int B\left(x, y, x^{\prime}, y\right) d y$. In (5.4) $\operatorname{tr}_{A}$ is applied to operators that need not be trace class-nor need the operator on the left be trace class - since, e.g., $O(\Lambda)=I$. The formula nonetheless makes sense. 
Note that the POVM (5.3) is unitarily equivalent to

$$
P_{0} P^{F\left(\hat{Q}_{T}\right)}(d \lambda) P_{0}
$$

where $\hat{Q}_{T}$ is the Heisenberg configuration of system and apparatus at time $T$. This POVM, acting on the subspace $\mathcal{H} \otimes \Phi_{0}$, is the projection to that subspace of a PVM, the spectral projections for $F\left(\hat{Q}_{T}\right)$. Naimark has shown (see, e.g., 21] ) that every POVM is equivalent to one that arises in this way, as the orthogonal projection of a PVM to a subspace. ${ }^{24}$

We shall now illustrate the association of POVMs with experiments by considering some special cases of (5.2).

\section{1 "No Interaction" Experiments}

Let $U=U_{S} \otimes U_{A}$ in (5.2) (hereafter the indices " $S$ " and " $A$ " shall refer, respectively, to system and apparatus). Then for $F(x, y)=y$ the measure-valued quadratic map defined by (5.2) is

$$
\psi \mapsto c(y)\|\psi\|^{2} d y
$$

where $c(y)=\left|U_{A} \Phi_{0}\right|^{2}(y)$, with POVM $O_{1}(d y)=c(y) d y I_{S}$, while for $F(q)=q=(x, y)$ the map is

$$
\psi \mapsto c(y)\left|U_{S} \psi\right|^{2}(x) d q
$$

with corresponding POVM $O_{2}(d q)=c(y) U_{S}^{*} P^{\hat{X}}(d x) U_{S} d y$. Neither $O_{1}$ nor $O_{2}$ is a PVM. However, if $F$ is independent of $y, F(x, y)=F(x)$, then the apparatus can be ignored in (5.2) or (5.3) and $O=U_{S}^{*} P^{\hat{X}} U_{S} \circ F^{-1}$, i.e.,

$$
O(d \lambda)=U_{S}^{*} P^{\hat{X}}\left(F^{-1}(d \lambda)\right) U_{S},
$$

which is manifestly a PVM-in fact corresponding to $F\left(\hat{X}_{T}\right)$, where $\hat{X}_{T}$ is the Heisenberg configuration of the system at the end of the experiment.

This case is somewhat degenerate: with no interaction between system and apparatus it hardly seems anything like a measurement. However, it does illustrate that it is "true" POVMs (i.e., those that aren't PVMs) that typically get associated with experiments-i.e., unless some special conditions hold (here that $F=F(x)$ ).

\section{2 "No $X "$ Experiments}

The map (5.2) is well defined even when the system (the $x$-system) has no translational degrees of freedom, so that there is no $x$ (or $X$ ). This will be the case, for example, when the system Hilbert space $\mathcal{H}_{S}$ corresponds to the spin degrees of freedom. Then $\mathcal{H}_{S}=\mathbb{C}^{n}$ is finite dimensional.

\footnotetext{
${ }^{24}$ If $O(d \lambda)$ is a POVM on $\Sigma$ acting on $\mathcal{H}$, then the Hilbert space on which the corresponding PVM acts is the natural Hilbert space associated with the data at hand, namely $L^{2}(\Sigma, \mathcal{H}, O(d \lambda))$, the space of $\mathcal{H}$-valued functions $\psi(\lambda)$ on $\Sigma$, with inner product given by $\int\langle\psi(\lambda), O(d \lambda) \phi(\lambda)\rangle$. (If this is not, in fact, positive definite, then the quotient with its kernel should be taken $-\psi(\lambda)$ should, in other words, be understood as the appropriate equivalence class.) Then $O(d \lambda)$ is equivalent to $P E(d \lambda) P$, where $E(\Delta)=\hat{1}_{\Delta}(\lambda)$, multiplication by $1_{\Delta}(\lambda)$, and $P$ is the orthogonal projection onto the subspace of constant $\mathcal{H}$-valued functions $\psi(\lambda)=\psi$.
} 
In such cases, the calibration $F$ of course is a function of $y$ alone, since there is no $x$. For $F=y$ the measure-valued quadratic map defined by (5.2) is

$$
\psi \mapsto\left|\left[U\left(\psi \otimes \Phi_{0}\right)\right](y)\right|^{2} d y,
$$

where $|\cdots|$ denotes the norm in $\mathbb{C}^{n}$.

This case is physically more interesting than the previous one, though it might appear rather puzzling since until now our measured systems have always involved configurations. After all, without configurations there is no Bohmian mechanics! However, what is relevant from a Bohmian perspective is that the composite of system and apparatus be governed by Bohmian mechanics, and this may well be the case if the apparatus has configurational degrees of freedom, even if what is called the system doesn't. Moreover, this case provides the prototype of many real-world experiments, e.g., spin measurements.

For the measurement of a spin component of a spin $-1 / 2$ particle - recall the description of the Stern-Gerlach experiment given in Section 2.5 - we let $\mathcal{H}_{S}=\mathbb{C}^{2}$, the spin space, with "apparatus" configuration $y=\mathbf{x}$, the position of the particle, and with suitable calibration $F(\mathbf{x})$. (For a real world experiment there would also have to be a genuine apparatus - a detector - that measures where the particle actually is at the end of the experiment, but this would not in any way affect our analysis. We shall elaborate upon this below.) The unitary $U$ of the experiment is the evolution operator up to time $T$ generated by the Pauli Hamiltonian (2.12), which under the assumption (2.14) becomes

$$
H=-\frac{\hbar^{2}}{2 m} \nabla^{2}-(b+a z) \sigma_{z}
$$

Moreover, as in Section 2.5, we shall assume that the initial particle wave function has the form $\Phi_{0}(\mathbf{x})=\Phi_{0}(z) \phi(x, y) .{ }^{25}$ Then for $F(\mathbf{x})=z$ the quadratic map (15.2) is

$$
\begin{aligned}
\psi & \mapsto\left(\left|\left\langle\psi^{+}, \psi\right\rangle\right|^{2}\left|\Phi_{T}^{(+)}(z)\right|^{2}+\left|\left\langle\psi^{-}, \psi\right\rangle\right|^{2}\left|\Phi_{T}^{(-)}(z)\right|^{2}\right) d z \\
& =\left\langle\psi, \mid \psi^{+}\right\rangle\left\langle\left.\psi^{+}|| \Phi_{T}^{(+)}(z)\right|^{2}+\mid \psi^{-}\right\rangle\left\langle\left.\psi^{-}|| \Phi_{T}^{(-)}(z)\right|^{2} \psi\right\rangle d z
\end{aligned}
$$

with POVM

$$
O(d z)=\left(\begin{array}{cc}
\left|\Phi_{T}^{(+)}(z)\right|^{2} & 0 \\
0 & \left|\Phi_{T}^{(-)}(z)\right|^{2}
\end{array}\right) d z
$$

where $\psi^{ \pm}$are the eigenvectors (2.13) of $\sigma_{z}$ and $\Phi_{T}^{( \pm)}$are the solutions of (2.15) computed at $t=T$, for initial conditions $\Phi_{0}{ }^{( \pm)}=\Phi_{0}(z)$.

Consider now the appropriate calibration for the Stern-Gerlach experiment, namely the function

$$
F(\mathbf{x})= \begin{cases}+1 & \text { if } z>0 \\ -1 & \text { if } z<0\end{cases}
$$

which assigns to the outcomes of the experiment the desired numerical results: if the particle goes up in the $z$-direction the spin is +1 , while if the particle goes down the spin is -1 . The corresponding POVM $O_{T}$ is defined by

$$
O_{T}(+1)=\left(\begin{array}{cc}
p_{T}^{+} & 0 \\
0 & p_{T}^{-}
\end{array}\right) \quad O_{T}(-1)=\left(\begin{array}{cc}
1-p_{T}^{+} & 0 \\
0 & 1-p_{T}^{-}
\end{array}\right)
$$

\footnotetext{
${ }^{25}$ We abuse notation here in using the notation $y=\mathbf{x}=(x, y, z)$. The $y$ on the right should of course not be confused with the one on the left.
} 
where

$$
p_{T}^{+}=\int_{0}^{\infty}\left|\Phi_{T}^{(+)}\right|^{2}(z) d z, \quad p_{T}^{-}=\int_{0}^{\infty}\left|\Phi_{T}^{(-)}\right|^{2}(z) d z .
$$

It should be noted that $O_{T}$ is not a PVM. However, as indicated in Section 2.5, as $T \rightarrow \infty$, $p_{T}^{+} \rightarrow 1$ and $p_{T}^{-} \rightarrow 0$, and the POVM $O_{T}$ becomes the PVM of the operator $\sigma_{z}$, i.e., $O_{T} \rightarrow P^{\sigma_{z}}$, defined by

$$
P(+1)=\left(\begin{array}{ll}
1 & 0 \\
0 & 0
\end{array}\right) \quad P(-1)=\left(\begin{array}{ll}
0 & 0 \\
0 & 1
\end{array}\right)
$$

and the experiment becomes a measurement of the operator $\sigma_{z}$.

\section{3 "No $Y "$ Experiments}

Suppose now that the "apparatus" involves no translational degrees of freedom, i.e., that there is no $y$ (or $Y$ ). For example, suppose the apparatus Hilbert space $\mathcal{H}_{A}$ corresponds to certain spin degrees of freedom, with $\mathcal{H}_{A}=\mathbb{C}^{n}$ finite dimensional. Then, of course, $F=F(x)$.

This case illustrates what measurements are not. If the apparatus has no configurational degrees of freedom, then neither in Bohmian mechanics nor in orthodox quantum mechanics is it a bona fide apparatus: Whatever virtues such an apparatus might otherwise have, it certainly can't generate any directly observable results (at least not when the system itself is microscopic). According to Bohr ([17], pages 73 and 90): "Every atomic phenomenon is closed in the sense that its observation is based on registrations obtained by means of suitable amplification devices with irreversible functioning such as, for example, permanent marks on the photographic plate" and "the quantum-mechanical formalism permits well-defined applications only to such closed phenomena." To stress this point, discussing particle detection Bell has said [7]: "Let us suppose that a discharged counter pops up a flag sayings 'Yes' just to emphasize that it is a macroscopically different thing from an undischarged counter, in a very different region of configuration space."

Experiments based on certain micro-apparatuses, e.g., "one-bit detectors" 73, provide a nice example of "No Y" experiments. We may think of a one-bit detector as a spin-1/2-like system (e.g., a two-level atom), with "down" state $\Phi_{0}$ (the ready state) and "up" state $\Phi_{1}$ and which is such that its configurational degrees of freedom can be ignored. Suppose that this "spin-system," in its "down" state, is placed in a small spatial region $\Delta_{1}$ and consider a particle whose wave function has been prepared in such a way that at $t=0$ it has the form $\psi=\psi_{1}+\psi_{2}$, where $\psi_{1}$ is supported by $\Delta_{1}$ and $\psi_{2}$ by $\Delta_{2}$ disjoint from $\Delta_{1}$. Assume that the particle interacts locally with the spin-system, in the sense that were $\psi=\psi_{1}$ the "spin" would flip to the "up" state, while were $\psi=\psi_{2}$ it would remain in its "down" state, and that the interaction time is negligibly small, so that other contributions to the Hamiltonian can be ignored. Then the initial state $\psi \otimes \Phi_{0}$ undergoes the unitary transformation

$$
U: \psi \otimes \Phi_{0} \rightarrow \Psi=\psi_{1} \otimes \Phi_{1}+\psi_{2} \otimes \Phi_{0}
$$

We may now ask whether $U$ defines an experiment genuinely measuring whether the particle is in $\Delta_{1}$ or $\Delta_{2}$. The answer of course is no (since in this experiment there is no apparatus property at all with which the position of the particle could be correlated) unless the experiment is (quickly) completed by a measurement of the "spin" by means of another (macroscopic) apparatus. In other words, we may conclude that the particle is in $\Delta_{1}$ only if the spin-system in effect pops up a flag saying "up". 


\section{4 "No $Y$ no $\Phi "$ Experiments}

Suppose there is no apparatus at all: no apparatus configuration $y$ nor Hilbert space $\mathcal{H}_{A}$, or, what amounts to the same thing, $\mathcal{H}_{A}=\mathbb{C}$. For calibration $F=x$ the measure-valued quadratic map defined by (5.2) is

$$
\psi \mapsto|U \psi(x)|^{2},
$$

with POVM $U^{*} P^{\hat{X}} U$, while the POVM for general calibration $F(x)$ is

$$
O(d \lambda)=U^{*} P^{\hat{X}}\left(F^{-1}(d \lambda)\right) U
$$

$O$ is a PVM, as mentioned in Section [5.1, corresponding to the operator $U^{*} F(\hat{X}) U=F\left(\hat{X}_{T}\right)$, where $\hat{X}_{T}$ is the Heisenberg position (configuration) operator at time $T$.

It is important to observe that even though these experiments suffer from the defect that no correlation is established between the system and an apparatus, this can easily be remedied-by adding a final detection measurement that measures the final actual configuration $X_{T}$-without in any way affecting the essential formal structure of the experiment. For these experiments the apparatus thus does not introduce any additional randomness, but merely reflects what was already present in $X_{T}$. All randomness in the final result

$$
Z=F\left(X_{T}\right)
$$

arises from randomness in the initial configuration of the system. ${ }^{26}$

For $F=x$ and $U=I$ the quadratic map is $\psi \mapsto|\psi(x)|^{2}$ with PVM $P^{\hat{X}}$, so that this (trivial) experiment corresponds to the simplest and most basic operator of quantum mechanics: the position operator. How other basic operators arise from experiments is what we are going to discuss next.

\subsection{The Basic Operators of Quantum Mechanics}

According to Bohmian mechanics, a particle whose wave function is real (up to a global phase), for example an electron in the ground state of an atom, has vanishing velocity, even though the quantum formalism assigns a nontrivial probability distribution to its momentum. It might thus seem that we are faced with a conflict between the predictions of Bohmian mechanics and those of the quantum formalism. This, however, is not so. The quantum predictions about momentum concern the results of an experiment that happens to be called a momentum measurement and a conflict with Bohmian mechanics with regard to momentum must reflect disagreement about the results of such an experiment.

One may base such an experiment on free motion followed by a final measurement of position. ${ }^{27}$ Consider a particle of mass $m$ whose wave function at $t=0$ is $\psi=\psi(\mathbf{x})$. Suppose no

\footnotetext{
${ }^{26}$ Though passive, the apparatus here plays an important role in recording the final configuration of the system. However, for experiments involving detections at different times, the apparatus plays an active role: Consider such an experiment, with detections at times $t_{1}, \ldots, t_{n}$, and final result $Z=F\left(X_{t_{1}}, \ldots, X_{t_{n}}\right)$. Though the apparatus introduces no extra randomness, it plays an essential role by changing the wave function of the system at the times $t_{1}, \ldots, t_{n}$ and thus changing the evolution of its configuration. These changes are reflected in the POVM structure that governs the statistical distribution of $Z$ for such experiments (see Section 3.9).

${ }^{27}$ The emergence of the momentum operator in such so-called time-of-flight measurements was discussed by Bohm in his 1952 article [15]. A similar derivation of the momentum operator can be found in Feynman and Hibbs 34.
} 
forces are present, that is, that all the potentials acting on the particle are turned off, and let the particle evolve freely. Then we measure the position $\mathbf{X}_{T}$ that it has reached at the time $t=T$. It is natural to regard $\mathbf{V}_{T}=\mathbf{X}_{T} / T$ and $\mathbf{P}_{T}=m \mathbf{X}_{T} / T$ as providing, for large $T$, approximations to the asymptotic velocity and momentum of the particle. It turns out that the probability distribution of $\mathbf{P}_{T}$, in the limit $T \rightarrow \infty$, is exactly what quantum mechanics prescribes for the momentum, namely $|\tilde{\psi}(\mathbf{p})|^{2}$, where

$$
\tilde{\psi}(\mathbf{p})=(\mathcal{F} \psi)(\mathbf{p})=\frac{1}{\sqrt{(2 \pi \hbar)^{3}}} \int e^{-\frac{i}{\hbar} \mathbf{p} \cdot \mathbf{x}} \psi(\mathbf{x}) d \mathbf{x}
$$

is the Fourier transform of $\psi$.

This result can be easily understood: Observe that $\left|\psi_{T}(\mathbf{x})\right|^{2} d \mathbf{x}$, the probability distribution of $\mathbf{X}_{T}$, is the spectral measure $\mu_{\psi}^{\hat{\mathbf{X}}_{T}}(d \mathbf{x})=\left\langle\psi, P^{\hat{\mathbf{X}}_{T}}(d \mathbf{x}) \psi\right\rangle$ of $\hat{\mathbf{X}}_{T}=U_{T}^{*} \hat{\mathbf{X}} U_{T}$, the (Heisenberg) position operator at time $t=T$; here $U_{t}$ is the free evolution operator and $\hat{\mathbf{X}}$ is, as usual, the position operator at time $t=0$. By elementary quantum mechanics (specifically, the Heisenberg equations of motion), $\hat{\mathbf{X}}_{T}=\frac{1}{m} \hat{\mathbf{P}} T+\hat{\mathbf{X}}$, where $\hat{\mathbf{P}} \equiv-i \hbar \nabla$ is the momentum operator. Thus as $T \rightarrow \infty$ the operator $m \hat{\mathbf{X}}_{T} / T$ converges to the momentum operator $\hat{\mathbf{P}}$, since $\hat{\mathbf{X}} / T$ is $O(1 / T)$, and the distribution of the random variable $\mathbf{P}_{T}$ accordingly converges to the spectral measure of $\hat{\mathbf{P}}$, given by $|\tilde{\psi}(\mathbf{p})|^{2}{ }^{28}$

The momentum operator arises from a $(T \rightarrow \infty)$ limit of "no $Y$ no $\Phi$ " single-particle experiments, each experiment being defined by the unitary operator $U_{T}$ (the free particle evolution operator up to time $T$ ) and calibration $F_{T}(\mathbf{x})=m \mathbf{x} / T$. Other standard quantum-mechanical operators emerge in a similar manner, i.e., from a $T \rightarrow \infty$ limit of appropriate single-particle experiments.

This is the case, for example, for the spin operator $\sigma_{z}$. As in Section [5.2, consider the evolution operator $U_{T}$ generated by Hamiltonian (5.7), but instead of (5.9), consider the calibration $F_{T}(\mathbf{x})=2 m z / a T^{2}$. This calibration is suggested by (2.16), as well as by the explicit form of the $z$-component of the position operator at time $t=T$,

$$
\hat{Z}_{T}=U_{T}^{*} \hat{Z} U_{T}=\hat{Z}+\frac{\hat{P}_{z}}{m} T+\frac{a}{2 m} \sigma_{z} T^{2}
$$

which follows from the Heisenberg equations

$$
m \frac{d^{2} \hat{Z}_{t}}{d t^{2}}=a \sigma_{z},\left.\quad \frac{d \hat{Z}_{t}}{d t}\right|_{t=0}=\hat{P}_{z} \equiv-i \hbar \frac{\partial}{\partial z}, \quad \hat{Z}_{0}=\hat{Z} .
$$

\footnotetext{
28 This formal argument can be turned into a rigorous proof by considering the limit of the characteristic function of $\mathbf{P}_{T}$, namely of the function $f_{T}(\boldsymbol{\lambda})=\int e^{i \boldsymbol{\lambda} \cdot \mathbf{p}} \rho_{T}(d \mathbf{p})$, where $\rho_{T}$ is the distribution of $m \mathbf{X}_{T} / T: f_{T}(\boldsymbol{\lambda})=$ $\left\langle\psi, \exp \left(i \boldsymbol{\lambda} \cdot m \hat{\mathbf{X}}_{T} / T\right) \psi\right\rangle$, and using the dominated convergence theorem 70 . this converges as $T \rightarrow \infty$ to $f(\boldsymbol{\lambda})=\langle\psi, \exp (i \boldsymbol{\lambda} \cdot \hat{\mathbf{P}}) \psi\rangle$, implying the desired result. The same result can also be obtained using the well known asymptotic formula (see, e.g., 69]) for the solution of the free Schrödinger equation with initial condition $\psi=\psi(\mathbf{x})$

$$
\psi_{T}(\mathbf{x}) \sim\left(\frac{m}{i T}\right)^{\frac{3}{2}} e^{i \frac{m \mathbf{x}^{2}}{2 \hbar T}} \tilde{\psi}\left(\frac{m \mathbf{x}}{T}\right) \quad \text { for } \quad T \rightarrow \infty .
$$
}


Then, for initial state $\Psi=\psi \otimes \Phi_{0}$ with suitable $\Phi_{0}$, where $\psi=\alpha \psi^{(+)}+\beta \psi^{(-)}$, the distribution of the random variable

$$
\Sigma_{z T}=F_{T}\left(\mathbf{X}_{T}\right)=\frac{2 m Z_{T}}{a T^{2}}
$$

converges as $T \rightarrow \infty$ to the spectral measure of $\sigma_{z}$, with values +1 and -1 occurring with probabilities $|\alpha|^{2}$ and $|\beta|^{2}$, respectively. ${ }^{29}$ This is so, just as with the momentum, because as $T \rightarrow \infty$ the operator $\frac{2 m \hat{Z}_{T}}{a T^{2}}$ converges to $\sigma_{z}$.

We remark that we've made use above of the fact that simple algebraic manipulations on the level of random variables correspond automatically to the same manipulations for the associated operators. More precisely, suppose that

$$
Z \mapsto A
$$

in the sense (of (2.37) ) that the distribution of the random variable $Z$ is given by the spectral measure for the self-adjoint operator $A$. Then it follows from (3.17) that

$$
f(Z) \rightarrow f(A)
$$

for any (Borel) function $f$. For example, since $\mathbf{X}_{T} \mapsto \hat{\mathbf{X}}_{T}, m \mathbf{X}_{T} / T \mapsto m \hat{\mathbf{X}}_{T} / T$, and since $Z_{T} \rightarrow$ $\hat{Z}_{T}, \frac{2 m Z_{T}}{a T^{2}} \rightarrow \frac{2 m \hat{Z}_{T}}{a T^{2}}$. Similarly, if a random variable $P \mapsto \hat{P}$, then $P^{2} /(2 m) \mapsto H_{0}=\hat{P}^{2} /(2 m)$. This is rather trivial, but it is not as trivial as the failure even to distinguish $Z$ and $\hat{Z}$ would make it seem.

\subsection{From Positive-Operator-Valued Measures to Experiments}

We wish here to point out that to a very considerable extent the association $\mathscr{E} \mapsto O(d \lambda)$ of experiments with POVMs is onto. It is more or less the case that every POVM arises from an experiment.

We have in mind two distinct remarks. First of all, it was pointed out in the first paragraph of Section 4.3 that every discrete POVM $O_{\alpha}$ (weak formal experiment) arises from some discrete experiment $\mathscr{E}$. Thus, for every POVM $O(d \lambda)$ there is a sequence $\mathscr{E}^{(n)}$ of discrete experiments for which the corresponding POVMs $O^{(n)}$ converge to $O$.

The second point we wish to make is that to the extent that every PVM arises from an experiment $\mathscr{E}=\left\{\Phi_{0}, U, F\right\}$, so too does every POVM. This is based on the fact, mentioned at the end of the introduction to Section 5, that every POVM $O(d \lambda)$ can be regarded as arising from the projection of a PVM $E(d \lambda)$, acting on $\mathcal{H}^{(1)}$, onto the subspace $\mathcal{H} \subset \mathcal{H}^{(1)}$. We may assume without loss of generality that both $\mathcal{H}$ and $\mathcal{H}^{(1)} \ominus \mathcal{H}$ are infinite dimensional (by some otherwise irrelevant enlargements if necessary). Thus we can identify $\mathcal{H}^{(1)}$ with $\mathcal{H} \otimes \mathcal{H}_{\text {apparatus }}{ }^{(1)}$ and the subspace with $\mathcal{H} \otimes \Phi_{0}^{(1)}$, for any choice of $\Phi_{0}^{(1)}$. Suppose now that there is an experiment $\mathscr{E}^{(1)}=\left\{\Phi_{0}^{(2)}, U, F\right\}$ that measures the PVM $E$ (i.e., that measures the observable $A=\int \lambda E(d \lambda)$ ) where $\Phi_{0}^{(2)} \in \mathcal{H}_{\text {apparatus }}{ }^{(2)}, U$ acts on $\mathcal{H} \otimes \mathcal{H}_{\text {apparatus }}$ where $\mathcal{H}_{\text {apparatus }}=\mathcal{H}_{\text {apparatus }}{ }^{(1)} \otimes \mathcal{H}_{\text {apparatus }}{ }^{(2)}$ and $F$ is a function of the configuration of the composite of the 3 systems: system, apparatus ${ }^{(1)}$ and apparatus ${ }^{(2)}$. Then, with $\Phi_{0}=\Phi_{0}^{(1)} \otimes \Phi_{0}^{(2)}, \mathscr{E}=\left\{\Phi_{0}, U, F\right\}$ is associated with the POVM $O$.

\footnotetext{
${ }^{29}$ For the Hamiltonian (5.7) no assumption on the initial state $\Psi$ is required here; however (5.7) will be a reasonably good approximation only when $\Psi$ has a suitable form, expressing in particular that the particle is appropriately moving towards the magnet.
} 


\subsection{Invariance Under Trivial Extension}

Suppose we change an experiment $\mathscr{E}$ to $\mathscr{E}^{\prime}$ by regarding its $x$-system as containing more of the universe that the $x$-system for $\mathscr{E}$, without in any way altering what is physically done in the experiment and how the result is specified. One would imagine that $\mathscr{E}^{\prime}$ would be equivalent to $\mathscr{E}$. This would, in fact, be trivially the case classically, as it would if $\mathscr{E}$ were a genuine measurement, in which case $\mathscr{E}^{\prime}$ would obviously measure the same thing as $\mathscr{E}$. This remains true for the more formal notion of measurement under consideration here. The only source of nontriviality in arriving at this conclusion is the fact that with $\mathscr{E}^{\prime}$ we have to deal with a different, larger class of initial wave functions.

We will say that $\mathscr{E}^{\prime}$ is a trivial extension of $\mathscr{E}$ if the only relevant difference between $\mathscr{E}$ and $\mathscr{E}^{\prime}$ is that the $x$-system for $\mathscr{E}^{\prime}$ has generic configuration $x^{\prime}=(x, \hat{x})$, whereas the $x$-system for $\mathscr{E}$ has generic configuration $x$. In particular, the unitary operator $U^{\prime}$ associated with $\mathscr{E} \prime$ has the form $U^{\prime}=U \otimes \hat{U}$, where $U$ is the unitary associated with $\mathscr{E}$, implementing the interaction of the $x$-system and the apparatus, while $\hat{U}$ is a unitary operator describing the independent evolution of the $\hat{x}$-system, and the calibration $F$ for $\mathscr{E}^{\prime}$ is the same as for $\mathscr{E}$. (Thus $F$ does not depend upon $\hat{x}$.)

The association of experiments with (generalized) observables (POVMs) is invariant under trivial extension: if $\mathscr{E} \mapsto O$ in the sense of (4.10) and $\mathscr{E}^{\prime}$ is a trivial extension of $\mathscr{E}$, then $\mathscr{E}^{\prime} \mapsto O \otimes I$, where $I$ is the identity on the Hilbert space of the $\hat{x}$-system.

To see this note that if $\mathscr{E} \mapsto O$ then the sesquilinear map $B$ arising from (5.2) for $\mathscr{E}$ ' is of the form

$$
B\left(\psi_{1} \otimes \hat{\psi}_{1}, \psi_{2} \otimes \hat{\psi}_{2}\right)=\left\langle\psi_{1}, O \psi_{2}\right\rangle\left\langle\hat{\psi}_{1}, \hat{\psi}_{2}\right\rangle
$$

on product wave functions $\psi^{\prime}=\psi \otimes \hat{\psi}$, which easily follows from the form of $U^{\prime}$ and the fact that $F$ doesn't depend upon $\hat{x}$, so that the $\hat{x}$-degrees of freedom can be integrated out. Thus the POVM $O^{\prime}$ for $\mathscr{E}^{\prime}$ agrees with $O \otimes I$ on product wave functions, and since such wave functions span the Hilbert space for the $(x, \hat{x})$-system, we have that $O^{\prime}=O \otimes I$. Thus $\mathscr{E}^{\prime} \mapsto O \otimes I$.

In other words, if $\mathscr{E}$ is a measurement of $O$, then $\mathscr{E}^{\prime}$ is a measurement of $O \otimes I$. In particular, if $\mathscr{E}$ is a measurement the self-adjoint operator $A$, then $\mathscr{E}^{\prime}$ is a measurement of $A \otimes I$. This result is not quite so trivial as it would be were it concerned with genuine measurements, rather than with the more formal notion under consideration here.

Now suppose that $\mathscr{E}^{\prime}$ is a trivial extension of a discrete experiment $\mathscr{E}$, with state transformations given by $R_{\alpha}$. Then the state transformations for $\mathscr{E}^{\prime}$ are given by $R_{\alpha}^{\prime}=R_{\alpha} \otimes \hat{U}$. This is so because $R_{\alpha}^{\prime}$ must agree with $R_{\alpha} \otimes \hat{U}$ on product wave functions $\psi^{\prime}=\psi \otimes \hat{\psi}$, and these span the Hilbert space of the $(x, \hat{x})$-system.

\subsection{POVMs and the Positions of Photons and Dirac Electrons}

We have indicated how POVMs emerge naturally in association with Bohmian experiments. We wish here to indicate a somewhat different role for a POVM: to describe the probability distribution of the actual (as opposed to measured ${ }^{30}$ ) position. The probability distribution of the position of a Dirac electron in the state $\psi$ is $\psi^{+} \psi$. This is given by a PVM $E(d \mathbf{x})$ on the one-particle Hilbert space $\mathcal{H}$ spanned by positive and negative energy electron wave functions. However the physical one-particle Hilbert-space $\mathcal{H}_{+}$consists solely of positive energy states, and

\footnotetext{
${ }^{30}$ The accurate measurement of the position of a Dirac electron is presumably impossible.
} 
this is not invariant under the projections $E$. Nonetheless the probability distribution of the position of the electron is given by the POVM $P_{+} E(d \mathbf{x}) P_{+}$acting on $\mathcal{H}_{+}$, where $P_{+}$is the orthogonal projection onto $\mathcal{H}_{+}$. Similarly, constraints on the photon wave function require the use of POVMs for the localization of photons [54, 3. ${ }^{31}$

\section{Density Matrices}

The notion of a density matrix, a positive (trace class) operator with unit trace on the Hilbert space of a system, is often regarded as providing the most general characterization of a quantum state of that system. According to the quantum formalism, when a system is described by the density matrix $W$, the expected value of an observable $A$ is given by $\operatorname{tr}(W A)$. If $A$ has $\mathrm{PVM} O$, and more generally for any POVM $O$, the probability that the (generalized) observable $O$ has value in $\Delta$ is given by

$$
\operatorname{Prob}(O \in \Delta)=\operatorname{tr}(W O(\Delta)) .
$$

A density matrix that is a one-dimensional projection, i.e., of the form $|\psi\rangle\langle\psi|$ where $\psi$ is a unit vector in the Hilbert space of the system, describes a pure state (namely, $\psi$ ), and a general density matrix can be decomposed into a mixture of pure states $\psi_{k}$,

$$
W=\sum_{k} p_{k}\left|\psi_{k}\right\rangle\left\langle\psi_{k}\right| \quad \text { where } \quad \sum_{k} p_{k}=1
$$

Naively, one might regard $p_{k}$ as the probability that the system is in the state $\psi_{k}$. This interpretation is, however, untenable, for a variety of reasons. First of all, the decomposition (6.2) is not unique. A density matrix $W$ that does not describe a pure state can be decomposed into pure states in a variety of different ways.

It is always possible to decompose a density matrix $W$ in such a way that its components $\psi_{k}$ are orthonormal. Such a decomposition will be unique except when $W$ is degenerate, i.e., when some $p_{k}$ 's coincide. For example, if $p_{1}=p_{2}$ we may replace $\psi_{1}$ and $\psi_{2}$ by any other orthonormal pair of vectors in the subspace spanned by $\psi_{1}$ and $\psi_{2}$. And even if $W$ were nondegenerate, it need not be the case that the system is in one of the states $\psi_{k}$ with probability $p_{k}$, because for any decomposition (6.2), regardless of whether the $\psi_{k}$ are orthogonal, if the wave function of the system were $\psi_{k}$ with probability $p_{k}$, this situation would be described by the density matrix $W$.

Thus a general density matrix carries no information - not even statistical information — about the actual wave function of the system. Moreover, a density matrix can describe a system that has no wave function at all! This happens when the system is a subsystem of a larger system whose wave function is entangled, i.e., does not properly factorize (in this case one usually speaks of the reduced density matrix of the subsystem).

This impossibility of interpreting density matrices as real mixtures of pure states has been regarded by many authors (e.g., von Neumann [74] and Landau [56]) as a further indication that quantum randomness is inexplicable within the realm of classical logic and probability. However, from the point of view of Bohmian mechanics, there is nothing mysterious about density matrices. Indeed, their role and status within the quantum formalism can be understood very easily in terms

\footnotetext{
${ }^{31}$ For example, on the one-photon level, both the proposal $\mathbf{\Psi}=\mathbf{E}+i \mathbf{B}$ (where $\mathbf{E}$ and $\mathbf{B}$ are the electric and the magnetic free fields) [12, and the proposal $\mathbf{\Psi}=\mathbf{A}$ (where $\mathbf{A}$ is the vector potential in the Coulomb gauge) [3], require the constraint $\boldsymbol{\nabla} \cdot \boldsymbol{\Psi}=0$.
} 
of the general framework of experiments of Section 5. (It can, we believe, be reasonably argued that even from the perspective of orthodox quantum theory, density matrices can be understood in a straightforward way.)

\subsection{Density Matrices and Bohmian Experiments}

Consider a general experiment $\mathscr{E} \mapsto O$ (see equation (4.10)) and suppose that the initial wave function of the system is random with probability distribution $p(d \psi$ ) (on the set of unit vectors in $\mathcal{H}$ ). Then nothing will change in the general argument of Section 5 except that now $\rho_{\psi}^{Z}$ in (4.10) and (5.2) should be interpreted as the conditional probability given $\psi$. It follows then from (6.1), using the fact that $\langle\psi, O(\Delta) \psi\rangle=\operatorname{tr}(|\psi\rangle\langle\psi| O(\Delta))$, that the probability that the result of $\mathscr{E}$ lies in $\Delta$ is given by

$$
\int p(d \psi)\langle\psi, O(\Delta) \psi\rangle=\operatorname{tr}\left(\int p(d \psi)|\psi\rangle\langle\psi| O(\Delta)\right)=\operatorname{tr}(W O(\Delta))
$$

where $^{32}$

$$
W \equiv \int p(d \psi)|\psi\rangle\langle\psi|
$$

is the ensemble density matrix arising from a random wave function with (ensemble) distribution $p$.

Now suppose that instead of having a random wave function, our system has no wave function at all because it is entangled with another system. Then there is still an object that can naturally be regarded as the state of our system, an object associated with the system itself in terms of which the results of experiments performed on our system can be simply expressed. This object is a density matrix $W$ and the results are governed by (6.1). $W$ is the reduced density matrix arising from the state of the larger system. This is more or less an immediate consequence of invariance under trivial extension, described in Section 5.7

Consider a trivial extension $\mathscr{E}^{\prime}$ of an experiment $\mathscr{E} \mapsto O$ on our system-precisely what we must consider if the larger system has a wave function $\psi^{\prime}$ while our (smaller) system does not. The probability that the result of $\mathscr{E}^{\prime}$ lies in $\Delta$ is given by

$$
\left\langle\psi^{\prime}, O(\Delta) \otimes I \psi^{\prime}\right\rangle=\operatorname{tr}^{\prime}\left(\left|\psi^{\prime}\right\rangle\left\langle\psi^{\prime}\right| O(\Delta) \otimes I\right)=\operatorname{tr}(W O(\Delta))
$$

where $\operatorname{tr}^{\prime}$ is the trace for the $x^{\prime}$-system (the big system) and $\operatorname{tr}$ is the trace for the $x$-system. In agreement with standard quantum mechanics, the last equality of (6.5) defines $W$ as the reduced density matrix of the $x$-system, i.e,

$$
W \equiv \widehat{\operatorname{tr}}\left(\left|\psi^{\prime}\right\rangle\left\langle\psi^{\prime}\right|\right)
$$

where $\widehat{t r}$ denotes the partial trace over the coordinates of the $\hat{x}$-system.

\footnotetext{
${ }^{32}$ Note that since $p$ is a probability measure on the unit sphere in $\mathcal{H}, W$ is a positive trace class operator with unit trace.
} 


\subsection{Strong Experiments and Density Matrices}

A strong formal experiment $\mathcal{E} \equiv\left\{\lambda_{\alpha}, R_{\alpha}\right\}$ generates state transformations $\psi \rightarrow R_{\alpha} \psi$. This suggests the following action on an initial state described by a density matrix $W$ : When the outcome is $\alpha$, we have the transformation

$$
W \rightarrow \frac{\mathcal{R}_{\alpha} W}{\operatorname{tr}\left(\mathcal{R}_{\alpha} W\right)} \equiv \frac{R_{\alpha} W R_{\alpha}^{*}}{\operatorname{tr}\left(R_{\alpha} W R_{\alpha}^{*}\right)}
$$

where

$$
\mathcal{R}_{\alpha} W=R_{\alpha} W R_{\alpha}^{*} .
$$

After all, (6.7) is a density matrix naturally associated with $R_{\alpha}$ and $W$, and it agrees with $\psi \rightarrow R_{\alpha} \psi$ for a pure state, $W=|\psi\rangle\langle\psi|$. In order to show that (6.7) is indeed correct, we must verify it for the two different ways in which our system might be assigned a density matrix $W$, i.e., for $W$ an ensemble density matrix and for $W$ a reduced density matrix.

Suppose the initial wave function is random, with distribution $p(d \psi)$. Then the initial state of our system is given by the density matrix (6.4). When the outcome $\alpha$ is obtained, two changes must be made in (6.4) to reflect this information: $|\psi\rangle\langle\psi|$ must be replaced by $\left(R_{\alpha}|\psi\rangle\langle\psi| R_{\alpha}^{*}\right) /\left\|R_{\alpha} \psi\right\|^{2}$, and $p(d \psi)$ must be replaced by $p(d \psi \mid \alpha)$, the conditional distribution of the initial wave function given that the outcome is $\alpha$. For the latter we have

$$
p(d \psi \mid \alpha)=\frac{\left\|R_{\alpha} \psi\right\|^{2}}{\operatorname{tr}\left(R_{\alpha} W R_{\alpha}^{*}\right)} p(d \psi)
$$

$\left(\left\|R_{\alpha} \psi\right\|^{2} p(d \psi)\right.$ is the joint distribution of $\psi$ and $\alpha$ and the denominator is the probability of obtaining the outcome $\alpha$.) Therefore $W$ undergoes the transformation

$$
W=\int p(d \psi)|\psi\rangle\langle\psi| \rightarrow \int p(d \psi \mid \alpha) \frac{R_{\alpha}|\psi\rangle\langle\psi| R_{\alpha}^{*}}{\left\|R_{\alpha} \psi\right\|^{2}}=\int p(d \psi) \frac{R_{\alpha}|\psi\rangle\langle\psi| R_{\alpha}^{*}}{\operatorname{tr}\left(R_{\alpha} W R_{\alpha}^{*}\right)}=\frac{R_{\alpha} W R_{\alpha}^{*}}{\operatorname{tr}\left(R_{\alpha} W R_{\alpha}^{*}\right)}
$$

We wish to emphasize that this demonstrates in particular the nontrivial fact that the density matrix $\mathcal{R}_{\alpha} W / \operatorname{tr}\left(\mathcal{R}_{\alpha} W\right)$ produced by the experiment depends only upon the initial density matrix $W$. Though $W$ can arise in many different ways, corresponding to the multiplicity of different probability distributions $p(d \psi)$ yielding $W$ via (6.4), insofar as the final state is concerned, these differences don't matter.

This does not, however, establish (6.7) when $W$ arises not from a random wave function but as a reduced density matrix. To deal with this case we consider a trivial extension $\mathscr{E} \prime$ of a discrete experiment $\mathscr{E}$ with state transformations $R_{\alpha}$. Then $\mathscr{E}^{\prime}$ has state transformations $R_{\alpha} \otimes \hat{U}$ (see Section [5.7). Thus, when the initial state of the $x^{\prime}$-system is $\psi^{\prime}$, the final state of the $x$-system is given by the partial trace

$$
\begin{gathered}
\frac{\widehat{\operatorname{tr}}\left(R_{\alpha} \otimes \hat{U}\left|\psi^{\prime}\right\rangle\left\langle\psi^{\prime}\right| R_{\alpha}^{*} \otimes \hat{U}^{*}\right)}{\operatorname{tr}^{\prime}\left(R_{\alpha} \otimes \hat{U}\left|\psi^{\prime}\right\rangle\left\langle\psi^{\prime}\right| R_{\alpha}^{*} \otimes \hat{U}^{*}\right)}=\frac{\widehat{\operatorname{tr}}\left(R_{\alpha} \otimes I\left|\psi^{\prime}\right\rangle\left\langle\psi^{\prime}\right| R_{\alpha}^{*} \otimes I\right)}{\operatorname{tr}^{\prime}\left(R_{\alpha} \otimes I\left|\psi^{\prime}\right\rangle\left\langle\psi^{\prime}\right| R_{\alpha}^{*} \otimes I\right)}=\frac{R_{\alpha} \widehat{\operatorname{tr}}\left(\left|\psi^{\prime}\right\rangle\left\langle\psi^{\prime}\right|\right) R_{\alpha}^{*}}{\operatorname{tr}\left(R_{\alpha} \widehat{\operatorname{tr}}\left(\left|\psi^{\prime}\right\rangle\left\langle\psi^{\prime}\right|\right) R_{\alpha}^{*}\right)} \\
=\frac{R_{\alpha} W R_{\alpha}^{*}}{\operatorname{tr}\left(R_{\alpha} W R_{\alpha}^{*}\right)}
\end{gathered}
$$

where the cyclicity of the trace has been used. 
To sum up, when a strong experiment $\mathcal{E} \equiv\left\{\lambda_{\alpha}, R_{\alpha}\right\}$ is performed on a system described by the initial density matrix $W$ and the outcome $\alpha$ is obtained, the final density matrix is given by (6.7); moreover, from the results of the previous section it follows that the outcome $\alpha$ will occur with probability

$$
p_{\alpha}=\operatorname{tr}\left(W O_{\alpha}\right)=\operatorname{tr}\left(W R_{\alpha}^{*} R_{\alpha}\right)=\operatorname{tr}\left(\mathcal{R}_{\alpha} W\right),
$$

where the last equality follows from the cyclicity of the trace.

\subsection{The Notion of Instrument}

We shall briefly comment on the relationship between the notion of strong formal experiment and that of instrument (or effect) discussed by Davies [21].

Consider an experiment $\mathcal{E} \equiv\left\{\lambda_{\alpha}, R_{\alpha}\right\}$ on a system with initial density matrix $W$. Then a natural object associated with $\mathcal{E}$ is the set function

$$
\mathcal{R}(\Delta) W \equiv \sum_{\lambda_{\alpha} \in \Delta} \mathcal{R}_{\alpha} W=\sum_{\lambda_{\alpha} \in \Delta} R_{\alpha} W R_{\alpha}^{*}
$$

The set function $\mathcal{R}: \Delta \mapsto \mathcal{R}(\Delta)$ compactly expresses both the statistics of $\mathcal{E}$ for a general initial system density matrix $W$ and the effect of $\mathcal{E}$ on $W$ conditioned on the occurrence of the event "the result of $\mathcal{E}$ is in $\Delta$ ".

To see this, note first that it follows from (6.9) that the probability that the result of the experiment lies in the set $\Delta$ is given by

$$
p(\Delta)=\operatorname{tr}(\mathcal{R}(\Delta) W)
$$

The conditional distribution $p(\alpha \mid \Delta)$ that the outcome is $\alpha$ given that the result $\lambda_{\alpha} \in \Delta$ is then $\operatorname{tr}\left(\mathcal{R}_{\alpha} W\right) / \operatorname{tr}(\mathcal{R}(\Delta) W)$. The density matrix that reflects the knowledge that the result is in $\alpha$, obtained by averaging (6.7) over $\Delta$ using $p(\alpha \mid \Delta)$, is thus $\mathcal{R}(\Delta) W / \operatorname{tr}(\mathcal{R}(\Delta) W)$.

It follows from (6.10) that $\mathcal{R}$ is a countably additive set function whose values are positive preserving linear transformations in the space of trace-class operators in $\mathcal{H}$. Any map with these properties, not necessarily of the special form (6.10), is called an instrument.

\subsection{On the State Description Provided by Density Matrices}

So far we have followed the standard terminology and have spoken of a density matrix as describing the state of a physical system. It is important to appreciate, however, that this is merely a frequently convenient way of speaking, for Bohmian mechanics as well as for orthodox quantum theory. Insofar as Bohmian mechanics is concerned, the significance of density matrices is neither more nor less than what is implied by their role in the quantum formalism as described in Sections 6.1 and 6.2. While many aspects of the notion of (effective) wave function extend to density matrices, in particular with respect to weak and strong experiments, density matrices lack the dynamical implications of wave functions for the evolution of the configuration, a point that has been emphasized by Bell [7]:

In the de Broglie-Bohm theory a fundamental significance is given to the wave function, and it cannot be transferred to the density matrix. ... Of course the density matrix retains all its usual practical utility in connection with quantum statistics. 
That this is so should be reasonably clear, since it is the wave function that determines, in Bohmian mechanics, the evolution of the configuration, and the density matrix of a system does not determine its wave function, even statistically. To underline the point we shall recall the analysis of Bell [7: Consider a particle described by a density matrix $W_{t}$ evolving autonomously, so that $W_{t}=U_{t} W_{0} U_{t}^{-1}$, where $U_{t}$ is the unitary group generated by a Schrödinger Hamiltonian. Then $\rho^{W_{t}}(x) \equiv W_{t}(x, x) \equiv\left\langle x\left|W_{t}\right| x\right\rangle$ gives the probability distribution of the position of the particle. Note that $\rho^{W}$ satisfies the continuity equation

$$
\frac{\partial \rho^{W}}{\partial t}+\operatorname{div} J^{W}=0 \quad \text { where } \quad J^{W}(x)=\frac{\hbar}{m} \operatorname{Im}\left[\nabla_{x} W\left(x, x^{\prime}\right)\right]_{x^{\prime}=x} .
$$

This might suggest that the velocity of the particle should be given by $v=J^{W} / \rho^{W}$, which indeed agrees with the usual formula when $W$ is a pure state $\left(W\left(x, x^{\prime}\right)=\psi(x) \psi^{*}\left(x^{\prime}\right)\right)$. However, this extension of the usual formula to arbitrary density matrices, though mathematically "natural," is not consistent with what Bohmian mechanics prescribes for the evolution of the configuration. Consider, for example, the situation in which the wave function of a particle is random, either $\psi_{1}$ or $\psi_{2}$, with equal probability. Then the density matrix is $W\left(x, x^{\prime}\right)=\frac{1}{2}\left(\psi_{1}(x) \psi_{1}^{*}\left(x^{\prime}\right)+\psi_{2}(x) \psi_{2}^{*}\left(x^{\prime}\right)\right)$. But the velocity of the particle will be always $e i$ ther $v_{1}$ or $v_{2}$ (according to whether the actual wave function is $\psi_{1}$ or $\psi_{2}$ ), and-unless $\psi_{1}$ and $\psi_{2}$ have disjoint supports - this does not agree with $J^{W} / \rho^{W}$, an average of $v_{1}$ and $v_{2}$.

What we have just said is correct, however, only when spin is ignored. For particles with spin a novel kind of density matrix emerges, a conditional density matrix, analogous to the conditional wave function (2.6) and with an analogous dynamical role: Even though no conditional wave function need exist for a system entangled with its environment when spin is taken into account, a conditional density matrix $W$ always exists, and is such that the velocity of the system is indeed given by $J^{W} / \rho^{W}$. See 31 for details.

A final remark: the statistical role of density matrices is basically different from that provided by statistical ensembles, e.g, by Gibbs states in classical statistical mechanics. This is because, as mentioned earlier, even when it describes a random wave function via (6.4), a density matrix $W$ does not determine the ensemble $p(d \psi)$ from which it emerges. The map defined by (6.4) from probability measures $p$ on the unit sphere in $\mathcal{H}$ to density matrices $W$ is many-to-one. ${ }^{33}$ Consider, for example, the density matrix $\frac{1}{n} I$ where $I$ is the identity operator on an $n$-dimensional Hilbert space $\mathcal{H}$. Then a uniform distribution over the vectors of any given orthonormal basis of $\mathcal{H}$ leads to this density matrix, as well as does the continuous uniform measure on the sphere $\|\psi\|=1$. However, since the statistical distribution of the results of any experiment depends on $p$ only through $W$, different $p$ 's associated with the same $W$ are empirically equivalent in the sense that they can't be distinguished by experiments performed on a system prepared somehow in the state $W$.

\footnotetext{
${ }^{33}$ This is relevant to the foundations of quantum statistical mechanics, for which the state of an isolated thermodynamic system is usually described by the microcanonical density matrix $\mathcal{Z}^{-1} \delta(H-E)$, where $\mathcal{Z}=$ $\operatorname{tr} \delta(H-E)$ is the partition function. Which ensemble of wave functions should be regarded as forming the thermodynamic ensemble? A natural choice is the uniform measure on the subspace $H=E$, which should be thought of as fattened in the usual way. Note that this choice is quite distinct from another one that people often have in mind: a uniform distribution over a basis of energy eigenstates of the appropriate energy. Depending upon the choice made, we obtain different notions of typical equilibrium wave function.
} 


\section{Genuine Measurements}

We have so far discussed various interactions between a system and an apparatus relevant to the quantum measurement formalism, from the very special ones formalized by "ideal measurements" to the general situation described in section 5. It is important to recognize that nowhere in this discussion was there any implication that anything was actually being measured. The fact that an interaction with an apparatus leads to a pointer orientation that we call the result of the experiment or "measurement" in no way implies that this result reflects anything of significance concerning the system under investigation, let alone that it reveals some preexisting property of the system - and this is what is supposed to be meant by the word measurement. After all [72], "any old playing around with an indicating instrument in the vicinity of another body, whereby at any old time one then takes a reading, can hardly be called a measurement of this body," and the fact the experiment happens to be associated, say, with a self-adjoint operator in the manner we have described, so that the experiment is spoken of, in the quantum formalism, as a measurement of the corresponding observable, certainly offers little support for using language in this way.

We shall elaborate on this point later on. For now we wish to observe that the very generality of our analysis, particularly that of section 5, covering as it does all possible interactions between system and apparatus, covers as well those particular situations that in fact are genuine measurements. This allows us to make some definite statements about what can be measured in Bohmian mechanics.

For a physical quantity, describing an objective property of a system, to be measurable means that it is possible to perform an experiment on the system that measures the quantity, i.e., an experiment whose result conveys its value. In Bohmian mechanics a physical quantity $\xi$ is expressed by a function

$$
\xi=f(X, \psi)
$$

of the complete state $(X, \psi)$ of the system. An experiment $\mathscr{E}$ measuring $\xi$ is thus one whose result $Z=F\left(X_{T}, Y_{T}\right) \equiv Z(X, Y, \Psi)$ equals $\xi=f(X, \psi) \equiv \xi(X, \psi)$,

$$
Z(X, Y, \Psi)=\xi(X, \psi)
$$

where $X, Y, \psi$ and $\Psi$ refer, as in Section 5, to the initial state of system and apparatus, immediately prior to the measurement, and where the equality should be regarded as approximate, holding to any desired degree of accuracy.

The most basic quantities are, of course, the state components themselves, namely $X$ and $\psi$, as well as the velocities

$$
\mathbf{v}_{k}=\frac{\hbar}{m_{k}} \operatorname{Im} \frac{\nabla_{k} \psi(X)}{\psi(X)}
$$

of the particles. One might also consider quantities describing the future behavior of the system, such as the configuration of an isolated system at a later time, or the time of escape of a particle from a specified region, or the asymptotic velocity discussed in Section 5.5. (Because the dynamics is deterministic, all of these quantities are functions of the initial state of the system and are thus of the form (7.1).)

We wish to make a few remarks about the measurability of these quantities. In particular, we wish to mention, as an immediate consequence of the analysis at the beginning of Section 5, a condition that must be satisfied by any quantity if it is to be measurable. 


\subsection{A Necessary Condition for Measurability}

Consider any experiment $\mathscr{E}$ measuring a physical quantity $\xi$. We showed in Section 5 that the statistics of the result $Z$ of $\mathscr{E}$ must be governed by a POVM, i.e., that the probability distribution of $Z$ must be given by a measure-valued quadratic map on the system Hilbert space $\mathcal{H}$. Thus, by (7.2),

$\xi$ is measurable only if its probability distribution $\mu_{\xi}^{\psi}$ is a measure-valued quadratic map on $\mathcal{H}$.

As indicated earlier, the position $\mathbf{X}$ and the asymptotic velocity or momentum $\mathbf{P}$ have distributions quadratic in $\psi$, namely $\mu_{\mathbf{X}}^{\psi}(d \mathbf{x})=|\psi(\mathbf{x})|^{2}$ and $\mu_{\mathbf{P}}^{\psi}(d \mathbf{p})=|\tilde{\psi}(\mathbf{p})|^{2}$, respectively. Moreover, they are both measurable, basically because suitable local interactions exist to establish appropriate correlations with the relevant macroscopic variables. For example, in a bubble chamber a particle following a definite path triggers a chain of reactions that leads to the formation of (macroscopic) bubbles along the path.

The point we wish to make now, however, is simply this: the measurability of these quantities is not a consequence of the fact that these quantities obey this measurability condition. We emphasize that this condition is merely a necessary condition for measurability, and not a sufficient one. While it does follow that if $\xi$ satisfies this condition there exists a discrete experiment that is an approximate formal measurement of $\xi$ (in the sense that the distribution of the result of the experiment is approximately $\mu_{\xi}^{\psi}$ ), this experiment need not provide a genuine measurement of $\xi$ because the interactions required for its implementation need not exist and because, even if they did, the result $Z$ of the experiment might not be related to the quantity $\xi$ in the right way, i.e, via (7.2).

We now wish to illustrate the use of this condition, first transforming it into a weaker but more convenient form. Note that any quadratic map $\mu^{\psi}$ must satisfy

$$
\mu^{\psi_{1}+\psi_{2}}+\mu^{\psi_{1}-\psi_{2}}=2\left(\mu^{\psi_{1}}+\mu^{\psi_{2}}\right)
$$

and thus if $\mu^{\psi}$ is also positive we have the inequality

$$
\mu^{\psi_{1}+\psi_{2}} \leq 2\left(\mu^{\psi_{1}}+\mu^{\psi_{2}}\right)
$$

Thus it follows from (7.4) that a quantity ${ }^{34}$

$\xi$ must fail to be measurable if it has a possible value (one with nonvanishing probability or probability density) when the wave function of the system is $\psi_{1}+\psi_{2}$ that is neither a possible value when the wave function is $\psi_{1}$ nor a possible value when the wave function is $\psi_{2}$.

(Here neither $\psi_{1}$ nor $\psi_{2}$ need be normalized.)

\footnotetext{
${ }^{34}$ This conclusion is also a more or less direct consequence of the linearity of the Schrödinger evolution: If $\psi_{i} \otimes \Phi_{0} \mapsto \Psi_{i}$ for all $i$, then $\sum \psi_{i} \otimes \Phi_{0} \mapsto \sum \Psi_{i}$. But, again, our purpose here has been mainly to illustrate the use of the measurability condition itself.
} 


\subsection{The Nonmeasurability of Velocity, Wave Function and Determin- istic Quantities}

It is an immediate consequence of (17.6) that neither the velocity nor the wave function is measurable, the latter because the value " $\psi_{1}+\psi_{2}$ " is neither " $\psi_{1}$ " nor " $\psi_{2}$," and the former because every wave function $\psi$ may be written as $\psi=\psi_{1}+\psi_{2}$ where $\psi_{1}$ is the real part of $\psi$ and $\psi_{2}$ is $i$ times the imaginary part of $\psi$, for both of which the velocity (of whatever particle) is 0 .

Note that this is a very strong and, in a sense, surprising conclusion, in that it establishes the impossibility of measuring what is, after all, a most basic dynamical variable for a deterministic mechanical theory of particles in motion. It should probably be regarded as even more surprising that the proof that the velocity - or wave function - is not measurable seems to rely almost on nothing, in effect just on the linearity of the evolution of the wave function. However, one should not overlook the crucial role of quantum equilibrium.

We observe that the nonmeasurability of the wave function is related to the impossibility of copying the wave function. (This question arises sometimes in the form, "Can one clone the wave function?" [36, 78, 37.) Copying would be accomplished, for example, by an interaction leading, for all $\psi$, from $\psi \otimes \phi_{0} \otimes \Phi_{0}$ to $\psi \otimes \psi \otimes \Phi$, but this is clearly incompatible with unitarity. We wish here merely to remark that the impossibility of cloning can also be regarded as a consequence of the nonmeasurability of the wave function. In fact, were cloning possible one could - by making many copies - measure the wave function by performing suitable measurements on the various copies. After all, any wave function $\psi$ is determined by $\langle\psi, A \psi\rangle$ for sufficiently many observables $A$ and these expectation values can of course be computed using a sufficiently large ensemble.

By a deterministic quantity we mean any function $\xi=f(\psi)$ of the wave function alone (which thus does not inherit any irreducible randomness associated with the random configuration $X)$. It follows easily from (7.6) that no (nontrivial) deterministic quantity is measurable. ${ }^{35}$ In particular, the mean value $\langle\psi, A \psi\rangle$ of an observable $A$ (not a multiple of the identity) is not measurablethough it would be were it possible to copy the wave function, and it can of course be measured by a nonlinear experiment, see Section 7.4 .

\subsection{Initial Values and Final Values}

Measurement is a tricky business. In particular, one may wonder how, if it is not measurable, we are ever able to know the wave function of a system - which in orthodox quantum theory often seems to be the only thing that we do know about it.

In this regard, it is important to appreciate that we were concerned in the previous section only with initial values, with the wave function and the velocity prior to the measurement. We shall now briefly comment upon the measurability of final values, produced by the experiment.

The nonmeasurability argument of Section 7.2 does not cover final values. This may be appreciated by noting that the crucial ingredient in the analysis involves a fundamental timeasymmetry: The probability distribution $\mu^{\psi}$ of the result of an experiment is a quadratic functional of the initial wave function $\psi$, not the final one - of which it is not a functional at all. Moreover, the final velocity can indeed be measured, by a momentum measurement as described in Section 5.5. (That such a measurement yields also the final velocity follows from the formula in footnote 28 for the asymptotic wave function.) And the final wave function can be measured

\footnotetext{
${ }^{35}$ Note also that $\mu_{\xi}^{\psi}(d \lambda)=\delta(\lambda-f(\psi)) d \lambda$ seems manifestly nonquadratic in $\psi$ (unless $f$ is constant).
} 
by an ideal measurement of any nondegenerate observable, and more generally by any strong formal measurement whose subspaces $\mathcal{H}_{\alpha}$ are one-dimensional, see Section 3.5. If the outcome is $\alpha$, the final wave function is $R_{\alpha} \psi=R_{\alpha} P_{\mathcal{H}_{\alpha}} \psi$, which is independent of the initial wave function $\psi$ (up to a scalar multiple).

We also wish to remark that this distinction between measurements of initial values and measurements of final values has no genuine significance for passive measurements, that merely reveal preexisting properties without in any way affecting the measured system. However, quantum measurements are usually active; for example, an ideal measurement transforms the wave function of the system into an eigenstate of the measured observable. But passive or active, a measurement, by its very meaning, is concerned strictly speaking with properties of a system just before its performance, i.e., with initial values. At the same time, to the extent that any property of a system is conveyed by a typical quantum "measurement," it is a property defined by a final value.

For example, according to orthodox quantum theory a position measurement on a particle with a spread-out wave function, to the extent that it measures anything at all, measures the final position of the particle, created by the measurement, rather than the initial position, which is generally regarded as not existing prior to the measurement. And even in Bohmian mechanics, in which such a measurement may indeed reveal the initial position, which - if the measurement is suitably performed - will agree with the final position, this measurement will still be active since the wave function of the system must be transformed by the measurement into one that is compatible with the sharper knowledge of the position that it provides, see Section 2.1.

\subsection{Nonlinear Measurements and the Role of Prior Information}

The basic idea of measurement is predicated on initial ignorance. We think of a measurement of a property of a system as conveying that property by a procedure that does not seriously depend upon the state of the system, ${ }^{36}$ any details of which must after all be unknown prior to at least some engagement with the system. Be that as it may, the notion of measurement as codified by the quantum formalism is indeed rooted in a standpoint of ignorance: the experimental procedures involved in the measurement do not depend upon the state of the measured system. And our entire discussion of measurement up to now has been based upon that very assumption, that $\mathscr{E}$ itself does not depend on $\psi$ (and certainly not on $X$ ).

If, however, some prior information on the initial system wave function $\psi$ were available, we could exploit this information to measure quantities that would otherwise fail to be measurable. For example, for a single-particle system, if we somehow knew its initial wave function $\psi$ then a measurement of the initial position of the particle would convey its initial velocity as well, via (7.3) - even though, as we have shown, this quantity isn't measurable without such prior information.

By a nonlinear measurement or experiment $\mathscr{E}=\mathscr{E}^{\psi}$ we mean one in which, unlike those considered so far, one or more of the defining characteristics of the experiment depends upon $\psi$.

\footnotetext{
${ }^{36}$ This statement must be taken with a grain of salt. Some things must be known about the system prior to measurement, for example, that it is in the vicinity the measurement apparatus, or that an atom whose angular momentum we wish to measure is moving towards the relevant Stern Gerlach magnets, as well as a host of similar, often unnoticed, pieces of information. This sort of thing does not much matter for our purposes in this paper and can be safely ignored. Taking them into account would introduce pointless complications without affecting the analysis in an essential way.
} 
For example, in the measurement of the initial velocity described in the previous paragraph, the calibration function $F=F^{\psi}$ depends upon $\psi \cdot{ }^{37}$ More generally we might have that $U=U^{\psi}$ or $\Phi_{0}=\Phi_{0}^{\psi}$.

The wave function can of course be measured by a nonlinear measurement - just let $F^{\psi} \equiv \psi$. Somewhat less trivially, the initial wave function can be measured, at least formally, if it is known to be a member of a given orthonormal basis, by measuring any nondegenerate observable whose eigenvectors form that basis. The proposals of Aharonov, Anandan and Vaidman [1] for measuring the wave function, though very interesting, are of this character - they involve nonlinear measurements that depend upon a choice of basis containing $\psi$-and thus remain controversial. ${ }^{38}$

\subsection{A Position Measurement that Does not Measure Position}

We began this section by observing that what is spoken of as a measurement in quantum theory need not really measure anything. We mentioned, however, that in Bohmian mechanics the position can be measured, and the experiment that accomplishes this would of course be a measurement of the position operator. We wish here to point out, by means of a very simple example, that the converse is not true, i.e., that a measurement of the position operator need not be a measurement of the position.

Consider the harmonic oscillator in 2 dimensions with Hamiltonian

$$
H=-\frac{\hbar^{2}}{2 m}\left(\frac{\partial^{2}}{\partial x^{2}}+\frac{\partial^{2}}{\partial y^{2}}\right)+\frac{\omega^{2} m}{2}\left(x^{2}+y^{2}\right) \text {. }
$$

Except for an irrelevant time-dependent phase factor, the evolution $\psi_{t}$ is periodic, with period $\tau=2 \pi / \omega$. The Bohm motion of the particle, however, need not have period $\tau$. For example, the $(n=1, m=1)$-state, which in polar coordinates is of the form

$$
\psi_{t}(r, \phi)=\frac{m \omega}{\hbar \sqrt{\pi}} r e^{-\frac{m \omega}{2 \hbar} r^{2}} e^{i \phi} e^{-i \frac{3}{2} \omega t}
$$

generates a circular motion of the particle around the origin with angular velocity $\hbar /\left(m r^{2}\right)$, and hence with periodicity depending upon the initial position of the particle - the closer to the origin, the faster the rotation. Thus, in general,

$$
\mathbf{X}_{\tau} \neq \mathbf{X}_{0}
$$

Nonetheless, $\mathbf{X}_{\tau}$ and $\mathbf{X}_{0}$ are identically distributed random variables, since $\left|\psi_{\tau}\right|^{2}=\left|\psi_{0}\right|^{2} \equiv|\psi|^{2}$.

We may now focus on two different experiments: Let $\mathscr{E}$ be a measurement of the actual position $\mathbf{X}_{0}$, the initial position, and hence of the position operator, and let $\mathscr{E} \prime$ be an experiment beginning at the same time as $\mathscr{E}$ but in which it is the position $\mathbf{X}_{\tau}$ at time $\tau$ that is actually measured. Since for all $\psi$ the result of $\mathscr{E}^{\prime}$ has the same distribution as the result of $\mathscr{E}, \mathscr{E}$ is also a measurement of the position operator. But $\mathscr{E}^{\prime}$ is not a measurement of the initial position since

\footnotetext{
${ }^{37}$ Suppose that $Z_{1}=F_{1}\left(Q_{T}\right)=X$ is the result of the measurement of the initial position. Then $F^{\psi}=G^{\psi} \circ F_{1}$ where $G^{\psi}(\cdot)=\frac{\hbar}{m} \operatorname{Im} \frac{\nabla \psi}{\psi}(\cdot)$.

${ }^{38}$ In one of their proposals the wave function is "protected" by a procedure that depends upon the basis; in another, involving adiabatic interactions, $\psi$ must be a nondegenerate eigenstate of the Hamiltonian $H$ of the system, but it is not necessary that the latter be known.
} 
the position at time $\tau$ does not in general agree with the initial position: A measurement of the position at time $\tau$ is not a measurement of the position at time 0 . Thus, while a measurement of position is always a measurement of the position operator,

\section{A measurement of the position operator is not necessarily a genuine measurement of position!}

\subsection{Theory Dependence of Measurement}

The harmonic oscillator example provides a simple illustration of an elementary point that is often ignored: in discussions of measurement it is well to keep in mind the theory under consideration. The theory we have been considering here has been Bohmian mechanics. If, instead, we were to analyze the harmonic oscillator experiments described above using different theories our conclusions about results of measurements would in general be rather different, even if the different theories were empirically equivalent. So we shall analyze the above experiment $\mathscr{E}^{\prime}$ in terms of various other formulations or interpretations of quantum theory.

In strict orthodox quantum theory there is no such thing as a genuine particle, and thus there is no such thing as the genuine position of a particle. There is, however, a kind of operational definition of position, in the sense of an experimental setup, where a measurement device yields results the statistics of which are given by the position operator.

In naive orthodox quantum theory one does speak loosely about a particle and its position, which is thought of - in a somewhat uncritical way — as being revealed by measuring the position operator. Any experiment that yields statistics given by the position operator is considered a genuine measurement of the particle's position. ${ }^{39}$ Thus $\mathscr{E}^{\prime}$ would be considered as a measurement of the position of the particle at time zero.

The decoherent (or consistent) histories formulation of quantum mechanics [35, 65, 46] is concerned with the probabilities of certain coarse-grained histories, given by the specification of finite sequences of events, associated with projection operators, together with their times of occurrence. These probabilities are regarded as governing the occurrence of the histories, regardless of whether any of the events are measured or observed, but when they are observed, the probabilities of the observed histories are the same as those of the unobserved histories. The experiments $\mathscr{E}$ and $\mathscr{E}^{\prime}$ are measurements of single-event histories corresponding to the position of the particle at time 0 and at time $\tau$, respectively. Since the Heisenberg position operators $\hat{\mathbf{X}}_{\tau}=\hat{\mathbf{X}}_{0}$ for the harmonic oscillator, it happens to be the case, according to the decoherent histories formulation of quantum mechanics, that for this system the position of the particle at time $\tau$ is the same as its position at time 0 when the positions are unobserved, and that $\mathscr{E}^{\prime}$ in fact measures the position of the particle at time 0 (as well as the position at time $\tau$ ).

The spontaneous localization or dynamical reduction models [38, 40] are versions of quantum theory in which there are no genuine particles; in these theories reality is represented by the wave function alone (or, more accurately, by entities entirely determined by the wave function). In these models Schrödinger's equation is modified by the addition of a stochastic term that causes the wave function to collapse during measurement in a manner more or less consistent with the quantum formalism. In particular, the performance of $\mathscr{E}$ or $\mathscr{E}^{\prime}$ would lead to a random collapse of the oscillator wave function onto a narrow spatial region, which might be spoken of

\footnotetext{
${ }^{39}$ This, and the failure to appreciate the theory dependence of measurements, has been a source of unfounded criticisms of Bohmian mechanics(see [33, 24, 22]).
} 
as the position of the particle at the relevant time. But $\mathscr{E}^{\prime}$ could not be regarded in any sense as measuring the position at time 0 , because the localization does not occur for $\mathscr{E}^{\prime}$ until time $\tau$.

Finally we mention stochastic mechanics 64], a theory ontologically very similar to Bohmian mechanics in that the basic entities with which it is concerned are particles described by their positions. Unlike Bohmian mechanics, however, the positions evolve randomly, according to a diffusion process. Just as with Bohmian mechanics, for stochastic mechanics the experiment $\mathscr{E}^{\prime}$ is not a measurement of the position at time zero, but in contrast to the situation in Bohmian mechanics, where the result of the position measurement at time $\tau$ determines, given the wave function, the position at time zero (via the Bohmian equation of motion), this is not so in stochastic mechanics because of the randomness of the motion.

\section{Hidden Variables}

The issue of hidden variables concerns the question of whether quantum randomness arises in a completely ordinary manner, merely from the fact that in orthodox quantum theory we deal with an incomplete description of a quantum system. According to the hidden-variables hypothesis, if we had at our disposal a sufficiently complete description of the system, provided by supplementary parameters traditionally called hidden variables, the totality of which is usually denoted by $\lambda$, the behavior of the system would thereby be determined, as a function of $\lambda$ (and the wave function). In such a hidden-variables theory, the randomness in results of measurements would arise solely from randomness in the unknown variables $\lambda$. On the basis of a variety of "impossibility theorems," the hidden-variables hypothesis has been widely regarded as having been discredited.

Note that Bohmian mechanics is just such a hidden-variables theory, with the hidden variables $\lambda$ given by the configuration $Q$ of the total system. We have seen in particular that in a Bohmian experiment, the result $Z$ is determined by the initial configuration $Q=(X, Y)$ of the system and apparatus. Nonetheless, there remains much confusion about the relationship between Bohmian mechanics and the various theorems supposedly establishing the impossibility of hidden variables. In this section we wish to make several comments on this matter.

\subsection{Experiments and Random Variables}

In Bohmian mechanics we understand very naturally how random variables arise in association with experiments: the initial complete state $(Q, \Psi)$ of system and apparatus evolves deterministically and uniquely determines the outcome of the experiment; however, as the initial configuration $Q$ is in quantum equilibrium, the outcome of the experiment is random.

A general experiment $\mathscr{E}$ is then always associated a random variable (RV) $Z$ describing its result. In other words, according to Bohmian mechanics, there is a natural association

$$
\mathscr{E} \mapsto Z,
$$

between experiments and RVs. Moreover, whenever the statistics of the result of $\mathscr{E}$ is governed by a self-adjoint operator $A$ on the Hilbert space of the system, with the spectral measure of $A$ determining the distribution of $Z$, for which we shall write $Z \mapsto A$ (see (2.37)), Bohmian mechanics establishes thereby a natural association between $\mathscr{E}$ and $A$

$$
\mathscr{E} \mapsto A
$$


While for Bohmian mechanics the result $Z$ depends in general on both $X$ and $Y$, the initial configurations of the system and of the apparatus, for many real-world experiments $Z$ depends only on $X$ and the randomness in the result of the experiment is thus due solely to randomness in the initial configuration of the system alone. This is most obvious in the case of genuine position measurements (for which $Z(X, Y)=X$ ). That in fact the apparatus need not introduce any extra randomness for many other real-world experiments as well follows then from the observation that the role of the apparatus in many real-world experiments is to provide suitable background fields, which introduce no randomness, as well as a final detection, a measurement of the actual positions of the particles of the system. In particular, this is the case for those experiments most relevant to the issue of hidden variables, such as Stern-Gerlach measurements of spin, as well as for momentum measurements and more generally scattering experiments, which are completed by a final detection of position.

The result of these experiments is then given by a random variable

$$
Z=F\left(X_{T}\right)=G(X)
$$

where $T$ is the final time of the experiment, ${ }^{40}$ on the probability space $\{\Omega, \mathbb{P}\}$, where $\Omega=\{X\}$ is the set of initial configurations of the system and $\mathbb{P}(d x)=|\psi|^{2} d x$ is the quantum equilibrium distribution associated with the initial wave function $\psi$ of the system. For these experiments (see Section 5.4) the distribution of $Z$ is always governed by a PVM, corresponding to some self-adjoint operator $A, Z \mapsto A$, and thus Bohmian mechanics provides in these cases a natural $\operatorname{map} \mathscr{E} \mapsto A$.

\subsection{Random Variables, Operators, and the Impossibility Theorems}

We would like to briefly review the status of the so-called impossibility theorems for hidden variables, the most famous of which are due to von Neumann [74, Gleason [41, Kochen and Specker [53], and Bell [5]. Since Bohmian mechanics exists, these theorems can't possibly establish the impossibility of hidden variables, the widespread belief to the contrary notwithstanding. What these theorems do establish, in great generality, is that there is no "good" map from selfadjoint operators on a Hilbert space $\mathcal{H}$ to random variables on a common probability space,

$$
A \mapsto Z \equiv Z_{A}
$$

where $Z_{A}=Z_{A}(\lambda)$ should be thought of as the result of "measuring $A$ " when the hidden variables, that complete the quantum description and restore determinism, have value $\lambda$. Different senses of "good" correspond to different impossibility theorems.

For any particular choice of $\lambda$, say $\lambda_{0}$, the map (8.10) is transformed to a value map

$$
A \mapsto v(A)
$$

\footnotetext{
${ }^{40}$ Concerning the most common of all real-world quantum experiments, scattering experiments, although they are completed by a final detection of position, this detection usually occurs, not at a definite time $T$, but at a random time, for example when a particle enters a localized detector. Nonetheless, for computational purposes the final detection can be regarded as taking place at a definite time $T$. This is a consequence of the flux-acrosssurfaces theorem [19, 26, 27, which establishes an asymptotic equivalence between flux across surfaces (detection at a random time) and scattering into cones (detection at a definite time).
} 
from self-adjoint operators to real numbers (with $v(A)=Z_{A}\left(\lambda_{0}\right)$ ). The stronger impossibility theorems establish the impossibility of a good value map, again with different senses of "good" corresponding to different theorems.

Note that such theorems are not very surprising. One would not expect there to be a "good" map from a noncommutative algebra to a commutative one.

One of von Neumann's assumptions was, in effect, that the map (8.10) be linear. While mathematically natural, this assumption is physically rather unreasonable and in any case is entirely unnecessary. In order to establish that there is no good map (8.10), it is sufficient to require that the map be good in the minimal sense that the following agreement condition is satisfied:

Whenever the quantum mechanical joint distribution of a set of self-adjoint operators $\left(A_{1}, \ldots, A_{m}\right)$ exists, i.e., when they form a commuting family, the joint distribution of the corresponding set of random variables, i.e., of $\left(Z_{A_{1}}, \ldots, Z_{A_{m}}\right)$, agrees with the quantum mechanical joint distribution.

The agreement condition implies that all deterministic relationships among commuting observables must be obeyed by the corresponding random variables. For example, if $A, B$ and $C$ form a commuting family and $C=A B$, then we must have that $Z_{C}=Z_{A} Z_{B}$ since the joint distribution of $Z_{A}, Z_{B}$ and $Z_{C}$ must assign probability 0 to the set $\left\{(a, b, c) \in \mathbb{R}^{3} \mid c \neq a b\right\}$. This leads to a minimal condition for a good value map $A \mapsto v(A)$, namely that it preserve functional relationships among commuting observables: For any commuting family $A_{1}, \ldots, A_{m}$, whenever $f\left(A_{1}, \ldots, A_{m}\right)=0$ (where $f: \mathbb{R}^{m} \rightarrow \mathbb{R}$ represents a linear, multiplicative, or any other relationship among the $A_{i}$ 's), the corresponding values must satisfy the same relationship, $f\left(v\left(A_{1}\right), \ldots, v\left(A_{m}\right)\right)=0$.

The various impossibility theorems correctly demonstrate that there are no maps, from selfadjoint operators to random variables or to values, that are good, merely in the minimal senses described above. ${ }^{41}$

We note that while the original proofs of the impossibility of a good value map, in particular that of the Kochen-Specker theorem, were quite involved, in more recent years drastically simpler proofs have been found (for example, by Peres [67, by Greenberg, Horne, and Zeilinger [45, and by Mermin [62]).

In essence, one establishes the impossibility of a good map $A \mapsto Z_{A}$ or $A \mapsto v(A)$ by showing that the $v(A)$ 's, or $Z_{A}$ 's, would have to satisfy impossible relationships. These impossible relationships are very much like the following: $Z_{A}=Z_{B}=Z_{C} \neq Z_{A}$. However no impossible relationship can arise for only three quantum observables, since they would have to form a commuting family, for which quantum mechanics would supply a joint probability distribution. Thus the quantum relationships can't possibly lead to an inconsistency for the values of the random variables in this case.

With four observables $A, B, C$, and $D$ it may easily happen that $[A, B]=0,[B, C]=0$, $[C, D]=0$, and $[D, A]=0$ even though they don't form a commuting family (because, say, $[A, C] \neq 0)$. It turns out, in fact, that four observables suffice for the derivation of impossible

\footnotetext{
${ }^{41}$ Another natural sense of good map $A \mapsto v(A)$ is given by the requirement that $v(\mathbf{A}) \in \operatorname{sp}(\mathbf{A})$, where $\mathbf{A}=\left(A_{1}, \ldots, A_{m}\right)$ is a commuting family, $v(\mathbf{A})=\left(v\left(A_{1}\right), \ldots, v\left(A_{m}\right)\right) \in \mathbb{R}^{m}$ and $\mathrm{sp}(\mathbf{A})$ is the joint spectrum of the family. That a map good in this sense is impossible follows from the fact that if $\alpha=\left(\alpha_{1}, \ldots \alpha_{m}\right) \in \operatorname{sp}(\mathbf{A})$, then $\alpha_{1}, \ldots \alpha_{m}$ must obey all functional relationships for $A_{1}, \ldots, A_{m}$.
} 
quantum relationships. Perhaps the simplest example of this sort is due to Hardy [48, who showed that for almost every quantum state for two spin $1 / 2$ particles there are four observables $A, B, C$, and $D$ (two of which happen to be spin components for one of the particles while the other two are spin components for the other particle) whose quantum mechanical pair-wise distributions for commuting pairs are such that a good map to random variables must yield random variables $Z_{A}, Z_{B}, Z_{C}$, and $Z_{D}$ obeying the following relationships:

(1) The event $\left\{Z_{A}=1\right.$ and $\left.Z_{B}=1\right\}$ has positive probability (with an optimal choice of the quantum state, about .09).

(2) If $\left\{Z_{A}=1\right\}$ then $\left\{Z_{D}=1\right\}$.

(3) If $\left\{Z_{B}=1\right\}$ then $\left\{Z_{C}=1\right\}$.

(4) The event $\left\{Z_{D}=1\right.$ and $\left.Z_{C}=1\right\}$ has probability 0 .

Clearly, there exist no such random variables.

The point we wish to emphasize here, however, is that although they are correct and although their hypotheses may seem minimal, these theorems are nonetheless far less relevant to the possibility of a deterministic completion of quantum theory than one might imagine. In the next subsection we will elaborate on how that can be so. We shall explain why we believe such theorems have little physical significance for the issues of determinism and hidden variables. We will separately comment later in this section on Bell's related nonlocality analysis [5], which does have profound physical implications.

\subsection{Contextuality}

It is a simple fact there can be no map $A \mapsto Z_{A}$, from self-adjoint operators on $\mathcal{H}$ (with $\operatorname{dim}(\mathcal{H}) \geq$ 3 ) to random variables on a common probability space, that is good in the minimal sense that the joint probability distributions for the random variables agree with the corresponding quantum mechanical distributions, whenever the latter ones are defined. But does not Bohmian mechanics yield precisely such a map? After all, have we not emphasized how Bohmian mechanics naturally associates with any experiment a random variable $Z$ giving its result, in a manner that is in complete agreement with the quantum mechanical predictions for the result of the experiment? Given a quantum observable $A$, let $Z_{A}$ be then the result of a measurement of $A$. What gives?

Before presenting what we believe to be the correct response, we mention some possible responses that are off-target. It might be objected that measurements of different observables will involve different apparatuses and hence different probability spaces. However, one can simultaneously embed all the relevant probability spaces into a huge common probability space. It might also be objected that not all self-adjoint operators can be realistically be measured. But to arrive at inconsistency one need consider, as mentioned in the last subsection, only 4 observables, each of which are spin components and are thus certainly measurable, via Stern-Gerlach experiments. Thus, in fact, no enlargement of probability spaces need be considered to arrive at a contradiction, since as we emphasized at the end of Section 8.1 the random variables giving the results of Stern-Gerlach experiments are functions of initial particle positions, so that for joint measurements of pairs of spin components for 2-particles the corresponding results are random variables on the common probability space of initial configurations of the 2 particles, equipped with the quantum equilibrium distribution determined by the initial wave function. 
There must be a mistake. But where could it be? The mistake occurs, in fact, so early that it is difficult to notice it. It occurs at square one. The difficulty lies not so much in any conditions on the map $A \mapsto Z_{A}$, but in the conclusion that Bohmian mechanics supplies such a map at all.

What Bohmian mechanics naturally supplies is a map $\mathscr{E} \mapsto Z_{\mathscr{E}}$, from experiments to random variables. When $Z_{\mathscr{E}} \mapsto A$, so that we speak of $\mathscr{E}$ as a measurement of $A(\mathscr{E} \mapsto A)$, this very language suggests that insofar as the random variable is concerned all that matters is that $\mathscr{E}$ measures $A$, and the map $\mathscr{E} \mapsto Z_{\mathscr{E}}$ becomes a map $A \mapsto Z_{A}$. After all, if $\mathscr{E}$ were a genuine measurement of $A$, revealing, that is, the preexisting (i.e., prior to the experiment) value of the observable $A$, then $Z$ would have to agree with that value and hence would be an unambiguous random variable depending only on $A$.

But this sort of argument makes sense only if we take the quantum talk of operators as observables too seriously. We have emphasized in this paper that operators do naturally arise in association with quantum experiments. But there is little if anything in this association, beyond the unfortunate language that is usually used to describe it, that supports the notion that the operator $A$ associated with an experiment $\mathscr{E}$ is in any meaningful way genuinely measured by the experiment. From the nature of the association itself, it is difficult to imagine what this could possibly mean. And for those who think they imagine some meaning in this talk, the impossibility theorems show they are mistaken.

The bottom line is this: in Bohmian mechanics the random variables $Z_{\mathscr{E}}$ giving the results of experiments $\mathscr{E}$ depend, of course, on the experiment, and there is no reason that this should not be the case when the experiments under consideration happen to be associated with the same operator. Thus with any self-adjoint operator $A$, Bohmian mechanics naturally may associate many different random variables $Z_{\mathscr{E}}$, one for each different experiment $\mathscr{E} \mapsto A$ associated with $A$. A crucial point here is that the map $\mathscr{E} \mapsto A$ is many-to-one. ${ }^{42}$

Suppose we define a map $A \mapsto Z_{A}$ by selecting, for each $A$, one of the experiments, call it $\mathscr{E}_{A}$, with which $A$ is associated, and define $Z_{A}$ to be $Z_{\mathscr{E}_{A}}$. Then the map so defined can't be good, because of the impossibility theorems; moreover there is no reason to have expected the map to be good. Suppose, for example, that $[A, B]=0$. Should we expect that the joint distribution of $Z_{A}$ and $Z_{B}$ will agree with the joint quantum mechanical distribution of $A$ and $B$ ? Only if the experiments $\mathscr{E}_{A}$ and $\mathscr{E}_{B}$ used to define $Z_{A}$ and $Z_{B}$ both involved a common experiment that "simultaneously measures $A$ and $B$," i.e., an experiment that is associated with the commuting family $(A, B)$. If we consider now a third operator $C$ such that $[A, C]=0$, but $[B, C] \neq 0$, then there is no choice of experiment $\mathscr{E}$ that would permit the definition of a random variable $Z_{A}$ relevant both to a "simultaneous measurement of $A$ and $B$ " and a "simultaneous measurement of $A$ and $C$ " since no experiment is a "simultaneous measurement of $A, B$, and $C$." In the situation just described we must consider at least two random variables associated with $A, Z_{A, B}$ and $Z_{A, C}$, depending upon whether we are considering an experiment "measuring $A$ and $B$ " or an experiment "measuring $A$ and $C$." It should be clear that when the random variables are assigned to experiments in this way, the possibility of conflict with the predictions of orthodox quantum theory is eliminated. It should also be clear, in view of what we have

\footnotetext{
${ }^{42}$ We wish to remark that, quite aside from this many-to-oneness, the random variables $Z \mathscr{E}$ cannot generally be regarded as corresponding to any sort of natural property of the "measured" system. $Z_{\mathscr{E}}$, in general a function of the initial configuration of the system-apparatus composite, may fail to be a function of the configuration of the system alone. And even when, as is often the case, $Z_{\mathscr{E}}$ does depend only on the initial configuration of the system, owing to chaotic dynamics this dependence could have an extremely complex character.
} 
repeatedly stressed, that quite aside from the impossibility theorems, this way of associating random variables with experiments is precisely what emerges in Bohmian mechanics.

The dependence of the result of a "measurement of the observable $A$ " upon the other observables, if any, that are "measured simultaneously together with $A$ " - e.g., that $Z_{A, B}$ and $Z_{A, C}$ may be different - is called contextuality: the result of an experiment depends not just on "what observable the experiment measures" but on more detailed information that conveys the "context" of the experiment. The essential idea, however, if we avoid misleading language, is rather trivial: that the result of an experiment depends on the experiment.

To underline this triviality we remark that for two experiments, $\mathscr{E}$ and $\mathscr{E}$, that "measure $A$ and only $A$ " and involve no simultaneous "measurement of another observable," the results

$Z_{\mathscr{E}}$ and $Z_{\mathscr{E}}$ may disagree. For example in Section [7.5 we described experiments $\mathscr{E}$ and $\mathscr{E}^{\prime}$ both of which "measured the position operator" but only one of which measured the actual initial position of the relevant particle, so that for these experiments in general $Z_{\mathscr{E}} \neq Z_{\mathscr{E}}$.

One might feel, however, that in the example just described the experiment that does not measure the actual position is somewhat disreputable - even though it is in fact a "measurement of the position operator." We shall therefore give another example, due to D. Albert [2, in which the experiments are as simple and canonical as possible and are entirely on the same footing. Let $\mathscr{E} \uparrow$ and $\mathscr{E} \downarrow$ be Stern-Gerlach measurements of $A=\sigma_{z}$, with $\mathscr{E}_{\downarrow}$ differing from $\mathscr{E} \uparrow$ only in that the polarity of the Stern-Gerlach magnet for $\mathscr{E}_{\downarrow}$ is the reverse of that for $\mathscr{E} \uparrow$. (In particular, the geometry of the magnets for $\mathscr{E} \uparrow$ and $\mathscr{E} \downarrow$ is the same.) If the initial wave function $\psi_{\text {symm }}$ and the magnetic field $\pm B$ have sufficient reflection symmetry with respect to a plane between the poles of the Stern-Gerlach magnets, the particle whose spin component is being "measured" cannot cross this plane of symmetry, so that if the particle is initially above, respectively below, the symmetry plane, it will remain above, respectively below, that plane. But because their magnets have opposite polarity, $\mathscr{E}_{\uparrow}$ and $\mathscr{E}_{\downarrow}$ involve opposite calibrations: $F_{\uparrow}=-F_{\downarrow}$. It follows that

$$
Z_{\mathscr{E} \uparrow}^{\psi_{\text {symm }}}=-Z_{\mathscr{E}}^{\psi_{\text {symm }}}
$$

and the two experiments completely disagree about the "value of $\sigma_{z}$ " in this case.

The essential point illustrated by the previous example is that instead of having in Bohmian mechanics a natural association $\sigma_{z} \mapsto Z_{\sigma_{z}}$, we have a rather different pattern of relationships, given in the example by

$$
\begin{aligned}
& \mathscr{E}_{\uparrow} \rightarrow Z_{\mathscr{E}_{\uparrow}} \searrow \sigma_{z}, \\
& \mathscr{E}_{\downarrow} \rightarrow Z_{\mathscr{E}_{\downarrow}},
\end{aligned}
$$

\subsection{Against "Contextuality"}

The impossibility theorems require the assumption of noncontextuality, that the random variable $Z$ giving the result of a "measurement of quantum observable $A$ " should depend on $A$ alone, further experimental details being irrelevant. How big a deal is contextuality, the violation of this assumption? Here are two ways of describing the situation:

1. In quantum mechanics (or quantum mechanics supplemented with hidden variables), observables and properties have a novel, highly nonclassical aspect: they (or the result of measuring them) depend upon which other compatible properties, if any, are measured together with them.

In this spirit, Bohm and Hiley [16] write that (page 109) 
the quantum properties imply ... that measured properties are not intrinsic but are inseparably related to the apparatus. It follows that the customary language that attributes the results of measurements ... to the observed system alone can cause confusion, unless it is understood that these properties are actually dependent on the total relevant context.

They later add that (page 122)

The context dependence of results of measurements is a further indication of how our interpretation does not imply a simple return to the basic principles of classical physics. It also embodies, in a certain sense, Bohr's notion of the indivisibility of the combined system of observing apparatus and observed object.

2. The result of an experiment depends upon the experiment. Or, as expressed by Bell [10] (pg.166),

A final moral concerns terminology. Why did such serious people take so seriously axioms which now seem so arbitrary? I suspect that they were misled by the pernicious misuse of the word 'measurement' in contemporary theory. This word very strongly suggests the ascertaining of some preexisting property of some thing, any instrument involved playing a purely passive role. Quantum experiments are just not like that, as we learned especially from Bohr. The results have to be regarded as the joint product of 'system' and 'apparatus,' the complete experimental set-up. But the misuse of the word 'measurement' makes it easy to forget this and then to expect that the 'results of measurements' should obey some simple logic in which the apparatus is not mentioned. The resulting difficulties soon show that any such logic is not ordinary logic. It is my impression that the whole vast subject of 'Quantum Logic' has arisen in this way from the misuse of a word. I am convinced that the word 'measurement' has now been so abused that the field would be significantly advanced by banning its use altogether, in favour for example of the word 'experiment.'

With one caveat, we entirely agree with Bell's observation. The caveat is this: We do not believe that the difference between quantum mechanics and classical mechanics is quite as crucial for Bell's moral as his language suggests it is. For any experiment, quantum or classical, it would be a mistake to regard any instrument involved as playing a purely passive role, unless the experiment is a genuine measurement of a property of a system, in which case the result is determined by the initial conditions of the system alone. However, a relevant difference between classical and quantum theory remains: Classically it is usually taken for granted that it is in principle possible to measure any observable without seriously affecting the observed system, which is clearly false in quantum mechanics (or Bohmian mechanics). ${ }^{43}$

Mermin has raised a similar question 62] (pg. 811):

Is noncontextuality, as Bell seemed to suggest, as silly a condition as von Neumann's ...?

To this he answers:

\footnotetext{
${ }^{43}$ The assumption could (and probably should) also be questioned classically.
} 
I would not characterize the assumption of noncontextuality as a silly constraint on a hidden-variables theory. It is surely an important fact that the impossibility of embedding quantum mechanics in a noncontextual hidden-variables theory rests not only on Bohr's doctrine of the inseparability of the objects and the measuring instruments, but also on a straightforward contradiction, independent of one's philosophic point of view, between some quantitative consequences of noncontextuality and the quantitative predictions of quantum mechanics.

This is a somewhat strange answer. First of all, it applies to von Neumann's assumption (linearity), which Mermin seems to agree is silly, as well as to the assumption of noncontextuality. And the statement has a rather question-begging flavor, since the importance of the fact to which Mermin refers would seem to depend on the nonsilliness of the assumption which the fact concerns.

Be that as it may, Mermin immediately supplies his real argument for the nonsilliness of noncontextuality. Concerning two experiments for "measuring observable $A$," he writes that

it is ... an elementary theorem of quantum mechanics that the joint distribution ... for the first experiment yields precisely the same marginal distribution (for $A$ ) as does the joint distribution ... for the second, in spite of the different experimental arrangements. ... The obvious way to account for this, particularly when entertaining the possibility of a hidden-variables theory, is to propose that both experiments reveal a set of values for $A$ in the individual systems that is the same, regardless of which experiment we choose to extract them from. ... A contextual hidden-variables account of this fact would be as mysteriously silent as the quantum theory on the question of why nature should conspire to arrange for the marginal distributions to be the same for the two different experimental arrangements.

A bit later, Mermin refers to the "striking insensitivity of the distribution to changes in the experimental arrangement."

For Mermin there is a mystery, something that demands an explanation. It seems to us, however, that the mystery here is very much in the eye of the beholder. It is first of all somewhat odd that Mermin speaks of the mysterious silence of quantum theory concerning a question whose answer, in fact, emerges as an "elementary theorem of quantum mechanics." What better way is there to answer questions about nature than to appeal to our best physical theories?

More importantly, the "two different experimental arrangements," say $\mathscr{E}_{1}$ and $\mathscr{E}_{2}$, considered by Mermin are not merely any two randomly chosen experimental arrangements. They obviously must have something in common. This is that they are both associated with the same self-adjoint operator $A$ in the manner we have described: $\mathscr{E}_{1} \mapsto A$ and $\mathscr{E}_{2} \mapsto A$. It is quite standard to say in this situation that both $\mathscr{E}_{1}$ and $\mathscr{E}_{2}$ measure the observable $A$, but both for Bohmian mechanics and for orthodox quantum theory the very meaning of the association with the operator $A$ is merely that the distribution of the result of the experiment is given by the spectral measures for $A$. Thus there is no mystery in the fact that $\mathscr{E}_{1}$ and $\mathscr{E}_{2}$ have results governed by the same distribution, since, when all is said and done, it is on this basis, and this basis alone, that we are comparing them.

(One might wonder how it could be possible that there are two different experiments that are related in this way. This is a somewhat technical question, rather different from Mermin's, and it is one that Bohmian mechanics and quantum mechanics readily answer, as we have explained in this paper. In this regard it would probably be good to reflect further on the simplest example of such experiments, the Stern-Gerlach experiments $\mathscr{E}_{\uparrow}$ and $\mathscr{E}_{\downarrow}$ discussed in the previous subsection.) 
It is also difficult to see how Mermin's proposed resolution of the mystery, "that both experiments reveal a set of values for $A \ldots$ that is the same, regardless of which experiment we choose to extract them from," could do much good. He is faced with a certain pattern of results in two experiments that would be explained if the experiments did in fact genuinely measure the same thing. The experiments, however, as far as any detailed quantum mechanical analysis of them is concerned, don't appear to be genuine measurements of anything at all. He then suggests that the mystery would be resolved if, indeed, the experiments did measure the same thing, the analysis to the contrary notwithstanding. But this proposal merely replaces the original mystery with a bigger one, namely, of how the experiments could in fact be understood as measuring the same thing, or anything at all for that matter. It is like explaining the mystery of a talking cat by saying that the cat is in fact a human being, appearances to the contrary notwithstanding.

A final complaint about contextuality: the terminology is misleading. It fails to convey with sufficient force the rather definitive character of what it entails: "Properties" that are merely contextual are not properties at all; they do not exist, and their failure to do so is in the strongest sense possible!

\subsection{Nonlocality, Contextuality and Hidden Variables}

There is, however, a situation where contextuality is physically relevant. Consider the EPRB experiment, outlined at the end of Section 3.6. In this case the dependence of the result of a measurement of the spin component $\boldsymbol{\sigma}_{1} \cdot \mathbf{a}$ of a particle upon which spin component of a distant particle is measured together with it - the difference between $Z_{\boldsymbol{\sigma}_{1} \cdot \mathbf{a}, \boldsymbol{\sigma}_{2} \cdot \mathbf{b}}$ and $Z_{\boldsymbol{\sigma}_{1} \cdot \mathbf{a}, \boldsymbol{\sigma}_{2} \cdot \mathbf{c}}$ (using the notation described in the seventh paragraph of Section 8.3) - is an expression of nonlocality, of, in Einstein words, a "spooky action at distance." More generally, whenever the relevant context is distant, contextuality implies nonlocality.

Nonlocality is an essential feature of Bohmian mechanics: the velocity, as expressed in the guiding equation (2.2), of any one of the particles of a many-particle system will typically depend upon the positions of the other, possibly distant, particles whenever the wave function of the system is entangled, i.e., not a product of single-particle wave functions. In particular, this is true for the EPRB experiment under examination. Consider the extension of the single particle Hamiltonian (2.12) to the two-particle case, namely

$$
H=-\frac{\hbar^{2}}{2 m_{1}} \nabla_{1}^{2}-\frac{\hbar^{2}}{2 m_{2}} \nabla_{2}^{2}-\mu_{1} \boldsymbol{\sigma}_{1} \cdot \mathbf{B}\left(\mathbf{x}_{\mathbf{1}}\right)-\mu_{2} \boldsymbol{\sigma}_{2} \cdot \mathbf{B}\left(\mathbf{x}_{\mathbf{2}}\right) .
$$

Then for initial singlet state, and spin measurements as described in Sections 2.5] and 5.2. it easily follows from the laws of motion of Bohmian mechanics that

$$
Z_{\boldsymbol{\sigma}_{1} \cdot \mathbf{a}, \boldsymbol{\sigma}_{2} \cdot \mathbf{b}} \neq Z_{\boldsymbol{\sigma}_{1} \cdot \mathbf{a}, \boldsymbol{\sigma}_{2} \cdot \mathbf{c}}
$$

This was observed long ago by Bell [6]. In fact, Bell's examination of Bohmian mechanics led him to his celebrated nonlocality analysis. In the course of his investigation of Bohmian mechanics he observed that ([10], p. 11)

in this theory an explicit causal mechanism exists whereby the disposition of one piece of apparatus affects the results obtained with a distant piece. 
Bohm of course was well aware of these features of his scheme, and has given them much attention. However, it must be stressed that, to the present writer's knowledge, there is no proof that any hidden variable account of quantum mechanics must have this extraordinary character. It would therefore be interesting, perhaps, to pursue some further "impossibility proofs," replacing the arbitrary axioms objected to above by some condition of locality, or of separability of distant systems.

In a footnote, Bell added that "Since the completion of this paper such a proof has been found." This proof was published in his 1964 paper [5], "On the Einstein-Podolsky-Rosen Paradox," in which he derives Bell's inequality, the basis of his conclusion of quantum nonlocality.

We find it worthwhile to reproduce here the analysis of Bell, deriving a simple inequality equivalent to Bell's, in order to highlight the conceptual significance of Bell's analysis and, at the same time, its mathematical triviality. The analysis involves two parts. The first part, the Einstein-Podolsky-Rosen argument applied to the EPRB experiment, amounts to the observation that for the singlet state the assumption of locality implies the existence of noncontextual hidden variables. More precisely, it implies, for the singlet state, the existence of random variables $Z_{\boldsymbol{\alpha}}^{i}=Z_{\boldsymbol{\alpha} \cdot \boldsymbol{\sigma}_{i}}, i=1,2$, corresponding to all possible spin components of the two particles, that obey the agreement condition described in Section 8.2. In particular, focusing on components in only 3 directions $\mathbf{a}, \mathbf{b}$ and $\mathbf{c}$ for each particle, locality implies the existence of 6 random variables

$$
Z_{\boldsymbol{\alpha}}^{i} \quad i=1,2 \quad \boldsymbol{\alpha}=\mathbf{a}, \mathbf{b}, \mathbf{c}
$$

such that

$$
\begin{aligned}
& Z_{\boldsymbol{\alpha}}^{i}= \pm 1 \\
& Z_{\boldsymbol{\alpha}}^{1}=-Z_{\boldsymbol{\alpha}}^{2}
\end{aligned}
$$

and, more generally,

$$
\operatorname{Prob}\left(Z_{\boldsymbol{\alpha}}^{1} \neq Z_{\boldsymbol{\alpha}}^{2}\right)=q_{\boldsymbol{\alpha} \boldsymbol{\beta}}
$$

the corresponding quantum mechanical probabilities. This conclusion amounts to the idea that measurements of the spin components reveal preexisting values (the $Z_{\boldsymbol{\alpha}}^{i}$ ), which, assuming locality, is implied by the perfect quantum mechanical anticorrelations [5]:

Now we make the hypothesis, and it seems one at least worth considering, that if the two measurements are made at places remote from one another the orientation of one magnet does not influence the result obtained with the other. Since we can predict in advance the result of measuring any chosen component of $\boldsymbol{\sigma}_{2}$, by previously measuring the same component of $\boldsymbol{\sigma}_{1}$, it follows that the result of any such measurement must actually be predetermined.

People very often fail to appreciate that the existence of such variables, given locality, is not an assumption but a consequence of Bell's analysis. Bell repeatedly stressed this point (by determinism Bell here means the existence of hidden variables):

It is important to note that to the limited degree to which determinism plays a role in the EPR argument, it is not assumed but inferred. What is held sacred is the principle of 'local causality' - or 'no action at a distance'. ...

It is remarkably difficult to get this point across, that determinism is not a presupposition of the analysis. ([10], p. 143)

Despite my insistence that the determinism was inferred rather than assumed, you might still suspect somehow that it is a preoccupation with determinism that creates 
the problem. Note well then that the following argument makes no mention whatever of determinism. ... Finally you might suspect that the very notion of particle, and particle orbit ... has somehow led us astray. ... So the following argument will not mention particles, nor indeed fields, nor any other particular picture of what goes on at the microscopic level. Nor will it involve any use of the words 'quantum mechanical system', which can have an unfortunate effect on the discussion. The difficulty is not created by any such picture or any such terminology. It is created by the predictions about the correlations in the visible outputs of certain conceivable experimental set-ups. ([10], p. 150)

The second part of the analysis, which unfolds the "difficulty ... created by the ... correlations," involves only very elementary mathematics. Clearly,

$$
\operatorname{Prob}\left(\left\{Z_{\mathbf{a}}^{1}=Z_{\mathbf{b}}^{1}\right\} \cup\left\{Z_{\mathbf{b}}^{1}=Z_{\mathbf{c}}^{1}\right\} \cup\left\{Z_{\mathbf{c}}^{1}=Z_{\mathbf{a}}^{1}\right\}\right)=1 .
$$

since at least two of the three (2-valued) variables $Z_{\boldsymbol{\alpha}}^{1}$ must have the same value. Hence, by elementary probability theory,

$$
\operatorname{Prob}\left(Z_{\mathbf{a}}^{1}=Z_{\mathbf{b}}^{1}\right)+\operatorname{Prob}\left(Z_{\mathbf{b}}^{1}=Z_{\mathbf{c}}^{1}\right)+\operatorname{Prob}\left(Z_{\mathbf{c}}^{1}=Z_{\mathbf{a}}^{1}\right) \geq 1,
$$

and using the perfect anticorrelations (8.13) we have that

$$
\operatorname{Prob}\left(Z_{\mathbf{a}}^{1}=-Z_{\mathbf{b}}^{2}\right)+\operatorname{Prob}\left(Z_{\mathbf{b}}^{1}=-Z_{\mathbf{c}}^{2}\right)+\operatorname{Prob}\left(Z_{\mathbf{c}}^{1}=-Z_{\mathbf{a}}^{2}\right) \geq 1,
$$

which is equivalent to Bell's inequality and in conflict with (8.14). For example, when the angles between $\mathbf{a}, \mathbf{b}$ and $\mathbf{c}$ are $120^{\circ}$ the 3 relevant quantum correlations $q_{\boldsymbol{\alpha} \boldsymbol{\beta}}$ are all $1 / 4$.

To summarize the argument, let $\mathrm{H}$ be the hypothesis of the existence of the noncontextual hidden variables we have described above. Then the logic of the argument is:

$$
\begin{array}{rcll}
\text { Part 1: } & \text { quantum mechanics }+ \text { locality } & \Rightarrow \mathrm{H} \\
\text { Part 2: } & \text { quantum mechanics } & \Rightarrow \text { not } \mathrm{H} \\
\text { Conclusion: } & \text { quantum mechanics } & \Rightarrow \text { not locality }
\end{array}
$$

To fully grasp the argument it is important to appreciate that the identity of $\mathrm{H}$ - the existence of the noncontextual hidden variables - is of little substantive importance. What is important is not so much the identity of $\mathrm{H}$ as the fact that $\mathrm{H}$ is incompatible with the predictions of quantum theory. The identity of $\mathrm{H}$ is, however, of great historical significance: It is responsible for the misconception that Bell proved that hidden variables are impossible, a belief until recently almost universally shared by physicists.

Such a misconception has not been the only reaction to Bell's analysis. Roughly speaking, we may group the different reactions into three main categories, summarized by the following statements:

1. Hidden variables are impossible.

2. Hidden variables are possible, but they must be contextual.

3. Hidden variables are possible, but they must be nonlocal. 
Statement 1 is plainly wrong. Statement 2 is correct but not terribly significant. Statement 3 is correct, significant, but nonetheless rather misleading. It follow from (8.16) and (8.17) that any account of quantum phenomena must be nonlocal, not just any hidden variables account. Bell's argument shows that nonlocality is implied by the predictions of standard quantum theory itself. Thus if nature is governed by these predictions, then nature is nonlocal. (That nature is so governed, even in the crucial EPR-correlation experiments, has by now been established by a great many experiments, the most conclusive of which is perhaps that of Aspect [4.)

\section{Against Naive Realism About Operators}

Traditional naive realism is the view that the world is pretty much the way it seems, populated by objects which force themselves upon our attention as, and which in fact are, the locus of sensual qualities. A naive realist regards these "secondary qualities," for example color, as objective, as out there in the world, much as perceived. A decisive difficulty with this view is that once we understand, say, how our perception of what we call color arises, in terms of the interaction of light with matter, and the processing of the light by the eye, and so on, we realize that the presence out there of color per se would play no role whatsoever in these processes, that is, in our understanding what is relevant to our perception of "color." At the same time, we may also come to realize that there is, in the description of an object provided by the scientific world-view, as represented say by classical physics, nothing which is genuinely "color-like."

A basic problem with quantum theory, more fundamental than the measurement problem and all the rest, is a naive realism about operators, a fallacy which we believe is far more serious than traditional naive realism: With the latter we are deluded partly by language but in the main by our senses, in a manner which can scarcely be avoided without a good deal of scientific or philosophical sophistication; with the former we are seduced by language alone, to accept a view which can scarcely be taken seriously without a large measure of (what often passes for) sophistication.

Not many physicists - or for that matter philosophers - have focused on the issue of naive realism about operators, but Schrödinger and Bell have expressed similar or related concerns:

... the new theory [quantum theory] ... considers the [classical] model suitable for guiding us as to just which measurements can in principle be made on the relevant natural object. ... Would it not be pre-established harmony of a peculiar sort if the classical-epoch researchers, those who, as we hear today, had no idea of what measuring truly is, had unwittingly gone on to give us as legacy a guidance scheme revealing just what is fundamentally measurable for instance about a hydrogen atom!? [72]

Here are some words which, however legitimate and necessary in application, have no place in a formulation with any pretension to physical precision: system; apparatus; environment; microscopic, macroscopic; reversible, irreversible; observable; information; measurement.

... The notions of "microscopic" and "macroscopic" defy precise definition. ... Einstein said that it is theory which decides what is "observable". I think he was right. ... "observation" is a complicated and theory-laden business. Then that notion should not appear in the formulation of fundamental theory. ...

On this list of bad words from good books, the worst of all is "measurement". It must have a section to itself. [1] 
We agree almost entirely with Bell here. We insist, however, that "observable" is just as bad as "measurement," maybe even a little worse. Be that as it may, after listing Dirac's measurement postulates Bell continues:

It would seem that the theory is exclusively concerned about "results of measurement", and has nothing to say about anything else. What exactly qualifies some physical systems to play the role of "measurer"? Was the wave function of the world waiting to jump for thousands of millions of years until a single-celled living creature appeared? Or did it have to wait a little longer, for some better qualified system ... with a Ph.D.? If the theory is to apply to anything but highly idealized laboratory operations, are we not obliged to admit that more or less "measurement-like" processes are going on more or less all the time, more or less everywhere. Do we not have jumping then all the time?

The first charge against "measurement", in the fundamental axioms of quantum mechanics, is that it anchors the shifty split of the world into "system" and "apparatus". A second charge is that the word comes loaded with meaning from everyday life, meaning which is entirely inappropriate in the quantum context. When it is said that something is "measured" it is difficult not to think of the result as referring to some preexisting property of the object in question. This is to disregard Bohr's insistence that in quantum phenomena the apparatus as well as the system is essentially involved. If it were not so, how could we understand, for example, that "measurement" of a component of "angular momentum" ... in an arbitrarily chosen direction ... yields one of a discrete set of values? When one forgets the role of the apparatus, as the word "measurement" makes all too likely, one despairs of ordinary logic ...hence "quantum logic". When one remembers the role of the apparatus, ordinary logic is just fine.

In other contexts, physicists have been able to take words from ordinary language and use them as technical terms with no great harm done. Take for example the "strangeness", "charm", and "beauty" of elementary particle physics. No one is taken in by this "baby talk". ... Would that it were so with "measurement". But in fact the word has had such a damaging effect on the discussion, that I think it should now be banned altogether in quantum mechanics. (Ibid.)

While Bell focuses directly here on the misuse of the word "measurement" rather than on that of "observable," it is worth noting that the abuse of "measurement" is in a sense inseparable from that of "observable," i.e., from naive realism about operators. After all, one would not be very likely to speak of measurement unless one thought that something, some "observable" that is, was somehow there to be measured.

Operationalism, so often used without a full appreciation of its consequences, may lead many physicists to beliefs which are the opposite of what one might expect. Namely, by believing somehow that a physical property is and must be defined by an operational definition, many physicists come to regard properties such as spin and polarization, which can easily be operationally defined, as intrinsic properties of the system itself, the electron or photon, despite all the difficulties that this entails. If operational definitions were banished, and "real definitions" were required, there would be far less reason to regard these "properties" as intrinsic, since they are not defined in any sort of intrinsic way; in short, we have no idea what they really mean, and there is no reason to think they mean anything beyond the behavior exhibited by the system in interaction with an apparatus.

There are two primary sources of confusion, mystery and incoherence in the foundations of quantum mechanics: the insistence on the completeness of the description provided by the wave 
function, despite the dramatic difficulties entailed by this dogma, as illustrated most famously by the measurement problem; and naive realism about operators. While the second seems to point in the opposite direction from the first, the dogma of completeness is in fact nourished by naive realism about operators. This is because naive realism about operators tends to produce the belief that a more complete description is impossible because such a description should involve preexisting values of the quantum observables, values that are revealed by measurement. And this is impossible. But without naive realism about operators - without being misled by all the quantum talk of the measurement of observables - most of what is shown to be impossible by the impossibility theorems would never have been expected to begin with.

\section{Acknowledgments}

An early version of this paper had a fourth author: Martin Daumer. Martin left our group a long time ago and has not participated since in the very substantial changes in both form and content that the paper has undergone. His early contributions are very much appreciated. We thank Roderich Tumulka for a careful reading of this manuscript and helpful suggestions. This work was supported in part by NSF Grant No. DMS-9504556, by the DFG, and by the INFN. We are grateful for the hospitality that we have enjoyed, on more than one occasion, at the Mathematisches Institut of Ludwig-Maximilians-Universität München, at the Dipartimento di Fisica of Università degli Studi di Genova, and at the Mathematics Department of Rutgers University.

\section{References}

[1] Y. Aharonov, J. Anandan, and L. Vaidman. Meaning of the Wave Function. Physical Review A, 47: 4616-4626, 1993.

[2] D. Z. Albert. Quantum Mechanics and Experience. Cambridge, MA, Harvard University Press, 1992.

[3] S.T. Ali and G. G. Emch. Fuzzy Observables in Quantum Mechanics. Journal of Mathematical Physics, 15: 176-182, 1974.

[4] A. Aspect, J. Dalibard, and G. Roger. Experimental Test of Bell's Inequalities using TimeVarying Analyzers. Phys. Rev. Lett. 49: 1804-1807, 1982.

[5] J. S. Bell. On the Einstein-Podolsky-Rosen Paradox. Physics, 1: 195-200, 1964. Reprinted in [75], and in [10].

[6] J. S. Bell. On the Problem of Hidden Variables in Quantum Mechanics. Reviews of Modern Physics, 38: 447-452, 1966. Reprinted in [75] and in [10].

[7] J. S. Bell. De Broglie-Bohm, Delayed-Choice Double-Slit Experiment, and Density Matrix. International Journal of Quantum Chemistry: A Symposium, 14: 155-159, 1980. Reprinted in [10]. 
[8] J. S. Bell. Quantum Mechanics for Cosmologists. In Quantum Gravity 2, C. Isham, R. Penrose, and D. Sciama (eds.), Oxford University Press, New York, pp. 611-637. Reprinted in [10].

[9] J. S. Bell. On the Impossible Pilot Wave. Foundations of Physics, 12: 989-999, 1982. Reprinted in [10].

[10] J. S. Bell. Speakable and unspeakable in quantum mechanics. Cambridge University Press, Cambridge, 1987.

[11] J. S. Bell. Against "Measurement". Physics World, 3: 33-40, 1990. Also in 63].

[12] I. Bialynicki-Birula. On the Wave Function of the Photon. Acta Physica Polonica 86: 97-116, 1994.

[13] A. Böhm. Quantum Mechanics. Springer-Verlag, New York-Heidelberg-Berlin, 1979.

[14] D. Bohm. Quantum Theory. Prentice-Hall, Englewood Cliffs, N.J., 1951.

[15] D. Bohm. A Suggested Interpretation of the Quantum Theory in Terms of "Hidden" Variables: Parts I and II. Physical Review, 85: 166-193, 1952. Reprinted in [75].

[16] D. Bohm and B. J Hiley. The Undivided Universe: An Ontological Interpretation of Quantum Theory. London, Routledge \& Kegan Paul, 1993.

[17] N. Bohr. Atomic Physics and Human Knowledge. Wiley, New York, 1958.

[18] V. B. Braginsky, Y. I. Vorontsov, and K. S. Thorne. Quantum Nondemolition Measurements. Science, 209: 547-57, 1980. Reprinted in [75].

[19] M. Daumer, D. Dürr, S. Goldstein and N. Zanghì. On the Flux-Across-Surfaces Theorem, Lett. Math. Phys. 38: 103-116, 1996.

[20] M. Daumer, D. Dürr, S. Goldstein and N. Zanghì, On the Quantum Probability Flux Through Surfaces, J. Stat. Phys. 88: 967-977, 1997.

[21] E B. Davies. Quantum Theory of Open Systems. Academic Press, London-New York-San Francisco, 1976.

[22] C. Dewdney, L. Hardy, and E. J. Squires. How Late Meaurements of Quantum Trajectories Can Fool a Detector. Phys. Lett. A 184: 6-11, 1993.

[23] P. A. M. Dirac. The Principles of Quantum Mechanics. Oxford University Press, Oxford, 1930.

[24] D. Dürr, W. Fusseder, S. Goldstein, and N. Zanghì. Comment on: Surrealistic Bohm Trajectories. Z.f.Naturforsch., 48a: 1261-1262, 1993.

[25] D. Dürr, S. Goldstein, and N. Zanghì. Quantum Equilibrium and the Origin of Absolute Uncertainty. Journal of Statistical Physics, 67: 843-907, 1992. 
[26] D. Dürr, K. Münch-Berndl, and S. Teufel. The Flux Across Surfaces Theorem for Short Range Potentials without Energy Cutoffs. J. Math. Phys. 40: 1901-1922, 1999.

[27] D. Dürr, S. Goldstein, S. Teufel, and N. Zanghì. Scattering Theory from Microscopic First Principles. Physica A 279: 416-431, 2000.

[28] D. Dürr, S. Goldstein J. Taylor, and N. Zanghì. Bosons, Fermions, and the Topology of Configuration Space. In preparation.

[29] D. Dürr, S. Goldstein, R. Tumulka, and N. Zanghì. Quantum Hamiltonians and Stochastic Jumps. quant-ph/0303056.

[30] D. Dürr, S. Goldstein, R. Tumulka, and N. Zanghì. Trajectories and Particle Creation and Annihilation in Quantum Field Theory. J. Phys. A: Math. Gen. 36: 4143-4149, 2003.

[31] D. Dürr, S. Goldstein, R. Tumulka, and N. Zanghì. On the Role of Density Matrices in Bohmian Mechanics. In preparation.

[32] A. Einstein, B. Podolsky, and N. Rosen. Can Quantum-Mechanical Description of Physical Reality Be Considered Complete? Phys. Rev. 47: 777-780, 1935.

[33] E. Englert, M. O. Scully, G. Süssman, and H. Walther. Surrealistic Bohm Trajectories. Z. f. Naturforsch., 47a: 1175, 1992.

[34] R. P. Feynman and A. R. Hibbs. Quantum Mechanics and Path Integrals. McGraw-Hill, New York, 1965.

[35] M. Gell-Mann and J. B. Hartle. Quantum Mechanics in the Light of Quantum Cosmology. In W. Zurek, editor, Complexity, Entropy, and the Physics of Information, pages 425-458. Addison-Wesley, Reading, 1990. Also in [52].

[36] G. C. Ghirardi. Unpublished, 1981 (private communication).

[37] G. C. Ghirardi and T. Weber. Quantum Mechanics and Faster-Than-Light Communication: Methodological Considerations Nuovo Cimento 78B, 9, 1983.

[38] G. C. Ghirardi, A. Rimini, and T. Weber. Unified Dynamics for Microscopic and Macroscopic Systems. Physical Review D, 34: 470-491, 1986.

[39] G. C. Ghirardi and A. Rimini. Old and New Ideas in the Theory of Quantum Measurements. In 63.

[40] G. C. Ghirardi, P. Pearle, and A. Rimini. Markov Processes in Hilbert Space and Continous Spontaneous Localization of System of Identical Particles. Physical Review A, 42: 78-89, 1990.

[41] A. M. Gleason. Measures on the Closed Subspaces of a Hilbert Space. J. Math. and Mech. 6: 885-893, 1957

[42] S. Goldstein. Stochastic Mechanics and Quantum Theory. Journal of Statistical Physics, 47: 645-667, 1987. 
[43] S. Goldstein. Quantum Theory Without Observers. Part One: Physics Today, March 1998, 42-46. Part Two: Physics Today, April 1998, 38-42.

[44] S. Goldstein and D. Page. Linearly Positive Histories: Probabilities for a Robust Family of Sequences of Quantum Events. Phys. Rev. Lett. 74: 3715-3719, 1995.

[45] D. M. Greenberg, M. Horne, S. Shimony and A. Zeilenger. Bell's Theorem Without Inequalities. American Journal of Physics, 58: 1131-1143, 1990.

[46] R. B. Griffiths. Consistent Histories and the Interpretation of Quantum Mechanics. Journal of Statistical Physics, 36: 219-272, 1984.

[47] G. Grübl and K. Rheinberger. Time of Arrival from Bohmian Flow. J. Phys. A: Math. Gen. 35: 2907-2924, 2002.

[48] L. Hardy. Nonlocality for Two Particles Without Inequalities for Almost All Entangled States. Phys. Rev. Lett. 71: 1665, 1993.

[49] W. Heisenberg. Physics and Beyond; Encounters and Conversations. Harper \& Row, New York, 1971.

[50] A. S. Holevo. Probabilistic and Statistical Aspects of Quantum Theory, Volume 1 of NorthHolland Series in Statistics and Probability. North-Holland, Amsterdam-New York-Oxford, 1982.

[51] E. Joos and H. D. Zeh. The Emergence of Classical Properties through Interaction with the Environment. Zeitschrift für Physik B, 59: 223-243, 1985.

[52] S. Kobayashi, H. Ezawa, Y. Murayama, and S. Nomura, editors. Proceedings of the 3rd International Symposium on Quantum Mechanics in the Light of New Technology. Physical Society of Japan, 1990.

[53] S. Kochen and E. P. Specker. The Problem of Hidden Variables in Quantum Mechanics. Journal of Mathematics and Mechanics, 17:59-87, 1967.

[54] K. Kraus. Position Observables of the Photon, pp. 293-320 in W.C. Price and S.S. Chissick (eds.), The Uncertainty Principle and Foundations of Quantum Mechanics. New York: Wiley (1977)

[55] K. Kraus. States, Effects, and Operations. Lectures Notes in Physics 190, 1983.

[56] L. D. Landau and E. M. Lifshitz. Quantum Mechanics: Non-relativistic Theory. Pergamon Press, Oxford and New York, 1958. Translated from the Russian by J. B. Sykes and J. S. Bell.

[57] C. R. Leavens. Transmission, Reflection and Dwell Times within Bohm's Causal Interpretation of Quantum Mechanics. Solid State Communications, 74: 923, 1990.

[58] W. R. McKinnon and C. R. Leavens. Distributions of Delay Times and Transmission Times in Bohm's Causal Interpretation of Quantum Mechanics. Phys. Rev. A 51, 2748-2757, 1995. 
[59] C. R. Leavens. Time of Arrival in Quantum and Bohmian Mechanics. Phys. Rev. A 58: 840-847, 1998.

[60] A. J. Leggett. Macroscopic Quantum Systems and the Quantum Theory of Measurement. Supplement of the Progress of Theoretical Physics, 69: 80-100, 1980.

[61] G. Lüders. Über die Zustandsänderung durch den Messprozess. Annalen der Physik, 8: 322-328, 1951.

[62] D. N. Mermin. Hidden Variables and the Two Theorems of John Bell. Review of Modern Physics 65: 803-815, 1993.

[63] A. I. Miller, editor. Sixty-Two Years of Uncertainty: Historical, Philosophical, and Physical Inquiries into the Foundations of Quantum Mechanics. Plenum Press, New York, 1990.

[64] E. Nelson. Quantum Fluctuations. Princeton University Press, Princeton, N.J., 1985.

[65] R. Omnes. Logical Reformulation of Quantum Mechanics. Journal of Statistical Physics, 53: 893-932, 1988.

[66] W. Pauli. In S. Flügge, editor, Encyclopedia of Physics, Volume 60. Springer, Berlin, Heidelberg, New York, 1958.

[67] A. Peres. Two Simple Proofs of the Kochen-Specker Theorem. Journal of Physics, A24: 175-178, 1991.

[68] E. Prugovecki. Quantum Mechanics in Hilbert Space. Academic Press, New York and London, 1971.

[69] M. Reed and B. Simon. Methods of Modern Mathematical Physics II. Academic Press, New York, 1975.

[70] M. Reed and B. Simon. Methods of Modern Mathematical Physics I. Functional Analysis, revised and enlarged edition. Academic Press, New York, 1980.

[71] F. Riesz and B. Sz.-Nagy. Functional Analysis. F. Ungar, New York, 1955.

[72] E. Schrödinger. Die gegenwärtige Situation in der Quantenmechanik. Naturwissenschaften, 23: 807-812, 1935. English translation by J. D. Trimmer, The present situation in quantum mechanics: a translation of Schrödinger's "cat paradox" paper, Proceedings of the American Philosophical Society, 124: 323-338, 1980. Reprinted in [75].

[73] M. O. Scully, B. G. Englert, and H. Walther. Quantum Optical Tests of Complementarity. Nature, 351: 111-116, 1991.

[74] J. von Neumann. Mathematische Grundlagen der Quantenmechanik. Springer Verlag, Berlin, 1932. English translation by R. T. Beyer, Mathematical Foundations of Quantum Mechanics. Princeton University Press, Princeton, N.J., 1955.

[75] J. A. Wheeler and W. H. Zurek. Quantum Theory and Measurement. Princeton University Press, Princeton, N.J., 1983. 
[76] E. P. Wigner. The Problem of Measurement. American Journal of Physics, 31: 6-15, 1963. Reprinted in [75.

[77] E. P. Wigner. Interpretation of Quantum Mechanics. In [75], 1976.

[78] W. K. Wooters and W. H. Zurek. A Single Quantum cannot be cloned. Nature 299: 802-803, 1982.

[79] W. H. Zurek. Environment-Induced Superselection Rules. Physical Review D 26: 1862-1880, 1982. 